\title{
Propriedade de Bernoulli para bilhares hiperbólicos com fronteiras focalizadoras quase planas.
}

\author{
Rodrigo Manoel Dias Andrade
}

\author{
TESE APRESENTADA \\ $\mathrm{AO}$ \\ Instituto De Matemática e Estatística \\ DA \\ Universidade DE SÃo PAUlo \\ PARA \\ OBTENÇÃO DO TÍTULO \\ $\mathrm{DE}$ \\ DOUTOR EM CIÊNCIAS \\ Programa: Matemática Aplicada \\ Orientador: Prof. Dr. Rodrigo Bissacot \\ Co-orientador: Prof. Dr. Roberto Markarian
}

Durante o desenvolvimento deste trabalho o autor recebeu auxílio financeiro da CAPES

São Paulo, outubro de 2015 


\section{Propriedade de Bernoulli para bilhares hiperbólicos com fronteiras focalizadoras quase planas.}

Esta é a versão original da tese elaborada pelo candidato (Rodrigo Manoel Dias Andrade), tal como submetida à Comissão Julgadora. 


\title{
Propriedade de Bernoulli para bilhares hiperbólicos com fronteiras focalizadoras quase planas.
}

\author{
Esta versão da tese contém as correções e alterações sugeridas \\ pela Comissão Julgadora durante a defesa da versão original do trabalho, \\ realizada em 09/10/2015. Uma cópia da versão original está disponível no \\ Instituto de Matemática e Estatística da Universidade de São Paulo.
}

Comissão Julgadora:

- Prof. Dr. Roberto Markarian (co-orientador) - Universidad de la República

- Prof. Dr. Fábio Tal - IME-USP

- Prof. Dr. Ricardo dos Santos Freire Jr - IME-USP

- Prof. Dr. Rodrigo Bissacot Proença - IME-USP

- Prof ${ }^{\mathrm{a}}$. Dr ${ }^{\mathrm{a}}$. Sonia Pinto de Carvalho - UFMG 


\section{Dedicatória}

À minha mãe Inês, dedico. 


\section{Agradecimentos}

Primeiramente, agradeço a Deus por me conduzir às pessoas certas para a realização deste trabalho. Em particular, ao Rodrigo Bissacot pela sua orientação, dedicação e disponibilidade desde o início do meu doutorado. Ao Roberto Markarian, pelo convite ao Uruguai, onde quase toda parte do trabalho foi desenvolvida lá sob sua supervisão, paciência e hospitalidade.

Aos meus pais Manoel e Inês, por estarem sempre presente pessoalmente ou via skype nos momentos que eu mais precisei. Sem o carinho e incentivo de vocês, esse trabalho nada seria. Família é tudo!

Ao meu irmão e amigo de quarto Plinio, pela convivência e conselhos ao longo desses anos.

À minha irmã pelo amor e apoio incondicional.

À minha namorada Juliana por saber entender o que é a vida de um doutorando.

Ao meu amigo de instituto Edgardo, muito obrigado pelo apoio e amizade.

Um agradecimento especial é devido também aos membros da banca examinadora pelas sugestões e correções do texto.

Ao grupo de Investigação CSIC-Universidad de la República "Sistemas Dinâmicos" pelo apoio financeiro em Montevideo.

À CAPES pelo apoio financeiro no Brasil e Uruguai. 


\section{Resumo}

Dias Andrade, R. M. Propriedade de Bernoulli para bilhares hiperbólicos com fronteiras focalizadoras quase planas. 2015. 120 f. Tese (Doutorado) - Instituto de Matemática e Estatística, Universidade de São Paulo, São Paulo, 2015.

Neste trabalho, mostramos que os bilhares hiperbólicos construídos originalmente por BussolariLenci têm a propriedade de Bernoulli. Tais bilhares não satisfazem as técnicas standard de WojtkowskiMarkarian-Donnay-Bunimovich para bilhares focalizadores hiperbólicos, a qual requer que o diâmetro da mesa do bilhar seja de mesma ordem que o maior raio de curvatura ao longo da componente focalizadora. Nossa prova, utiliza um teorema ergódico local que nos diz que sob certas condições, existe um conjunto de medida total do espaço de fase do bilhar tal que cada ponto desse conjunto possui uma vizinhança contida $(\bmod 0)$ em uma componente Bernoulli da aplicação do bilhar.

Palavras-chave: Bilhares hiperbólicos, Ergodicidade, Propriedade de Bernoulli. 


\section{Abstract}

Dias Andrade, R. M. Bernoulli property for hyperbolic billiards with nearly flat focusing boundaries. 2015. 120 f. Tese (Doutorado) - Instituto de Matemática e Estatística, Universidade de São Paulo, São Paulo, 2015.

In this work, we show that hyperbolic billiards constructed originally by Bussolari-Lenci has the Bernoulli property. These billiards do not satisfy the standard Wojtkowski-Markarian-DonnayBunimovich technique for the hyperbolicity of focusing or mixed billiards in the plane, which requires the diameter of a billiard table to be of the same order as the largest ray of curvature along the focusing boundary. Our proof employs a locally ergodic theorem which says that under a few conditions, there exists a full measure set of the billiard phase space such that each of its points has a neighborhood contained, up to a zero measure set, in one Bernoulli component of the billiard map.

Keywords: Hyperbolic Billiards, Ergodicity, Bernoulli property. 


\section{Sumário}

Lista de Símbolos $\quad$ xi

Lista de Figuras $\quad$ xiii

1 Introdução $\quad 1$

2 Preliminares $\quad 5$

2.1 Domínio do bilhar. . . . . . . . . . . . . . . . . . . . . . 5

2.2 Espaço de fase. . . . . . . . . . . . . . . . . . . . . . 5

2.3 Aplicação do bilhar. . . . . . . . . . . . . . . . . . . . . . . . . 6

2.4 Conjunto de singularidades. . . . . . . . . . . . . . . . . 6

2.5 Campos de cone e tempos de focalização . . . . . . . . . . . . . . . . . . 7

2.6 Formas quadráticas . . . . . . . . . . . . . . . . . . . . . 9

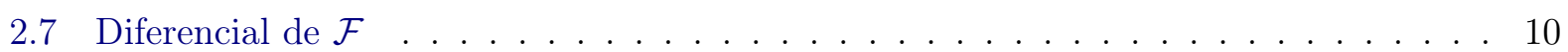

3 Hiperbolicidade. $\quad 13$

4 Ergodicidade Local. $\quad 17$

4.1 Método de Hopf . . . . . . . . . . . . . . . . . . . . . . . . . 17

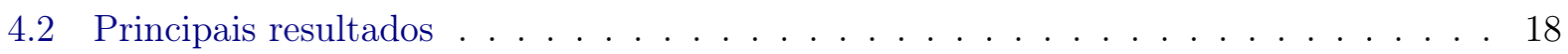

4.3 Ergodicidade local . . . . . . . . . . . . . . . . . . . . . . . . . . . 19

4.3.1 Condição L1 - Regularidade . . . . . . . . . . . . . . . . . . . 20

4.3 .2 Condição L2 - Alinhamento . . . . . . . . . . . . . . . . . . . . 23

4.3 .3 Condição L3 - Sinai-Chernov ansatz . . . . . . . . . . . . . . . . . 24

4.3.4 Condição L4 - Contração . . . . . . . . . . . . . . . . . . . . 33

4.3.5 Campos de Jacobi e semi-normas. . . . . . . . . . . . . . . . . . 33

4.3 .6 Blocos de decomposição. . . . . . . . . . . . . . . . . . . . . . . 36

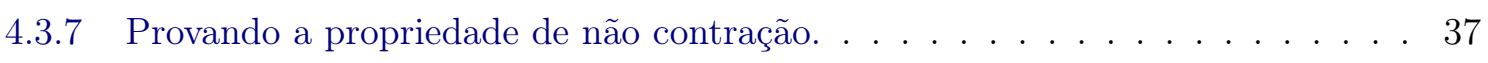

4.3 .8 Conclusão da prova de L4 . . . . . . . . . . . . . . . . . . . . . . . . . . 42

A Teorema da Decomposição Espectral 45

$\begin{array}{ll}\text { Referências Bibliográficas } & 47\end{array}$ 


\section{Lista de Símbolos}

$\begin{array}{ll}\Omega & \text { Domínio do bilhar } \\ \partial \Omega & \text { Fronteira de } \Omega \\ \Gamma_{i} & \text { i-ésima componente de } \partial \Omega \\ \mathcal{K} & \text { Curvatura da componente } \\ \mathcal{M} & \text { Espaço de fase } \\ \mathcal{F} & \text { Mapa do bilhar } \\ \mathcal{R}_{k}^{ \pm} & \text {Conjuntos singulares de } \mathcal{F}^{ \pm k} \\ \{C(x)\}_{x \in U} & \text { Campo de cone } \\ F^{ \pm} & \text {Pontos focais } \\ f^{ \pm} & \text {Distâncias focais relativos aos pontos } F^{ \pm} \\ D_{x} \mathcal{F} & \text { Diferencial de } \mathcal{F} \text { no ponto } x \\ \sigma\left(D_{x} \mathcal{F}^{k}\right) & \text { Taxa de expansão gerada pela ação da derivada } D \mathcal{F}^{k} \\ D_{\beta} & \text { Disco fechado de raio } 1 /|\beta \mathcal{K}| \\ V^{s} & \text { Variedade estável local } \\ V^{u} & \text { Variedade instável local } \\ J(t) & \text { Campo de Jacobi }\end{array}$




\section{Lista de Figuras}

2.1 A reta tangente $t(s)$ e alguns discos $D_{\beta}(s)$. A parte amarela da trajetória é o lugar geométrico dos pontos focais $F^{+}$correspondendo a um certo cone. . . . . . . . . . 11

2.2 Uma representação geométrica da Proposição 1. A figura da esquerda representa as duas primeiras equivalências (componente focalizadora). Os conjuntos amarelo/azul de pontos focais $F^{-}$são mapeados em conjuntos amarelo/azul de pontos focais $F^{+}$. 12

3.1 A mesa de bilhar estudada em [BL08]. . . . . . . . . . . . . . . . . . . . . . 14

3.2 Condição 3 para duas escolhas de $s^{\prime} \ldots \ldots \ldots \ldots \ldots \ldots$

4.1 Uma cadeia de Hopf conectando $x$ e $y \ldots \ldots \ldots \ldots \ldots$

4.2 A principal mesa de bilhar. . . . . . . . . . . . . . . . . . . . . . 18

4.3 Prova da Proposição 2. . . . . . . . . . . . . . . . . . . . . . . . . . . . . . 19

$4.4 \Gamma_{1}$ e $\Gamma_{2}$ no sistema de coordenadas cartesianas $(X, Y) \ldots \ldots \ldots \ldots \ldots \ldots$ 


\section{Capítulo 1}

\section{Introdução}

Um bilhar planar é o sistema mecânico consistindo de um ponto material movendo livremente no interior de um domínio conexo $\Omega \subset \mathbb{R}^{2}$ com fronteira diferencial por partes, e refletindo sobre $\partial \Omega$ de modo que o ângulo de incidência é igual ao ângulo de reflexão.

O estudo das dinâmicas desordenadas no bilhar está diretamente relacionado com a conhecida Hipótese Ergódica de Boltzmann formulada há mais de cem anos pelo físico alemão Ludwing Boltzmann. Tal Hipótese é parte de um modelo mecânico para explicar as propriedades dos gases.

As características específicas dos bilhares aparecem quando o papel da fronteira (curvatura, posição relativa, etc.) é muito mais importante que o da variedade subjacente. Algumas questões interessantes surgem ao estudar os bilhares, como por exemplo: a partícula visita as vizinhanças de todos os pontos da fronteira? Para qualquer ponto inicial, a trajetória de uma partícula é densa no espaço de fase da transformação? O que podemos dizer sobre a ergodicidade desses sistemas? Propriedades como mistura, Kolmogorov (propriedade K) ou Bernoulli implicam em ergodicidade e, portanto, são propriedades fortemente estudadas na teoria de bilhares.

Nesse trabalho, iremos nos concentrar nos bilhares hiperbólicos construídos por Bussolari-Lenci [BL08], isto é, um tipo particular de bilhar cujo mapa possui expoentes de Lyapunov não nulos.

A teoria matemática de bilhares hiperbólicos foi iniciada por Sinai. Em [Sin70], Sinai mostrou que a aplicação do bilhar de um sistema em um toro bidimensional com uma quantidade finita de obstáculos convexos (curvatura positiva) é hiperbólico e K-automorfismo e, portanto, misturador com respeito a sua medida natural finita. Neste, é utilizado o chamado método de Hopf, que consiste na prova de ergodicidade de um sistema a partir da existência de variedades estáveis e instáveis absolutamente contínuas. Gallavotti e Ornstein [GO74] provaram que o bilhar de Sinai também é Bernoulli. Pouco depois, Bunimovich provou que bilhares em alguns domínios com fronteira formada por arcos convexos focalizadores e segmentos de retas, são também hiperbólicos [Bun74, Bun79]. O mais importante exemplo de um bilhar de Bunimovich é o estádio, a região limitada por dois semicírculos conectados por segmentos paralelos. As únicas componentes focalizadoras admissíveis em bilhares de Bunimovich são os arcos de círculo. Essa limitação foi resolvida por Wojtkowski, Markarian, Donnay e Bunimovich. Usando novas técnicas para estabelecer a positividade de expoentes de Lyapunov [Woj86, Mar88], eles provaram independentemente, que vários outros arcos focalizadores podem ser usados para construir bilhares hiperbólicos [Woj86, Mar88, Bun90b, Don91].

As hipóteses da teoria standard de bilhares hiperbólicos de Wojtkowski, Markarian, Donnay e Bunimovich ([CM06], Teo. 9.19) requer que os círculos de semi-curvatura em qualquer ponto de uma componente focalizadora não deve intersectar outras componentes, ou o círculo de semi-curvatura relativo à outras componentes focalizadoras (em [Mar88] tem uma condição similar). Tal condição é requerida para aplicar o chamado mecanismo de desfocagem, que pode ser descrito da seguinte 
forma: deseja-se um feixe divergente de trajetórias para manter-se divergente após cada colisão com a fronteira. Mas em uma porção focalizadora da fronteira, um feixe divergente pode ser rebatido como um feixe convergente. Uma maneira de contornar esse problema, é deixar viajar intacto um feixe convergente por um tempo suficientemente longo até as trajetórias concentrar-se em si e, em seguida, começarem a divergir novamente.

O mecanismo de desfocagem provém da óptica geométrica, porém há formulações analíticas semelhantes na literatura, veja [Mar88, Mar93]. Tal mecanismo, é a extensão mais próxima da original idéia de Sinai de extrair hiperbolicidade das características de fronteiras dispersoras [Sin70].

Entre bilhares de Sinai e Bunimovich, somente casos especiais dos bilhares hiperbólicos restantes foram provados ser ergódicos [LW86, MM13a, Bun90a, CT98, Mag01, Mar93, Szá92]. Nosso principal objetivo é mostrar que os bilhares iniciados por Bussolari-Lenci [BL08], os quais não se aplica a teoria standard de bilhares hiperbólicos de Wojtkowski, Markarian, Donnay e Bunimovich, tem a propriedade de Bernoulli (ou equivalentemente, dizemos 'Bernoulli' ), isto é, isomorfo a um shift de Bernoulli. A propriedade de Bernoulli está no topo da hierarquia ergódica: ela implica Kpropriedade, mixing e ergodicidade. Foi mostrado que vários bilhares, incluindo os bilhares de Sinai e Bunimovich, tem a propriedade de Bernoulli [LW86, Bun90a, CT98, Mag01, Mar93, Szá92, MM03]. Recentemente, Del Magno-Markarian [MM14] provaram que para uma grande classe de bilhares hiperbólicos satisfazendo certas condições, têm a propriedade de Bernoulli. Notavelmente, essa classe inclui os bilhares hiperbólicos construídos por Donnay [Don91]. Para o nosso conhecimento, todos os bilhares hiperbólicos 2-dimensional cumprem tais condições com a exceção de dois casos [BL08, BM99]. Nosso principal objetivo é provar que, os bilhares em [BL08] são localmente ergódicos e têm a propriedade de Bernoulli.

Um passo central na prova da ergodicidade de um bilhar hiperbólico é mostrar que é localmente ergódico, isto é, todas as suas componentes ergódicas de medida positiva são abertas (mod 0). Resultados desse tipo são chamados de Teoremas Ergódicos Local (LETs). Em [MM13a], foi provado que sob certas condições, simplectomorfismos hiperbólicos com singularidades são localmente ergódicos. Esse resultado generaliza um teorema ergódico local de Liverani e Wojtkowski para situações quando o sistema admite um campo de cone invariante contínuo por partes ao invés de contínuo em toda parte [LW86]. Ambos teoremas, são baseados nas idéias e métodos de Sinai. Ele obteve o primeiro teorema ergódico local para bilhares [Sin70], usando uma versão probabilística do argumento devido a E. Hopf para demonstrar a ergodicidade de fluxos geodésicos em superfícies compactas de curvatura negativa [Hop39]. Outros refinamentos do teorema de Sinai foram obtidos em [LW86, CH96, KSS90, SC87].

O teorema ergódico local que iremos aplicar nesse trabalho é o mesmo em [MM13a] que também pode ser encontrado em [MM14] com as devidas modificações, ou seja, iremos verficar todas as suas condições (chamadas de L1-L4). Esse é o principal foco do trabalho. Todas as hipóteses se refere às propriedades da dinâmica próximas ou sobre o conjunto de singularidades $\mathcal{R}_{k}^{+}$(resp. $\mathcal{R}_{k}^{-}$) com $k \in \mathbb{N}$ do espaço de fase consistindo de elementos cujo $k$-ésimo (resp. $-k$-ésimo) iterado atinge um vértice do domínio do bilhar ou possui uma colisão tangencial com a fronteira do domínio. A condição L1 requer que os conjuntos $\mathcal{R}_{k}^{ \pm}$seja uniões finitas de subvariedades $C^{2}$ do espaço de fase do bilhar. Já a condição L2 requer que os espaços tangentes à $\mathcal{R}_{k}^{+}$(resp. $\mathcal{R}_{k}^{-}$) estejam suficientemente próximos à direção estável (resp. instável) do bilhar. A grosso modo, a condição L3 estabelece que as órbitas no futuro (e passado) da maior parte dos elementos de $\mathcal{R}_{1}^{+}$(resp. $\mathcal{R}_{1}^{-}$) tem alguma forma fraca de hiperbolicidade. Essa condição (Ansatz) foi introduzida por Sinai e Chernov [SC87]. Sua prova implica uma análise cuidadosa da taxa de expansão com respeito a uma especial forma quadrática. Finalmente, a condição L4 requer que o tamanho dos vetores na direção instável (resp. estável), medido com a norma gerada pela métrica riemanianna, seja uniformemente limitada superiormente ao longo das órbitas no passado (resp. futuro) do bilhar que terminam em uma vizinhança de certos conjuntos singulares. Essa condição está estritamente relacionada com a 
propriedade de não-contração introduzida em [LW86]. Para a sua prova, iremos estudar separadamente diferentes órbitas do bilhar, e usaremos certas semi-normas definidas em termos de campos de Jacobi transversais. 


\section{Capítulo 2}

\section{Preliminares}

Um sistema de bilhar pode ser descrito ou por um fluxo ou por uma transformação. Nesse trabalho, iremos nos concentrar na transformação do bilhar. Para a relação entre a transformação do bilhar e o fluxo do bilhar, recomendamos ao leitor a referência [CM06]. Nessa seção, iremos definir a transformação do bilhar para um domínio 2-dimensional, veja [CM06]. Observe que há diferenças entre as parametrizações de ângulo e a orientação das distâncias focais nos trabalhos [BL08] e [CM06].

\subsection{Domínio do bilhar.}

Um subconjunto $\Gamma \subset \mathbb{R}^{2}$ é chamado um arco de classe $C^{k}$ com $k \geq 0$ se $\Gamma$ é a imagem de um homeomorfismo (sobre sua imagem) $\gamma:[0,1] \rightarrow \mathbb{R}^{2}$ de classe $C^{k}$. A fronteira de um arco $\Gamma$ é dada por $\partial \Gamma=\gamma(0) \cup \gamma(1)$. Um subconjunto $\Gamma \subset \mathbb{R}^{2}$ é chamado uma curva fechada de classe $C^{k}$ se $\Gamma$ é difeomorfo ao círculo unitário $S^{1}$. Vemos que $\partial \Gamma=\emptyset$ se $\Gamma$ é uma curva fechada.

Seja $\Omega \subset \mathbb{R}^{2}$ um aberto conexo e limitado, a qual chamamos de mesa do bilhar. Assumimos que $\partial \Omega$ é uma união finita de curvas fechadas de classe $C^{0}$ disjuntas. Também assumiremos que $\Gamma:=\partial \Omega=\bigcup_{i=1}^{n} \Gamma_{i}$ com $n>0$ e $\Gamma_{i}$ sendo ou um arco de classe $C^{3}$ ou uma curva fechada de classe $C^{3}$. Denotemos por $L_{i}$ o comprimento da componente $\Gamma_{i}$. Consideremos a parametrização $\gamma_{i}:\left[0, L_{i}\right] \rightarrow \mathbb{R}^{2}$ de $\Gamma_{i}$ pelo comprimento de arco com a propriedade de que o interior de $\Omega$ permaneça do lado esquerdo do vetor tangente $\gamma_{i}^{\prime}(s)$ para $s \in\left[0, L_{i}\right]$. Assumiremos também que:

- a curvatura de $\Gamma_{i}$, calculada com respeito a parametrização $\gamma_{i}$, poderá ser somente estritamente negativa, estritamente positiva ou identicamente igual a zero,

- $\Gamma_{i} \cap \Gamma_{j} \subset \partial \Gamma_{i} \cap \partial \Gamma_{j}$ para $i \neq j$,

- o conjunto de pontos onde $\partial \Omega$ não é $C^{3}$ é exatamente $\bigcup_{i=1}^{n} \partial \Gamma_{i}$.

Os conjuntos $\Gamma_{1}, \ldots, \Gamma_{n}$ são chamados de componentes de $\partial \Omega$. A união de todas as componentes com curvatura positiva (componentes focalizadoras), curvatura negativa (componentes dispersoras) e curvatura zero (componentes flat) são denotadas por $\Gamma^{+}, \Gamma^{-}$e $\Gamma^{0}$, respectivamente. Vemos que uma componente flat é um segmento de reta. Um ponto de $\bigcup_{i=1}^{n} \partial \Gamma_{i}:=\Gamma_{*}$ é chamado de vértice de $\partial \Omega$ e um ponto de $\partial \Omega \backslash \Gamma_{*}:=\tilde{\Gamma}$ é chamado de ponto regular de fronteira.

\subsection{Espaço de fase.}

Para cada $i=1, \ldots, n$ defina $\mathcal{M}_{i}:=\left[0, L_{i}\right] \times[-\pi / 2, \pi / 2]$ com elementos $(0, \alpha)$ e $\left(L_{i}, \alpha\right)$ identificados quando $\Gamma_{i}$ é uma curva fechada. Segue que $\mathcal{M}_{i}$ é um retângulo ou um cilindro. Assim, $\mathcal{M}=\cup_{i} \mathcal{M}_{i}$ é identificado com o retângulo $[0, L] \times[-\pi / 2, \pi / 2]$, onde $L=\sum_{i=1}^{n} L_{i}$ é o comprimento total de $\partial \Omega$. Um elemento $x \in \mathcal{M}$ é, portanto, um par ordenado $(i,(s, \alpha))$, o qual é chamado de um estado ou uma colisão. Definimos $i(x)=i, s(x)=s$ e $\alpha(x)=\alpha$ para $x=(i,(s, \alpha)) \in \mathcal{M}$. 
Para simplificar a notação, identificamos cada $x \in \mathcal{M}$ com o correspondente par $(s, \alpha)$ omitindo (salvo exceções) o índice $i$ da representação $(i,(s, \alpha))$. Quando necessitarmos especificar $i$ escrevemos ' $x \in \mathcal{M}_{i}$ '. Dado $x=(s, \alpha) \in \mathcal{M}_{i}$, defina $q(x)=\gamma_{i}(s) \in \Gamma_{i}$ e $u(x)$ o vetor unitário de $\mathbb{R}^{2}$ apontando para dentro da mesa do bilhar. O conjunto $\mathcal{M}$ é uma variedade diferenciável com fronteira $\partial \mathcal{M}=\bigcup_{i=1}^{n} \partial \mathcal{M}_{i}$. Também, identificaremos o espaço tangente $T_{x} \mathcal{M}$ com $\mathbb{R}^{2}$ tal que se $v \in T_{x} \mathcal{M}$, então $v=(d s, d \alpha)$. Denotemos por $\mathcal{M}^{+}, \mathcal{M}^{-}$e $\mathcal{M}^{0}$ a união de todos os conjuntos $\mathcal{M}_{i}$ com $\Gamma_{i}$ sendo focalizadora, dispersora ou flat, respectivamente.

Equipamos $\mathcal{M}$ com a métrica Riemanniana $g=\left\{g_{x}\right\}_{x \in \mathcal{M}}$ e a forma simplética $\omega=\left\{\omega_{x}\right\}_{x \in \mathcal{M}}$ dada por $g_{x}=d s^{2}+d \alpha^{2}$ e $\omega_{x}=\cos \alpha(x) d s \wedge d \alpha$ para $x \in \mathcal{M}$. A norma gerada por $g$ é denotada por $\|\cdot\|$. A métrica Riemanniana $g$ induz uma distância usual $d$ em cada $\mathcal{M}_{i}$, a qual pode ser estendida em todo $\mathcal{M}$ colocando $d(x, y)=1$ sempre que $x \in \mathcal{M}_{i}$ e $y \in \mathcal{M}_{j}$ com $i \neq j$. Denotemos por $m$ a medida volume gerada por $g$. Assim, $\mu=(2 L)^{-1} \cos \alpha(x) m$ é a medida de probabilidade gerada por $\omega$. Dados $\mathcal{A} \subset \mathcal{M}$ e $\epsilon>0$, seja $\mathcal{A}(\epsilon)=\{y \in \mathcal{M}: d(y, \mathcal{A})<\epsilon\}$ a $\epsilon$-vizinhança de $\mathcal{A}$.

Observamos que existe uma involução natural $\mathcal{I}: \mathcal{M} \rightarrow \mathcal{M}$ dada por $\mathcal{I}(s, \alpha)=(s,-\alpha)$ para cada $(s, \alpha) \in \mathcal{M}_{i}$. Para efeito de notação, iremos escrever $-x$ ao invés de $\mathcal{I}(x)$. Se $B$ é um subconjunto de $\mathcal{M}$, então $-B$ será denotado por $\{-x: x \in B\}$.

\subsection{Aplicação do bilhar.}

Seja $x=(s, \alpha) \in \mathcal{M}_{i}$ e defina $\rho(x)=\{t>0:(q(x), q(x)+t u(x)) \subset \Omega\}$, onde $(q(x), q(x)+t u(x))$ é um segmento aberto de $\mathbb{R}^{2}$ com extremidades $q(x)$ e $q(x)+t u(x)$. Defina também, $\tau(x)=0$ se $\rho(x)=0$, e $\tau(x)=\sup \rho(x)$ caso contrário, e $q_{1}(x)=q(x)+\tau(x) u(x)$. Seja

$$
\mathcal{M}^{\prime}=\left\{x \in \mathcal{M}: q_{1}(x) \text { não pertence a um vertice de } \partial \Omega\right\} .
$$

Se $x \in \mathcal{M}^{\prime}$, então existe um único $i_{1}(x)$ tal que $q_{1}(x)$ pertence ao interior de $\Gamma_{i_{1}(x)}$. Portanto, podemos definir $s_{1}(x)=\gamma_{i_{1}(x)}^{-1}\left(q_{1}(x)\right)$. Considere,

$$
u_{1}(x)=u(x)-2\left\langle\gamma_{i_{1}(x)}^{\prime \prime}\left(s_{1}(x)\right), u(x)\right\rangle \gamma_{i_{1}(x)}^{\prime \prime}\left(s_{1}(x)\right),
$$

onde $\langle\cdot, \cdot\rangle$ denota o produto escalar em $\mathbb{R}^{2}$. Denotemos por $\alpha_{1}(x) \in[-\pi / 2, \pi / 2]$ o ângulo orientado (no sentido horário) entre $u_{1}(x)$ e o vetor normal $\gamma_{i_{1}(x)}^{\prime \prime}\left(s_{1}(x)\right)$ apontando para dentro da mesa.

A aplicação do bilhar para o domínio $\Omega$ é a transformação $\mathcal{F}: \mathcal{M}^{\prime} \rightarrow \mathcal{M}$ dada por

$$
\mathcal{F} x=\left(i_{1}(x),\left(s_{1}(x), \alpha_{1}(x)\right)\right) \text { para } x \in \mathcal{M}^{\prime} .
$$

Notamos que tal transformação não é diferenciável: existem pontos de $\mathcal{M}^{\prime}$, chamados de pontos singulares para os quais $\mathcal{F}$ não é contínua ou duas vezes difrenciável. Daremos agora uma descrição detalhada desses pontos.

\subsection{Conjunto de singularidades.}

Defina,

$$
\begin{aligned}
& A_{1}=\{x \in \mathcal{M}: s \text { está em um vértice de } \partial \Omega\}, \\
& A_{2}=\{x \in \mathcal{M}: \alpha \in\{-\pi / 2, \pi / 2\}\}, \\
& A_{3}=\left\{x \in \mathcal{M} \backslash \partial \mathcal{M}: s_{1} \text { está em um vértice de } \partial \Omega\right\}, \\
& A_{4}=\left\{x \in \mathcal{M} \backslash\left(\partial \mathcal{M} \cup A_{3}\right): \mathcal{F} x \in A_{2}\right\} .
\end{aligned}
$$

Vemos que $\partial \mathcal{M}=A_{1} \cup A_{2}$. Defina também, $\mathcal{S}_{1}^{+}=A_{3} \cup A_{4}$ e $\mathcal{S}_{1}^{-}=-\mathcal{S}_{1}^{+}$, e para cada $k \geq 1$, defina iterativamente $\mathcal{S}_{k+1}^{+}=\mathcal{S}_{k}^{+} \cup \mathcal{F}^{-1} \mathcal{S}_{k}^{+}$e $\mathcal{S}_{k}^{-}=-\mathcal{S}_{k}^{+}$. Finalmente, denotamos por $\mathcal{R}_{k}^{ \pm}=\partial \mathcal{M} \cup \mathcal{S}_{k}^{ \pm}$. 
O conjunto $\mathcal{R}_{k}^{+}$contém os pontos de $\mathcal{M}$ onde $\mathcal{F}^{k}$ ou não está definido ou não é duas vezes diferenciável. Analogamente, o conjunto $\mathcal{R}_{k}^{-}$contém on pontos de $\mathcal{M}$ onde ou $\mathcal{F}^{-k}$ não está definido ou não é duas vezes diferenciável. Os conjuntos $\mathcal{R}_{k}^{+}$e $\mathcal{R}_{k}^{-}$são chamados de conjuntos singulares de $\mathcal{F}^{k}$ e $\mathcal{F}^{-k}$ respectivamente.

Sob as hipóteses acima $\mathcal{F}: \mathcal{M} \backslash \mathcal{R}_{1}^{+} \rightarrow \mathcal{M} \backslash \mathcal{R}_{1}^{-}$é uma aplicação com singularidades, do tipo estudada por Katok e Strelcyn em [KS86]. Nesse mesmo trabalho, é mostrado que $\mathcal{F}$ é um $C^{2}$ difeomorfismo que preserva a forma simplética $\omega$ e a medida de probabilidade $\mu$ (veja [KS86], Corolários 4.1 e 4.4, Part V). Para os bilhares considerados nesse trabalho, mostraremos (veja Subseção 4.3.1) que os conjuntos $\mathcal{R}_{k}^{+}$e $\mathcal{R}_{k}^{-}$são uniões finitas de $\operatorname{arcos}$ de classe $C^{2}$ que se intersectam somente nas extremidades. Assim, com essa propriedade e da invariância de $\mu$ segue que $\mu\left(\mathcal{R}_{k}^{+}\right)=$ $\mu\left(\mathcal{R}_{k}^{-}\right)=0$ para cada $k \geq 1$. Finalmente, observamos que $\mathcal{F}$ é tempo-reversível, isto é, $\mathcal{I} \circ \mathcal{F}=\mathcal{F}^{-1} \circ \mathcal{I}$ em $\mathcal{M} \backslash \mathcal{R}_{1}^{+}$.

Seguindo [MM14], definimos os seguintes conjuntos:

- $\mathcal{R}_{\infty}^{ \pm}:=\bigcup_{k \geq 1} \mathcal{R}_{k}^{ \pm}$,

- $\mathcal{R}:=\mathcal{R}_{\infty}^{-} \cap \mathcal{R}_{\infty}^{+}$,

- $\mathcal{N}^{ \pm}:=\left\{x \in \mathcal{M} \backslash \mathcal{R}_{\infty}^{ \pm}: \exists k>0\right.$ tal que $\left.\mathcal{F}^{( \pm) n} x \in \mathcal{M}^{0} \quad \forall n \geq k\right\}$,

- $\mathcal{N}:=\mathcal{N}^{-} \cap \mathcal{N}^{+}$,

- $\mathcal{N}^{\prime}:=\left(\mathcal{R}_{\infty}^{-} \cap \mathcal{N}^{+}\right) \cup\left(\mathcal{R}_{\infty}^{+} \cap \mathcal{N}^{-}\right)$.

Observação 1. O significado geométrico dos conjuntos anteriores é: $\mathcal{R}_{\infty}^{+}$(resp. $\mathcal{R}_{\infty}^{-}$) é o conjunto de colisões com semi-órbita finita positiva (resp. negativa); $\mathcal{R}$ é o conjunto de colisões com órbita finita $; \mathcal{N}^{+}$(resp. $\mathcal{N}^{-}$) é o conjunto de colisões com semi-órbita positiva (resp. negativa) visitando certamente somente componentes flat de $\partial \Omega ; \mathcal{N}$ é o conjunto de colisões com ambas semi-órbitas visitando certamente somente componentes flat de $\partial \Omega ; \mathcal{N}^{\prime}$ é o conjunto de colisões com uma semiórbita sendo finita e a outra semi-órbita visitando certamente somente componentes flat de $\partial \Omega$.

Ainda entre os resultados de Katok e Strelcyn em [KS86] encontra-se uma versão do Teorema de Oseledec que garante para quase todo $(s, \alpha):=x \in \mathcal{M}$ :

1. Uma decomposição do espaço tangente $T_{x} \mathcal{M}$ em $E_{x}^{+} \oplus E_{x}^{-}$. Esses espaços uni-dimensionais são dinamicamente invariantes no sentido de $(D \mathcal{F})_{x} E_{x}^{ \pm}=E_{\mathcal{F} x}^{ \pm}$, onde $(D \mathcal{F})_{x}$ denota a diferencial de $\mathcal{F}$ em $x$.

2. A existência de expoentes de Lyapunov $\lambda_{ \pm}(x)$, definidos como

$$
\lambda_{ \pm}(x):=\lim _{n \rightarrow+\infty} \frac{1}{n} \log \left\|\left(D \mathcal{F}^{n}\right)_{x} v_{ \pm}\right\|,
$$

com $v_{ \pm} \in E_{x}^{ \pm}$. Como $\mu$ é absolutamente contínua com respeito a medida de Lebesgue em $\mathcal{M}$ então $\lambda_{+}(x)=-\lambda_{-}(x)$. Adotaremos a convenção que $\lambda_{+}(x) \geq 0$.

O sistema dinâmico, por definição, é hiperbólico se $\lambda_{+}(x)>0$ para quase todo $x \in \mathcal{M}$. Se além disso, o sistema é ergódico, então $\lambda_{+}(x)=$ const $:=\lambda_{+}$.

\subsection{Campos de cone e tempos de focalização}

Seguindo [Woj86, LW86], recordaremos as técnicas básicas de campos de cone invariante para a hiperbolicidade de bilhares planares. A invariância do campo de cone é também uma ferramenta muito útil nas provas da ergodicidade dos bilhares estudadas nesse trabalho. Restringiremos as presentes definições e propriedades dos campos de cone ao setting bidimensional.

Seja $V$ um espaço vetorial bidimensional. Dados dois vetores linearmente independentes $X_{1}$ e $X_{2}$ de $V$, dizemos que o conjunto $C\left(X_{1}, X_{2}\right):=\left\{a_{1} X_{1}+a_{2} X_{2}: a_{1} a_{2} \geq 0\right\} \subset V$ é o cone gerado por 
$X_{1}$ e $X_{2}$. Também definimos intC $=\left\{a_{1} X_{1}+a_{2} X_{2}: a_{1} a_{2}>0\right\} \cup\{0\}$ e $C^{\prime}\left(X_{1}, X_{2}\right)=C\left(X_{1},-X_{2}\right)$, chamados interior de $C\left(X_{1}, X_{2}\right)$ e cone complementar de $C\left(X_{1}, X_{2}\right)$, respectivamente.

Agora, seja $U$ um conjunto aberto de $\mathcal{M}$, e suponhamos que $X_{1}$ e $X_{2}$ são dois campos de vetores mensuráveis em $U$ tais que $X_{1}(x)$ e $X_{2}(x)$ são linearmente independentes para todo $x \in U$. Um campo de cone $C$ em $U$, denotado por $(U, C)$, é uma família de cones $\{C(x)\}_{x \in U}$ dado por $C(x)=C\left(X_{1}(x), X_{2}(x)\right) \subset T_{x} \mathcal{M}$ para cada $x \in U$. Um campo de cone $(U, C)$ é chamado contínuo se o campo de vetores $X_{1}$ e $X_{2}$ são contínuos em $U$.

Um campo de cone $(U, C)$ é dito:

- invariante (resp. estritamente invariante) se $D_{x} \mathcal{F} C(x) \subset C\left(\mathcal{F}^{k} x\right)\left(\right.$ resp. $\left.D_{x} \mathcal{F} C(x) \subset \operatorname{intC}\left(\mathcal{F}^{k} x\right)\right)$, para $x \in U$ e $\mathcal{F}^{k} x \in U \operatorname{com} k>0$.

- certamente estritamente invariante se é invariante, e para quase todo $x \in U$, existe $k(x)>0$ tal que $\mathcal{F}^{k(x)} x \in U$ e $D_{x} \mathcal{F}^{k(x)} C(x) \subset \operatorname{int} C\left(\mathcal{F}^{k(x)}\right)$.

O próximo teorema foi provado em [Woj85].

Teorema 1. Dada uma aplicação do bilhar $\mathcal{F}$ como descrita acima, se existe um campo de cone mensurável, definido para $\mu$-quase todo ponto $x \in \mathcal{M}$, que é certamente estritamente invariante, então o expoente de Lyapunov $\lambda_{+}(x)$ é positivo para $\mu$-quase todo $x \in \mathcal{M}$.

Notamos que para verificar a invariância dos cones no sentido acima, não precisamos conhecer $D_{x} \mathcal{F}$ em si, mas somente como a derivada age sobre as retas em $T_{x} \mathcal{M}$ passando pela origem, isto é, no espaço projetivo real 1-dimensional.

Aqui daremos algumas ideias de como Wojtkowski em [Woj86] mostrou que o expoente de Lyapunov $\lambda_{+}$pode ser de fato estimado inferiormente em termos projetivos.

Assuma que para ( $\mu$-q.t.p.) cada $x \in \mathcal{M}$ escolhamos uma coordenada projetiva $t,-\infty \leq t \leq$ $+\infty$, no espaço projetivo 1-dimensional de retas em $T_{x} \mathcal{M}$ (isto é, para coordenadas euclidianas apropriadas $\left(u_{1}, u_{2}\right)$ em $T_{x} \mathcal{M} t,-\infty<t<+\infty$ corresponde à reta $u_{2}=t u_{1}$ e $t= \pm \infty$ corresponde à reta $u_{1}=0$, ou seja, $t$ é a tangente de ângulo de $u_{2}=0$ com a reta em $\left.T_{x} \mathcal{M}\right)$. As retas no cone $C(x)$ formam um intervalo fechado $I(x)$ no espaço projetivo, $I(x)=\{t \mid l(x) \leq t \leq r(x)\}$, onde $l(x)<r(x)$. Se o campo de cone $C(x), x \in \mathcal{M}$, é invariante por $\mathcal{F}$, então as retas no cone $D_{x} \mathcal{F} C(x)$ formam um sub-intervalo $I_{1}(x)$ de $I(\mathcal{F}(x))$. Se $I_{1}(x)=\left\{t \mid l_{1}(x) \leq t \leq r_{1}(x)\right\}, l_{1}(x)<r_{1}(x)$, então temos $\mu$-q.t.p. $l(\mathcal{F}(x)) \leq l_{1}(x)<r_{1}(x) \leq r(\mathcal{F}(x))$. Seja $N=\left\{x \in \mathcal{M} \mid l(\mathcal{F}(x))<l_{1}(x)\right.$ e $r_{1}(x)<$ $r(\mathcal{F}(x))\}$, e para $x \in N$ seja $\zeta(x)$ o cross ratio $\left[l_{1}(x), r_{1}(x), l(\mathcal{F}(x)), r(\mathcal{F}(x))\right]$ dos quatro pontos, isto é,

$$
\zeta(x)=\frac{r(\mathcal{F}(x))-l_{1}(x)}{r(\mathcal{F}(x))-r_{1}(x)} \cdot \frac{r_{1}(x)-l(\mathcal{F}(x))}{l_{1}(x)-l(\mathcal{F}(x))} .
$$

Vemos que $\zeta(x)>1$ para $x \in N . \zeta(x)$ não depende da escolha da coordenada projetiva $t$ mas somente dos cones $C(\mathcal{F}(x))$ e $D_{x} \mathcal{F} C(x)$. Heuristicamente, $\zeta(x)$ representa o quociente das diferenças entre os limitantes (superior e inferior) dos campos de cone $C(\mathcal{F} x)$ e $D_{x} \mathcal{F} C(x)$.

Teorema 2. (Wojtkowski, [Woj86]) Se um campo de cone mensurável $C(x), x \in \mathcal{M}$, é invariante sobre $\mathcal{F}$, então

$$
\int_{\mathcal{M}} \lambda_{+} d \mu \geq \int_{N} \ln \frac{\sqrt{\zeta}+1}{\sqrt{\zeta-1}} d \mu
$$

onde $N$ e $\zeta$ são definidos acima.

Em [Woj86], Wojtkowski reduz a invariância de um campo de cone à um problema de óptica geométrica com relação ao comportamento de uma família (feixe) de trajetórias próximas. Apresentaremos agora os conceitos de tempos de focalização de uma família infinitesimal de trajetórias. Isso permite obter uma descrição intuitiva da ação da derivada da aplicação do bilhar na reta projetiva. 
Para fins de notação, dada uma subvariedade $B$ de $\mathcal{M}$ e um ponto $x \in B$, denotamos $T_{x}^{*} B$ para $T_{x} B \backslash\{0\}$.

Seja $x \in \mathcal{M}$. Para um vetor tangente $v \in T_{x}^{*} \mathcal{M}$ no espaço de fase é naturalmente associado uma curva diferenciável $\varphi:(-\epsilon, \epsilon) \longrightarrow \mathcal{M}$ tal que $\varphi(0)=x$ e $\varphi^{\prime}(0)=v$. Por construção, $\sigma \mapsto \varphi(\sigma)$ é unicamente determinado na aproximação linear em torno de 0 . Usando a representação de $\mathcal{M}$ como um subconjunto de $\Omega \times S^{1}$, e a notação $\varphi(\sigma)=(q(\sigma), u(\sigma)) \in \Omega \times S^{1}$, construimos a família de retas, $l^{+}(\sigma):=\{q(\sigma)+r u(\sigma) \mid r \in \mathbb{R}\}$. Também denotando por $u^{-}(\sigma)$ o vetor pré-colisional de $u(\sigma)$ apontando para fora em $q(\sigma) \in \Gamma$, definimos $l^{-}(\sigma):=\left\{q(\sigma)+r u^{-}(\sigma) \mid r \in \mathbb{R}\right\}$. Na primeira aproximação, isto é, quando $\epsilon \rightarrow 0^{+}$, o feixe infinitesimal de retas focaliza em um ponto, isso significa que todas as retas, até os ajustes de ordem $\epsilon$ em $(q(\sigma), u(\sigma))$ tem uma interseção comum. Consideramos também o caso onde a interseção comum é no infinito. Esse ponto focal é claramente uma função de $v$ somente: tal ponto é denotado por $F^{+}(v)$ para a família $\left\{l^{+}(\sigma)\right\}$ e $F^{-}(v)$ para a família $\left\{l^{-}(\sigma)\right\}$. Denotamos por $f^{ \pm}(v)$ as distâncias com sinal, ao longo de $l^{ \pm}(0)$, entre $F^{ \pm}(v)$ e $q_{0}=q(0)\left(l^{ \pm}(\sigma)\right.$ possui orientação induzida pelo parâmetro $r \in \mathbb{R}$, isto é, fora para $l^{-}(\sigma)$ e dentro para $l^{+}(\sigma)$, relativo a $\Omega$ ). Daqui para frente, omitiremos a dependência de $v$ de todas as notações sempre que não houver ambiguidade. Indicando por $(d s, d \alpha)$ as componentes de $0 \neq v \in T \mathcal{M}_{\left(s_{0}, \alpha_{0}\right)}$ na base natural $\{\partial / \partial s, \partial / \partial \alpha\}$, temos

$$
f^{ \pm}=\left\{\begin{array}{cc}
\frac{\cos \alpha_{0}}{ \pm \mathcal{K}\left(s_{0}\right)-\frac{d \alpha}{d s}}, & d s \neq 0 \\
0, & d s=0
\end{array}\right.
$$

Aqui $\mathcal{K}(s)$ denota a curvatura de $\Gamma$ no ponto de coordenada $s$, em que é positiva em pontos de componente focalizadora e negativa em pontos de componente dispersora. A fórmula em (2.2) pode ser encontrada em [Woj86].

Observação 2. Um cone bidimensional $C$ pode ser naturalmente identificado com um intervalo fechado I do espaço projetivo $\mathbb{P}(V)$. Da mesma forma, intC e $C^{\prime}$ são então identificados com o interior de $I$ e o intervalo fechado $\mathbb{P}(V) \backslash$ intI, respectivamente. Seja $(U, C)$ um campo de cone com $U \subset \mathcal{M} \backslash A_{2}$. Para $x \in U \backslash A_{2}$, como os tempos de focalização $f^{+}$e $f^{-}$são transformações projetivas de $T_{x} \mathcal{M}$, existem dois intervalos fechados $I_{+}(x)$ e $I_{-}(x)$ de $\mathbb{R} \cup\{\infty\}$ tais que o cone $C(x) \in T_{x} \mathcal{M}$ pode ser convenientemente descrito de duas maneiras diferentes:

$$
C(x)=\left\{v \in T_{x}^{*} \mathcal{M}: f^{+}(v) \in I_{+}(x)\right\} \cup\{0\},
$$

ou

$$
C(x)=\left\{v \in T_{x}^{*} \mathcal{M}: f^{-}(v) \in I_{-}(x)\right\} \cup\{0\} .
$$

O próxima lema é conhecido na óptica como a equação do espelho [CM06, Woj86].

Lema 1. Para um feixe infinitesimal de trajetórias colidindo em torno do ponto $s \in \Gamma$ com ângulos de reflexão em torno de $\alpha$, tem-se

$$
-\frac{1}{f^{-}}+\frac{1}{f^{+}}=\frac{2 \mathcal{K}(s)}{\cos \alpha}
$$

\subsection{Formas quadráticas}

Considere um campo de cone $(U, C)$ gerado pelos campos de vetores $X_{1}$ e $X_{2}$. A forma quadrática $Q=\left\{Q_{x}\right\}_{x \in U}$ associada à $(U, C)$ é definida por

$$
Q_{x}(v)=\omega_{x}\left(v_{1}, v_{2}\right) \quad \text { para } x \in U,
$$

onde $v_{1}$ e $v_{2}$ são vetores dos subespaços gerados por $X_{1}(x)$ e $X_{2}(x)$, respectivamente, tal que $v=v_{1}+v_{2}$.

A forma quadrática $Q$ é chamada: 
- monótona (resp. estritamente monótona) se $Q_{\mathcal{F}^{k} x}\left(D_{x} \mathcal{F}^{k} v\right) \geq Q_{x}(v)\left(\right.$ resp. $Q_{\mathcal{F}^{k} x}\left(D_{x} \mathcal{F}^{k} v\right)>$ $\left.Q_{x}(v)\right)$ para todo $v \in T_{x}^{*} \mathcal{M}$ sempre que $x \in U$ e $\mathcal{F}^{k} x \in U$ com $k>0$.

- certamente estritamente monótona se é monótona, e para quase todo $x \in U$, existe um inteiro $k(x)>0$ tal que $Q_{\mathcal{F} k(x) x}\left(D_{x} \mathcal{F}^{k(x)} v\right)>Q_{x}(v)$ para todo $v \in T_{x}^{*} \mathcal{M}$.

Seguindo [LW86], definiremos agora a taxa de expansão gerada pela ação de $D \mathcal{F}^{k} \operatorname{com} k>0$ nos vetores em $(U, C)$ com respeito a forma $Q$.

Definição 1. Seja $C$ um campo de cone invariante definido em $U$, e seja $Q$ a forma quadrática associada. Para cada $x \in U$ e $k>0$ tal que $\mathcal{F}^{k} x \in U$, seja

$$
\sigma_{C}\left(D_{x} \mathcal{F}^{k}\right)=\inf _{v \in \operatorname{int} C(x)} \sqrt{\frac{Q_{\mathcal{F}^{k} x}\left(D_{x} \mathcal{F}^{k} v\right)}{Q_{x}(v)}}
$$

$e$

$$
\sigma_{C}^{*}\left(D_{x} \mathcal{F}^{k}\right)=\inf _{v \in \operatorname{int} C(x)} \frac{\sqrt{Q_{\mathcal{F}^{k} x}\left(D_{x} \mathcal{F}^{k} v\right)}}{\|v\|} .
$$

Para $k<0$, definimos $\sigma_{C}$ e $\sigma_{C}^{*}$ substituindo o campo de cone na definição acima com o seu campo de cone complementar $C^{\prime}(x)$ e $-Q$, respectivamente.

- O campo de cone $(U, C)$ é invariante (resp. estrit. invariante) $\Longleftrightarrow$ a forma quadrática $Q$ é monótona (resp. estrit. monótona),

- $(U, C)$ é certamente estritamente monótona $\Longleftrightarrow Q$ é certamente estritamente monótona.

Além disso, $D_{x} \mathcal{F}^{k} C(x) \subset C\left(\mathcal{F}^{k} x\right)\left(\right.$ resp. $D_{x} \mathcal{F}^{k} C(x) \subset \operatorname{int} C\left(\mathcal{F}^{k} x\right)$ ) para $x \in U$ tal que $\mathcal{F}^{k} x \in U$ com $k>0$ é equivalente à $\sigma_{C}\left(D_{x} \mathcal{F}\right) \geq 1\left(\right.$ resp. $\left.\sigma_{C}\left(D_{x} \mathcal{F}\right)>1\right)$. Veja [LW86] para uma prova detalhada dessas propriedades.

Das definições de invariância estrita de um campo de cone, $\sigma_{C}$ e $\sigma_{C}^{*}$, pode-se deduzir que se $k_{1}, k_{2}, n \in \mathbb{Z}$ com $k_{1} k_{2} \geq 0$, então

$$
\sigma_{C}\left(D_{x} \mathcal{F}^{n}\right)>1 \Longrightarrow \sigma_{C}^{*}\left(D_{x} \mathcal{F}^{n}\right)>0
$$

e

$$
\sigma_{C}^{*}\left(D_{x} \mathcal{F}^{k_{1}+k_{2}}\right) \geq \sigma_{C}^{*}\left(D_{x} \mathcal{F}^{k_{1}}\right) \cdot \sigma_{C}\left(D_{\mathcal{F}^{k_{1}} x} \mathcal{F}^{k_{2}}\right) .
$$

Definição 2. Seja $n(x)=\sup \left\{j \geq 0: x \in \mathcal{M} \backslash \mathcal{R}_{j}^{+} \quad\right.$ e $\left.x, \mathcal{F} x, \ldots, \mathcal{F}^{j} x \in \mathcal{M}_{i(x)}\right\}$ para $x \in \mathcal{M} \backslash A_{2}$, onde $\mathcal{R}_{0}^{+}:=\emptyset$. Se $x \in \mathcal{M}^{-}$, observamos que $n(x)=0$.

O número $n(x)$ é sempre finito, e representa o número de colisões consecutivas da trajetória de $x$ com a componente $\Gamma_{i(x)} \subset \Gamma^{+}$antes de deixá-la.

Definição 3. Dada $\Gamma_{i} \subset \Gamma^{+}$, defina $E_{i}=\left\{x \in \mathcal{M}_{i} \backslash A_{2}: n(-x)=0\right\}$. Também, seja $E^{+}$a união de todos os $E_{i}^{\prime}$ s.

Vemos que, se $x \in E_{i}$ então $\left\{x, \mathcal{F} x, \ldots, \mathcal{F}^{n(x)} x\right\}$ é a maior sequência de colisões consecutivas com $\Gamma_{i}$; qualquer outra colisão que antecede $x$ deverá pertencer à $\mathcal{M}_{j} \operatorname{com} j \neq i$.

\subsection{Diferencial de $\mathcal{F}$}

Identificaremos a diferencial de $D_{x} \mathcal{F}$ com a diferencial da transformação $(s, \alpha) \mapsto\left(s_{1}, \alpha_{1}\right)$. Para cada $x \in \mathcal{M} \backslash \mathcal{R}_{1}^{+}$a matriz da diferencial $D_{x} \mathcal{F}$ é dada por (veja [CM06]):

$$
D_{x} \mathcal{F}=-\frac{1}{\cos \alpha_{1}}\left(\begin{array}{cc}
-\tau \mathcal{K}+\cos \alpha & \tau \\
\tau \mathcal{K} \mathcal{K}_{1}-\mathcal{K} \cos \alpha_{1}-\mathcal{K}_{1} \cos \alpha & -\tau \mathcal{K}_{1}+\cos \alpha_{1}
\end{array}\right)
$$


Apresentaremos agora, uma descrição visual do cone $C(x)=C(s, \alpha)$ no plano de configuração contendo $\Omega$ que foi construído em [BL08]. Para $s \in \partial \Omega$ e $\beta>0$, denotamos por $D_{\beta}$ o disco fechado de raio $1 /|\beta \mathcal{K}(s)|$ tangente à $\partial \Omega$ em $s$ sobre o lado externo de $\Omega$. Considere também, dois semiplanos fechados delimitados por $t(s)$, a reta tangente à $\partial \Omega$ em $s$ : seja $D_{0+}(s)$ o semiplano interno, relativo à $\Omega$, e $D_{0-}(s)$ o externo. Veja Figura 2.1. O interior de $D_{\beta}$ é indicado com $D_{\beta}^{\circ}(s)$.

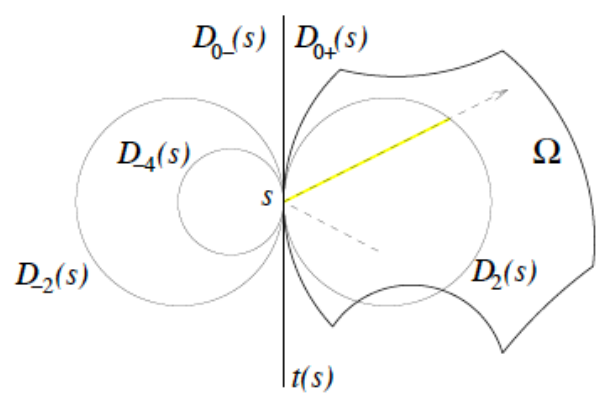

Figura 2.1: A reta tangente $t(s)$ e alguns discos $D_{\beta}(s)$. A parte amarela da trajetória é o lugar geométrico dos pontos focais $F^{+}$correspondendo a um certo cone.

Os próximos resultados bem como suas respectivas provas podem ser encontrados em [BL08].

Lema 2. Dado um campo de cone $C(s, \alpha)$ como na Subseção 2.5, $v \in C(s, \alpha)$ corresponde à $F^{+}(v) \in l^{+}(0) \cap D$, onde $D \subset \mathbb{R}^{2}$ é um dos seguintes conjuntos:

(a) $D=D_{\beta_{1}}(s)$, para algum $\beta_{1} \in \mathbb{R}$;

(b) $D=D_{\beta_{1}}(s) \backslash D_{\beta_{2}}^{\circ}(s)$, para alguns $\beta_{1}, \beta_{2} \in \mathbb{R}$ com $\left|\beta_{1}\right|<\left|\beta_{2}\right|$;

(c) $D=D_{\beta_{1}}(s) \cup D_{\beta_{2}}(s)$, para alguns $\beta_{1}, \beta_{2}$ com $\beta_{1} \geq 0$ e $\beta_{2} \leq 0$;

(d) $D=\mathbb{R}^{2} \backslash\left(D_{\beta_{1}}^{\circ}(s) \cup D_{\beta_{2}}^{\circ}(s) \cup\{s\}\right)$, para alguns $\beta_{1}, \beta_{2}$ com $\beta_{1} \geq 0$ e $\beta_{2} \leq 0$.

Além disso, $F^{+}(v) \in \partial D_{\beta}(s) \backslash\{s\} \Longleftrightarrow f^{+}(v)=\frac{2 \cos \alpha}{\beta|\mathcal{K}(s)|}$.

Veremos que descrevendo os cones em termos dos discos $D_{\beta}(s)$ será eliminada a dependência sobre $\alpha$ na equação do espelho no Lema 1.

Lema 3. Para um feixe infinitesimal de trajetórias colidindo em torno de $s \in \partial \Omega, F^{-} \in \partial D_{\beta}(s)$ se, e somente, se $F^{+} \in \partial D_{\beta^{\prime}}(s)$, onde $\beta^{\prime}=4 \operatorname{sgn}(\mathcal{K}(s))-\beta$ (em que $F^{ \pm} \in \partial D_{0 \pm}$ significa $F^{ \pm} \in\{s, \infty\}$ ).

Com as ferramentas da Subseção 2.5, o problema da invariância do campo de cone ao longo de uma dada trajetória pode ser reduzida ao estudo de pontos focais de perturbações a um parâmetro dessa trajetória.

A proposição a seguir será útil em nossa prova.

Proposição 1. Para um feixe infinitesimal de trajetórias colidindo em torno de s, temos: Se $s \in$ $\mathcal{M}^{+}$, então:

- $F^{\mp} \in D_{4}(s) \Longleftrightarrow F^{ \pm} \in D_{0-}(s)$;

- $F^{\mp} \in D_{2}(s) \backslash D_{4}^{\circ}(s) \Longleftrightarrow F^{ \pm} \in D_{0+}(s) \backslash D_{2}^{\circ}(s)$.

Se $s \in \mathcal{M}_{i}^{-}$para algum $i$, então:

- $F^{\mp} \in D_{-4}(s) \Longleftrightarrow F^{ \pm} \in D_{0+}(s)$;

- $F^{\mp} \in D_{-2}(s) \backslash D_{-4}^{\circ}(s) \Longleftrightarrow F^{ \pm} \in D_{0-}(s) \backslash D_{-2}^{\circ}(s)$.

Para o interior de tais cones, segue com equivalências análogas. A situação é ilustrada na Figura 2.2. 

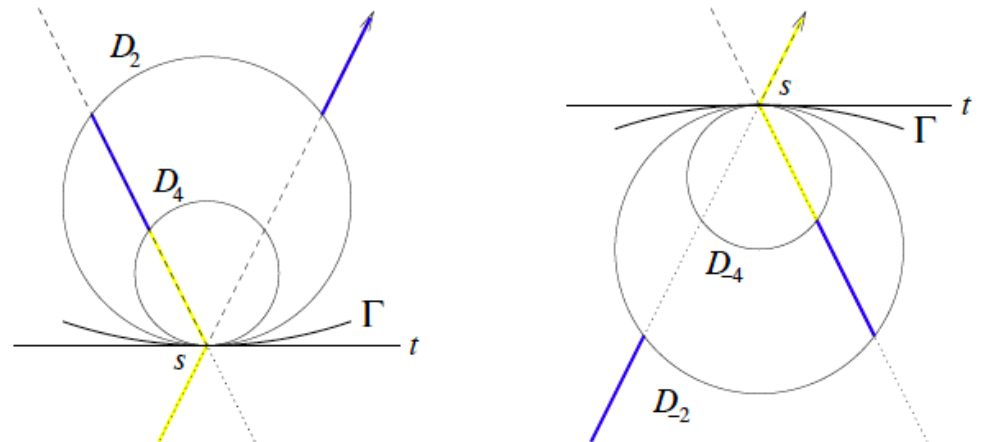

Figura 2.2: Uma representação geométrica da Proposição 1. A figura da esquerda representa as duas primeiras equivalências (componente focalizadora). Os conjuntos amarelo/azul de pontos focais $F^{-}$são mapeados em conjuntos amarelo/azul de pontos focais $F^{+}$. 


\section{Capítulo 3}

\section{Hiperbolicidade.}

Nesse capítulo, iremos descrever os bilhares estudados em [BL08].

Tome um quadrado unitário e substitua três de seus lados por arcos circulares de curvatura $\mathcal{K}_{d} \in(-\sqrt{2}, 0)$ tendo suas extremidades nos vértices do quadrado. A condição $\left|\mathcal{K}_{d}\right|<\sqrt{2}$ assegura que cada par de arcos adjacentes intersecta somente na extremidade comum. Agora, substitua o quarto lado em um arco circular focalizador de curvatura $\mathcal{K}_{f} \ll 1$ e cole duas faixas formadas por regiões poligonais com ângulos interiores racionais como mostra numa versão simplificada a Figura 3.1. Denotamos a altura e comprimento dessas faixas por $h$ e $l$, respectivamente.

Para a prova da hiperbolicidade desse sistema de bilhar, Bussolari-Lenci em [BL08], não usam exatamente a seção de Poincaré que foi introduzida na Seção 2.1, mas uma seção similar que negligencia colisões nas componentes flat's. Esse é um procedimento padrão na teoria de bilhares hiperbólicos em que as colisões contra uma componente flat não alteram as características hiperbólicas de um feixe de trajetórias. Um maneira de ver isso é fazer o desdobramento do bilhar ao longo de uma dada trajetória: após o ponto material colidir com uma componente flat, deixamos sua trajetória mover em linha reta, onde é feita a reflexão da mesa em torno desse lado flat; além desse movimento rígido da mesa do bilhar, nada se altera para a trajetória ou qualquer de suas perturbações infinitesimais.

Dessa forma, denotamos $\tilde{\Gamma}:=\Gamma^{+} \cup \Gamma^{-}$. Com o usual abuso de notação, um ponto $q \in \Gamma$ será identificado com o seu comprimento de arco $s$ e definimos $\mathcal{M}=\tilde{\Gamma} \times[-\pi / 2, \pi / 2]$ cujos elementos chamamos de $(s, \alpha)$ ou $x$. Vemos que $\mathcal{M}$ é uma seção transversal global para o fluxo do bilhar, ou seja, para quase todas as trajetórias do bilhar tem colisões com $\tilde{\Gamma}$. Isso se deve ao fato de um resultado conhecido da teoria de bilhares poligonais (veja [BKM78], Seção 5, pág. 539): seja $P$ a união fechada das duas faixas com $R$, onde $R$ é o quadrilátero unindo as duas extremidades abertas das faixas. $P$ é um polígono racional, o que significa, que todos os seus ângulos são múltiplos racional de $\pi$. Em um bilhar poligonal racional, quase toda trajetória do fluxo não singular no espaço de configuração (isto é, o conjunto $\{q(t)\}_{t \in \mathbb{R}}$ desde que não contenha vértice de $P$ ) com vetor velocidade inicial $u$, é denso em $P$ [BKM78]. Isso implica que para quase toda condição incial $(q, u)$, com $q \in P$, a trajetória do bilhar em $P$, colide com a fronteira de $R$, que significa que a verdadeira trajetória do bilhar, relativo a mesa $\Omega$, colide com $\tilde{\Gamma}$. Esse resultado implica imediatamente que $\mu\left(\mathcal{N}^{ \pm}\right)=0$.

Assim, como $\mathcal{M}$ é uma seção transversal global para o fluxo, consideramos $\mathcal{F}$ definida para quase todo $x \in \mathcal{M}$ sua aplicação de primeiro retorno.

Em [BL08], foi mostrada a hiperbolicidade de um sistema de bilhar tendo como $\Omega$ a região explicada acima, definindo um campo de cone que explora o fato que a componente focalizadora é quase plana, e assim quase sempre age como uma fronteira semidispersora.

As constantes geométricas $l, \mathcal{K}_{f}$ e $\mathcal{K}_{d}$ são escolhidas via o seguinte procedimento. Inicialmente, fixe valores arbitrários de $h$ e $\mathcal{K}_{d}$. Então $\mathcal{K}_{f}$ é determinado por uma condição geométrica que depende 


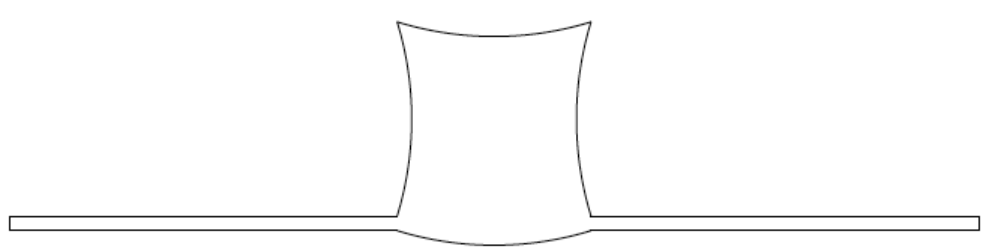

Figura 3.1: A mesa de bilhar estudada em [BL08].
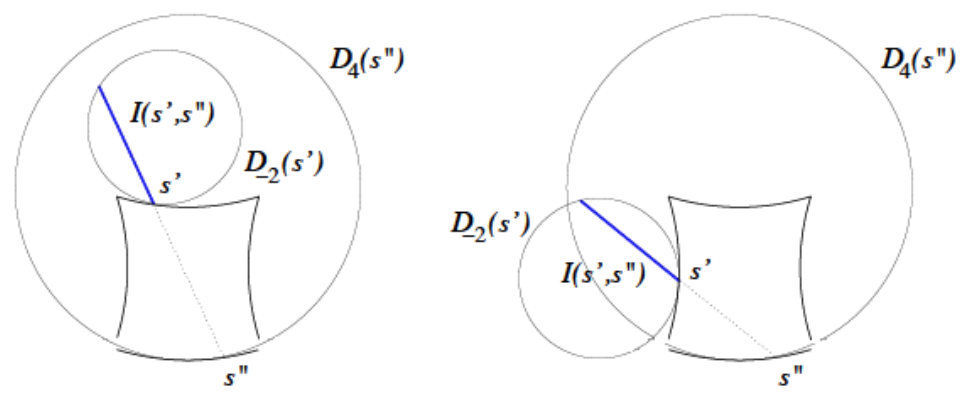

Figura 3.2: Condição 3 para duas escolhas de $s^{\prime}$.

de $\mathcal{K}_{d}$ e $h$ que descrevemos aqui com ajuda da Figura 3.2. Para $s^{\prime} \in \mathcal{M}^{-}$e $s^{\prime \prime} \in \mathcal{M}^{+}$, considere a reta passando por $s^{\prime}$ e $s^{\prime \prime}$, e seja $I\left(s^{\prime}, s^{\prime \prime}\right)$ sua interseção com o disco $D_{-2}\left(s^{\prime}\right)$. A curvatura $\mathcal{K}_{f}$ deve ser pequena de modo que

$$
\forall s^{\prime} \in \mathcal{M}^{-}, \forall s^{\prime \prime} \in \mathcal{M}^{+}, I\left(s^{\prime}, s^{\prime \prime}\right) \subset D_{4}\left(s^{\prime \prime}\right) .
$$

Finalmente, $l$ é escolhido tal que

$$
l \geq 1 / \mathcal{K}_{f} .
$$

Observação 3. Evidentemente, as hipóteses da teoria standard de Wojtkowski, Markarian, Donnay e Bunimovich (veja [CM06], Teorema 9.19) são violadas na condição (3.1), uma vez que $D_{4}\left(s^{\prime \prime}\right)$ contém grandes porções de $\mathcal{M}^{-}$para todo $s \in \mathcal{M}^{+}$.

Para qualquer $x=(s, \alpha) \in \mathcal{M}$ e $n \in \mathbb{Z}$, denotemos $x_{n}:=\left(s_{n}, \alpha_{n}\right):=\mathcal{F}^{n} x$ e seja $\tau_{n}$ o comprimento (equivalentemente ao tempo) entre as colisões em $s_{n}$ e $s_{n+1}$ (vemos que pode haver um número arbitrário de colisões com $\Gamma^{0}$ entre $s_{n}$ e $\left.s_{n+1}\right)$. Também, seja $\mathcal{K}_{n}=\mathcal{K}\left(s_{n}\right)$ a curvatura de $\Gamma$ em $s_{n}$. Analogamente, dado $v \in T_{x} \mathcal{M}$, denotemos por $v_{n}:=\left(D \mathcal{F}^{n}\right)_{x} v$. O feixe infinitesimal de trajetórias determinado por $v_{n}$ (e assim por $v$ ) em torno de $\left(s_{n}, \alpha_{n}\right)$ irão ter focos pré- e pós-colisional denotados, respectivamente, por $F_{n}^{-}:=F^{-}\left(v_{n}\right)$ e $F_{n}^{+}:=F^{+}\left(v_{n}\right)$. As correspondentes distâncias com sinal ao longo das retas pré- e pós-colisional são indicadas com $f_{n}^{-}$e $f_{n}^{+}$.

Vemos que:

$$
\begin{gathered}
F_{n}^{-}=F_{n-1}^{+}, \\
f_{n}^{-}=-\left(\tau_{n-1}-f_{n-1}^{+}\right) .
\end{gathered}
$$

Ainda seguindo [BL08], definimos os três seguintes cones em $T_{x} \mathcal{M}$ para cada $x \in \mathcal{M}$ :

- $C_{0}(x)$ é o conjunto de todos os vetores tangentes cuja correspondente família de retas focaliza na aproximação linear dentro de $D_{-2}(s)$. Usando a distância focal $f^{+}$,

$$
C_{0}(x):=\left\{v \in T_{x} \mathcal{M}:-\frac{\cos \alpha}{|\mathcal{K}|} \leq f^{+}(v) \leq 0\right\} .
$$

- $C_{1}(x)$ é o conjunto de todos os vetores tangentes cuja correspondente família de retas focaliza na aproximação linear dentro de $D_{0-}(s)$, isto é, todas as famílias divergentes de retas. Em termos projetivos,

$$
C_{1}(x):=\left\{v \in T_{x} \mathcal{M}:-\infty<f^{+}(v) \leq 0\right\} .
$$


- $C_{2}(x)$ é o conjunto de todos os vetores tangentes cuja correspondente família de retas focaliza na aproximação linear dentro de $D_{2}(s) \backslash D_{4}^{\circ}(s)$, isto é,

$$
C_{2}(x):=\left\{v \in T_{x} \mathcal{M}: \frac{\cos \alpha}{2|\mathcal{K}|} \leq f^{+}(v) \leq \frac{\cos \alpha}{|\mathcal{K}|}\right\}
$$

Os cones acima são usados para definir por partes um campo de cone invariante $C:=\{C(x)\}_{x}$. Para cada $x=(s, \alpha)$, a escolha $C(x):=C_{i}(x)$ irá depender sobre $s, s_{-1}$, e o que ocorre com a trajetória entre as colisões em $s_{-1}$ e $s$.

(A) Se $s \in \Gamma^{-}$, defina $C(x):=C_{0}(x)$.

(B) Se $s \in \Gamma^{+}$, então existem dois subcasos:

(B.1) Se $s_{-1} \in \Gamma^{+}$, defina $C(x):=C_{2}(x)$.

(B.2) Se $s_{-1} \in \Gamma^{-}$, há ainda dois subcasos, dependendo se o pedaço da trajetória entre $s_{-1}$ e $s$ possui colisões com $\Gamma^{0}$ :

(B.2.1) Nenhuma colisão com $\Gamma^{0}$ entre $s_{-1}$ e $s$ : defina $C(x):=C_{1}(x)$.

(B.2.2) Pelo menos uma colisão com $\Gamma^{0}$ entre $s_{-1}$ e $s$ : defina $C(x):=C_{2}(x)$.

Observamos que $C(x)$ é uma função mensurável de $x$.

Teorema 3. (Bussolari-Lenci, [BL08]) O campo de cone $C$ definido acima é certamente estritamente invariante relativo à aplicação $\mathcal{F}$.

Dessa forma concluímos que $\mathcal{F}$ é hiperbólica.

A principal questão que levantamos nesse trabalho é: a aplicação $\mathcal{F}$ é ergódica? A resposta é sim sob certas condições. 


\section{Capítulo 4}

\section{Ergodicidade Local.}

Quase todas as provas de ergodicidade para sistemas hiperbólicos estão baseadas no método de Hopf [Hop39] que serão introduzidas aqui informalmente, deixando as formalidades para a referência [CM06].

\subsection{Método de Hopf}

Suponhamos que $F: M \rightarrow M$ é uma transformação suave, hiperbólica que preserva a medida de probabilidade $\mu$. Suponhamos ainda que para cada $x \in M$, exista uma variedade estável $W^{s}(x)$ e uma variedade instável $W^{u}(x)$. A prova da afirmação que fazemos abaixo pode ser encontrada nos detalhes em [CM06].

Afirmação 1. Quase toda variedade estável e instável pertence (mod 0) a uma componente ergódica da transformação $F$.

Considere $W_{1}, W_{2}, \ldots, W_{n}$ uma sequência de variedades estáveis e instáveis tais que $W_{i} \cap W_{i+1} \neq$ $\emptyset$ para todo $i=1, \ldots, n-1$. Isso é possível se as variedades estáveis e instáveis se alternam. Se essas variedades são típicas e se elas intersectam em pontos típicos, então pela afirmação acima, a união $\cup W_{i}$ pertence $(\bmod 0)$ a uma componente ergódica de $F$.

A sequência de variedades estáveis e instáveis é chamada de cadeia de Hopf ou um zig zag. Tal sequência é um instrumento na construção de componentes ergódicas para transformações hiperbólicas.

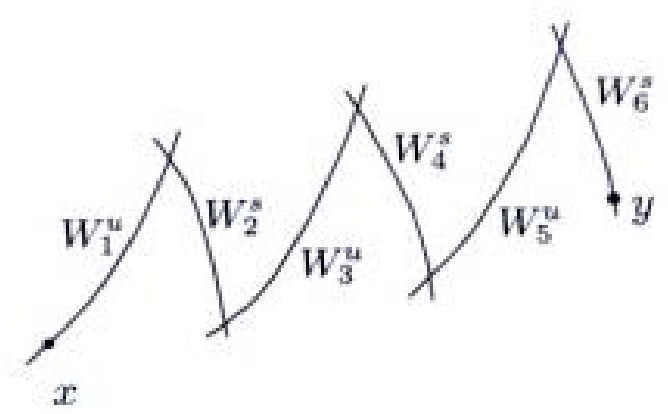

Figura 4.1: Uma cadeia de Hopf conectando $x$ e $y$.

Seja $A \subset M$ tal que quaisquer dois pontos típicos $x, y \in A$ podem ser conectados por uma cadeia de Hopf como na figura anterior. Então $A$ pertence $(\bmod 0)$ a uma componente ergódica de $F$. Se $A=M$, então a transformação $F$ é ergódica.

Dessa forma, o método de Hopf se concentra no seguinte fato: para provar a ergodicidade de uma transformação hiperbólica $F$, é suficiente mostrar que quaisquer dois pontos típicos $x, y \in M$ podem ser conectados por uma cadeia de Hopf de variedades estáveis e instáveis típicas intersectando-se em 
pontos típicos. Para transformações não ergódicas, o método de Hopf pode ser usado para identificar suas componentes ergódicas.

O setting que permite a construção de uma cadeia de Hopf, chamadas de condições de KatokStrelcyn, são listadas em [CM06] como (H1)-(H6) na Seção 6.3. A saber, todas essas hipóteses são satisfeitas em nosso bilhar.

Para provarmos a ergodicidade da transformação $F$, pode-se empregar a seguinte estratégia:

- Encontrar um conjunto $M_{1} \subset M$ tal que para cada ponto $x \in M_{1}$, existe uma vizinhança $U(x)$ que pertence $(\bmod 0)$ a uma componente ergódica de $F$.

- Mostrar que $M_{1}$ tem medida total e é conexo por caminhos.

O primeiro passo é conhecido como ergodicidade 'local', enquanto que o segundo como ergodicidade 'global'.

\subsection{Principais resultados}

Primeiramente, notamos que as técnicas utilizadas nas provas da ergodicidade, estão baseadas nos trabalhos de Del Magno-Markarian [MM14]. Dessa forma, necessitamos trabalhar em todo o bilhar.

Denotamos por $M=\mathcal{M}^{+} \cup \mathcal{M}^{-} \cup \mathcal{M}^{0}$ e $\mathcal{M}=\mathcal{M}^{+} \cup \mathcal{M}^{-}$e consideramos $\mathfrak{F}: M \longrightarrow M$ a aplicação do bilhar definido em todo espaço de fase $M$ com a propriedade que $\mathcal{F} x=\mathfrak{F}^{k(x)} x$, $x \in \mathcal{M}$, onde $\mathfrak{F}^{n} x \in \mathcal{M}^{0}$, para $0<n<k(x)$, com $k(x) \neq 1$. Aqui $\mathcal{F}$ denota a aplicação do bilhar de Bussolari-Lenci.

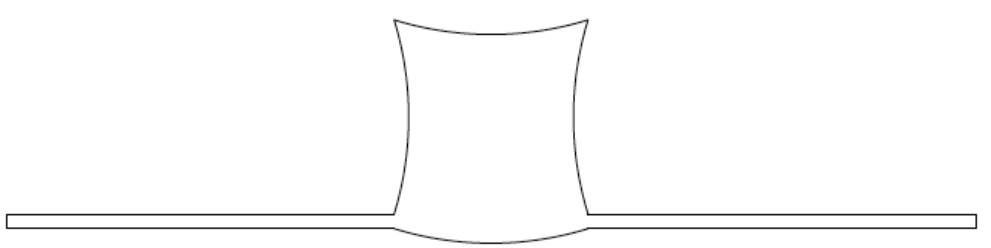

Figura 4.2: A principal mesa de bilhar.

A Figura 4.2 mostra a mesa de bilhar que estamos interessados para o resto do trabalho. Por [BL08], sabemos que $\mathcal{F}$ é hiperbólica, o que implica pelo teorema espectral (veja Teorema 5 no Apêndice A) que $\mathcal{F}$ tem no máximo um número enumerável de componentes ergódicas de medida positiva (para a medida natural $\mu$ ) com cada componente ergódica decomposta em um número finito de componentes Bernoulli ciclicamente permutadas por $\mathcal{F}$. Nós provamos que, existe um conjunto $\mathcal{H} \subset \mathcal{M}$ de medida total tal que, para todo $x \in \mathcal{H}$, existe uma vizinhança $U \subset \mathcal{M}$ contida $(\bmod 0)$ em uma componente Bernoulli de $\mathcal{F}$ (veja Teorema A). Por essa razão, esse tipo de resultado é as vezes chamado de Teorema Ergódico Local. Por um argumento de conexidade, finalmente, mostramos que $\mathcal{F}$ é Bernoulli.

Definimos $\mathcal{H}:=\mathcal{M} \backslash \mathcal{R}$. Vemos que $\mu(\mathcal{H})=1$, pois pela regularidade do conjunto $\mathcal{R}_{k}^{+}$(veja Subseção 4.3.1), concluimos que $\mu\left(\mathcal{R}_{k}^{+}\right)=0$.

O principal resultado desse trabalho é o seguinte teorema e será provado na próxima seção:

Teorema A. Cada ponto $x \in \mathcal{H}$ possui uma vizinhança contida (mod 0$)$ em uma componente Bernoulli de $\mathcal{F}$.

Corolário 1. Cada componente Bernoulli de $\mathcal{F}$ é aberta $(\bmod 0)$.

Demonstração. Seja $B$ uma componente Bernoulli. Como $\mu(B)>0$, então $\mu(B \cap \mathcal{H})>0$. Seja $x \in B \cap \mathcal{H}$, e seja $U$ a vizinhança de $x$ como no Teorema A. O conjunto $V:=\bigcup_{n \in \mathbb{Z}} \mathcal{F}^{n} U$ é aberto. Além disso, como $V$ é invariante e contido $(\bmod 0)$ em $B$, segue que $B=V(\bmod 0)$. 
Corolário 2. A aplicação $\mathcal{F}: \mathcal{H} \longrightarrow \mathcal{H}$ é Bernoulli.

Antes de mostrarmos o corolário, precisamos da proposição a seguir. Lembre que $\mathcal{H}$ consiste de componentes conexas $\mathcal{M}_{i}$.

Proposição 2. Cada componente conexa $\mathcal{M}_{i}$ do espaço $\mathcal{H}$ pertence $(\bmod 0)$ a uma componente Bernoulli de $\mathcal{F}$.

Demonstração. Como $\mathcal{R}$ é enumerável (veja [MM13b]), então o complementar $\mathcal{M}_{i} \backslash \mathcal{R}$ é conexo por caminhos. Suponhamos que $x, y \in \mathcal{M}_{i} \backslash \mathcal{R}$ são conectados por uma curva contínua compacta $\mathcal{C} \subset \mathcal{M}_{i} \backslash \mathcal{R}$. Cada ponto $z \in \mathcal{C}$ satisfaz o Teorema A, assim existe vizinhança $U_{z}$ pertencente $(\bmod 0)$ a uma componente Bernoulli de $\mathcal{F}$. Devido a compacidade de $\mathcal{C}$, pode-se cobrir por um número finito de tais vizinhanças abertas, veja figura abaixo.

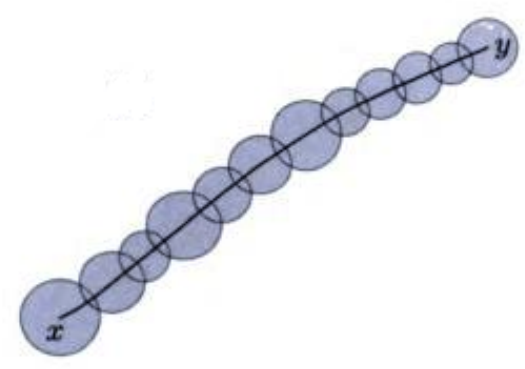

Figura 4.3: Prova da Proposição 2.

Nessas condições, demonstramos o Corolário 2.

Demonstração. Pelo Teorema A, cada ponto de $\mathcal{H}$ possui uma vizinhança contida, a menos de um conjunto de medida nula, em uma componente Bernoulli de $\mathcal{F}$. Pela Proposição 2, o mesmo é verdade para cada componente conexa de $\mathcal{H}$; e então para cada $\mathcal{M}_{i} \cap \mathcal{H}$ tal que $\mathcal{M}_{i} \subset \mathcal{M}^{-} \cup \mathcal{M}^{+}$. Como $\mu(\mathcal{H})=1$, podemos concluir que cada $\mathcal{M}_{i} \subset \mathcal{M}^{-} \cup \mathcal{M}^{+}$está contido $(\bmod 0)$ em uma única componente Bernoulli de $\mathcal{F}$. Como $\Omega$ é conexo, segue que todos $\mathcal{M}_{i}$ pertencem a mesma componente Bernoulli, ou seja, $\mathcal{F}$ é Bernoulli.

\subsection{Ergodicidade local}

Nessa seção, enunciaremos os principais resultados para a prova do Teorema A. Isso será feito aplicando o Teorema 4 à aplicação $\mathcal{F}$. Inicialmente, enunciaremos o Teorema 4 e em seguida daremos as noções requeridas para a sua formulação. Esse teorema é a versão para bilhares planares de um Teorema Ergódico Local para simplectomorfismos com singularidades em qualquer dimensão provado em [MM13a]. O Teorema 4 possui quatro hipóteses chamadas de Condições L1-L4.

Demonstração do Teorema A. A conclusão do Teorema segue aplicando o Teorema 4 aos pontos de $\mathcal{H}$. Para isso, precisamos mostrar que cada ponto de $\mathcal{H}$ é suficiente (veja Definição 7), e que para cada um desses pontos, as Condições L1-L4 do Teorema 4 são satisfeitas. O primeiro fato é provado no Corolário 3, enquanto que o segundo fato está provado nas Proposições 3, 4, 6 e 13.

Teorema 4. (Teorema Ergódico Local.) Suponha que L1 é satisfeita, e que $x \in \mathcal{M} \backslash \partial \mathcal{M}$ é um ponto suficiente satisfazendo L2-L4. Então existe uma vizinhança de $x$ contida, a menos de um conjunto de $\mu$-medida nula, em uma componente Bernoulli de $\mathcal{F}$. 


\subsubsection{Condição L1 - Regularidade}

Definição 4. Um conjunto $X \subset \mathcal{M}$ é dito regular se $X$ é uma união finita de arcos de classe $C^{2}$ que intersectam somente nas extremidades. A coleção de tais arcos é chamada uma decomposição de $X$.

Definição 5. Dizemos que a Condição L1 é satisfeita se os conjuntos $\mathcal{R}_{k}^{+}$e $\mathcal{R}_{k}^{-}$são regulares para cada $k>0$.

Antes de garantirmos a regularidade dos conjuntos $\mathcal{R}_{k}^{+}$e $\mathcal{R}_{k}^{-}$, definimos o conceito de arco singular e daremos um novo sistema de coordenadas locais para $\mathcal{M}$ que foram introduzidos em [MM13b].

\section{Arcos singulares}

A grosso modo, um arco singular é um arco de classe $C^{2}$ contido em $\mathcal{R}_{m}^{+}$para algum $m>0$.

Definição 6. Um arco $\Sigma$ de classe $C^{2}$ é chamado negativamente singular se ou $\Sigma \subset \partial \mathcal{M}$ ou se existem $j>0$ e arcos $\Sigma_{0}, \ldots, \Sigma_{j-1}$ tais que após definir $\Sigma_{j}=\Sigma$ tem-se:

- $\Sigma_{0} \subset \partial \mathcal{M}$;

- $\Sigma_{i} \cap \mathcal{S}_{1}^{+} \subset \partial \Sigma_{i}$ para $0 \leq i<j$;

- int $_{i+1}=\mathcal{F}\left(\right.$ int $\left.\Sigma_{i}\right)$ para $0 \leq i<j$;

- $\Sigma_{i} \cap \partial \mathcal{M} \subset \partial \Sigma_{i}$ para $0 \leq i \leq j$.

Um arco positivamente singular é definido similarmente substituindo $\mathcal{F}$ com $\mathcal{F}^{-1}$.

\section{Coordenadas locais para $\mathcal{M}$}

Suponhamos que $\Sigma_{1}$ é um arco negativamente singular tal que $\Sigma_{1} \cap \mathcal{S}_{1}^{+} \subset \partial \Sigma_{1}$. Nesse caso, cada conjunto $q\left(\Sigma_{1}\right)$ e $q_{1}\left(\right.$ int $\left.\Sigma_{1}\right)$ está contido em uma única componente de $\partial \Omega$. Sejam $\Gamma_{1}$ e $\Gamma_{2}$ as componentes de fronteira contendo $q\left(\Sigma_{1}\right)$ e $q_{1}\left(\right.$ int $\left.\Sigma_{1}\right)$, respectivamente.

Dado $z_{1} \in \Sigma_{1}$, sejam $p_{1}=q\left(z_{1}\right)$ e $p_{2}$ o ponto de interseção entre $\Gamma_{2}$ e o raio emergindo de $z_{1}$ tendo a distância mínima de $p_{1}$. Estudamos agora, a imagem de $\Sigma_{1}$ sob a aplicação do bilhar $\mathcal{F}$ para todas as possíveis escolhas de $\Gamma_{1}$ e $\Gamma_{2}$. Para efeito de cálculo, é conveniente obter uma descrição analítica da configuração formada por $\Gamma_{1}$ e $\Gamma_{2}$ em um sistema de coordenadas cartesianas $(X, Y)$ para $\mathbb{R}^{2}$. Ao fazer isso, obtemos um novo sistema de coordenadas locais para $\mathcal{M}$. Podemos escolher o sistema de coordenadas cartesianas $(X, Y)$ (veja Fig.4.4) tal que

- $p_{1}=(0,0)$ e $p_{2}=(\bar{X}, 0)$ com $\bar{X} \leq 0$ em coordenadas $(X, Y)$;

- existem dois intervalos fechados $U_{1}$ e $U_{2}$ de $\mathbb{R}$ com $U_{1}$ contendo 0 e $U_{2}$ contendo $\bar{X}$ e duas funções $C^{3} f_{1}: U_{1} \rightarrow \mathbb{R}$ e $f_{2}: U_{2} \rightarrow \mathbb{R}$ com $f_{1}(0)=f_{2}(\bar{X})=0$ tal que $\Gamma_{1}=\operatorname{graf}\left(f_{1}\right)$ e $\Gamma_{2}=\operatorname{graf}\left(f_{2}\right)$; além disso, temos ou $f_{1}^{\prime \prime} \equiv 0$ ( $\Gamma_{1}$ flat) ou $f_{1}^{\prime \prime}>0$ em $U_{1}$ ( $\Gamma_{1}$ curva), e/ou $f_{2}^{\prime \prime} \equiv 0$ ( $\Gamma_{2}$ flat $)$ ou $\left|f_{2}^{\prime \prime}\right|>0$ em $U_{2}\left(\Gamma_{2}\right.$ curva $)$.

Dado $x \in \mathcal{M}_{1} \cup \mathcal{M}_{2}$, seja $X$ a $X$-coordenada de $q(x)$, e seja $\delta \in[0,2 \pi)$ o ângulo entre o eixo $X$ e o vetor $u(x)$. Recorde que $\mathcal{M}_{1} \cup \mathcal{M}_{2}$ está equipado com as coordenadas locais $(s, \alpha)$. O par $(X, \delta)$ forma outro sistema de coordenadas locais em $\mathcal{M}_{1} \cup \mathcal{M}_{2}$. Em coordenadas $(X, \delta)$ temos $z_{1}=(0, \pi)$. A relação entre $(s, \alpha)$ e $(X, \delta)$ em $\mathcal{M}_{1}$ segue como na equação (17) de [MM13b], difere apenas com relação a parametrização do ângulo:

$$
\begin{aligned}
s & =\int_{0}^{X} \sqrt{1+f_{1}^{\prime 2}(\tilde{X})} d \tilde{X} \\
\alpha & =\left\{\begin{array}{ccc}
\delta-\tan ^{-1} f_{1}^{\prime}(X)-\pi / 2, & \text { se } & f_{1}^{\prime}(X) \geq 0, \\
-\delta+\tan ^{-1} f_{1}^{\prime}(X)+3 \pi / 2, & \text { se } & f_{1}^{\prime}(X)<0 .
\end{array}\right.
\end{aligned}
$$




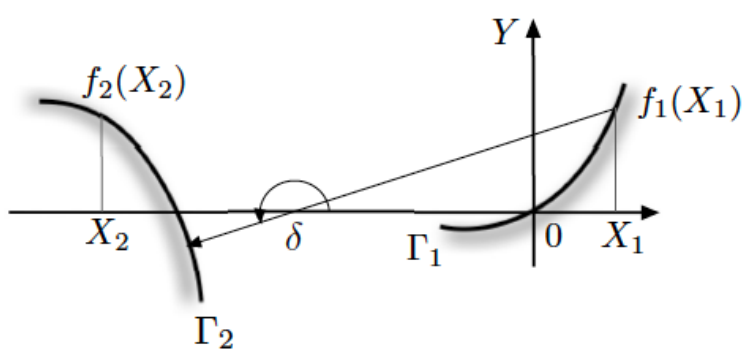

Figura 4.4: $\Gamma_{1}$ e $\Gamma_{2}$ no sistema de coordenadas cartesianas $(X, Y)$.

A transformação é uma $C^{2}$ mudança de coordenadas.

Como o arco $\Sigma_{1}$ é um intervalo fechado mergulhado, existe um intervalo fechado $I_{1}=\left[a_{1}, b_{1}\right]$ com $a_{1}<b_{1}$ e duas funções $C^{2} X_{1}: I_{1} \rightarrow \mathbb{R}$ e $\delta_{1}: I_{1} \rightarrow \mathbb{R} \operatorname{com}\left(X_{1}^{\prime}(a), \delta_{1}^{\prime}(a)\right) \neq(0,0)$ para todo $a \in I_{1}$ tal que $\gamma_{1}:=\left(X_{1}, \delta_{1}\right): I_{1} \rightarrow \Sigma_{1}$ é um $C^{2}$ difeomorfismo. Escolha $\bar{a} \in\left[a_{1}, b_{1}\right]$ tal que $\gamma_{1}(\bar{a})=z_{1}$. Pela escolha do sistema de coordenadas $(X, Y)$, segue que $X_{1}(\bar{a})=0$ e $\delta_{1}(\bar{a})=\pi$.

No próximo lema, é dado a expressão do tempo de focalização de $\gamma_{1}^{\prime}(a)$ em coordenadas $(X, \delta)$, veja Lema 6.3 de [MM13b].

Lema 4. Temos

$$
f^{+}\left(\gamma_{1}(a), \gamma_{1}^{\prime}(a)\right)=\left\{\begin{array}{cll}
{\left[\tan \delta_{1}(a)-f_{1}^{\prime}\left(X_{1}(a)\right)\right] \cos \delta_{1}(a) \frac{X_{1}^{\prime}(a)}{\delta_{1}^{\prime}(a)},} & \text { se } & \delta_{1}^{\prime}(a) \neq 0 \\
\infty, \text { se } & \delta_{1}^{\prime}(a)=0
\end{array}\right.
$$

A relação entre duas colisões consecutivas, a primeira ocorrendo em $\Gamma_{1}$ e a segunda em $\Gamma_{2}$, pode ser expressa como zeros simultâneos das funções $F: \mathcal{M}_{1} \times \mathcal{M}_{2} \rightarrow \mathbb{R}$ e $G: \mathcal{M}_{1} \times \mathcal{M}_{2} \rightarrow \mathbb{R}$ definidas por

$$
\begin{aligned}
& F\left(X_{1}, \delta_{1}, X_{2}, \delta_{2}\right)=f_{1}\left(X_{1}\right)-f_{2}\left(X_{2}\right)-\tan \delta_{1} \cdot\left(X_{1}-X_{2}\right) \\
& G\left(X_{1}, \delta_{1}, X_{2}, \delta_{2}\right)=\delta_{1}+\delta_{2}-2 \alpha_{2}\left(X_{2}\right)+\pi
\end{aligned}
$$

para cada $\left(X_{1}, \delta_{1}, X_{2}, \delta_{2}\right) \in \mathcal{M}_{1} \times \mathcal{M}_{2}$. A função $\alpha_{2}$ denota o ângulo formado pelo eixo $X$ com a normal à $\Gamma_{2} \mathrm{em}\left(X_{2}, f_{2}\left(X_{2}\right)\right)$ apontando para dentro do domínio do bilhar $\Omega$. A relação $F\left(X_{1}, X_{2}, \delta_{1}, \delta_{2}\right)=$ 0 é a equação da reta tendo inclinação igual à $\tan \delta_{1}$ e passando pelos pontos $\left(X_{1}, f_{1}\left(X_{1}\right)\right)$ e $\left(X_{2}, f_{2}\left(X_{2}\right)\right)$, enquanto que a relação $G\left(X_{1}, X_{2}, \delta_{1}, \delta_{2}\right)=0$ descreve a igualdade dos ângulos de incidência e reflexão em $\left(X_{2}, f_{2}\left(X_{2}\right)\right)$.

Recorde que $I_{1} \ni a \mapsto\left(X_{1}(a), \delta_{1}(a)\right)$ é uma parametrização do arco singular $\Sigma_{1}$. Sejam $F_{1}$ : $\mathcal{M}_{2} \times I_{1} \rightarrow \mathbb{R}$ e $G_{1}: \mathcal{M}_{2} \times I_{1} \rightarrow \mathbb{R}$ funções $C^{2}$ diferenciáveis dadas por:

$$
\begin{aligned}
F_{1}\left(X_{1}, \delta_{1}, X_{2}, \delta_{2}\right) & =F_{1}\left(X_{1}(a), \delta_{1}(a), X_{2}, \delta_{2}\right), \\
G_{1}\left(X_{1}, \delta_{1}, X_{2}, \delta_{2}\right) & =G_{1}\left(X_{1}(a), \delta_{1}(a), X_{2}, \delta_{2}\right),
\end{aligned}
$$

para cada $\left(X_{2}, \delta_{2}, a\right) \in \mathcal{M}_{2} \times I_{1}$. Defina também:

$$
\begin{aligned}
D_{1}\left(X_{2}, \delta_{2}, a\right) & =\operatorname{det}\left(\begin{array}{cc}
\partial_{\delta_{2}} F_{1} & \partial_{a} F_{1} \\
\partial_{\delta_{2}} G_{1} & \partial_{a} G_{1}
\end{array}\right) \\
& =\left[\tan \delta_{1}(a)-f_{1}^{\prime}\left(X_{1}(a)\right)\right] X_{1}^{\prime}(a)+\frac{\delta_{1}^{\prime}(a)}{\cos ^{2} \delta_{1}(a)},
\end{aligned}
$$

para cada $\left(X_{2}, \delta_{2}, a\right) \in \mathcal{M}_{2} \times I_{1}$. Vemos que $D_{1}$ está bem definida, pois de acordo com nosso setting, sempre temos $\cos \delta_{1} \neq 0$ para $a$ suficientemente próximo de $\bar{a}$.

O próximo lema, nos dá a relação entre $D_{1}\left(X_{2}, \delta_{2}, a\right)$ e algumas quantidades geométricas asso- 
ciadas à colisão $\gamma_{1}(a)$. Para a sua prova, veja Lema 6.6 de [MM13b]. Recorde que $\tau\left(\gamma_{1}(a)\right)$ denota o comprimento do segmento conectando $q\left(\gamma_{1}(a)\right)$ e $q_{1}\left(\gamma_{1}(a)\right)$.

Lema 5. Temos

$$
D_{1}\left(X_{2}, \delta_{2}, a\right)=\left\{\begin{array}{crr}
{\left[f^{+}\left(\gamma_{1}(a), \gamma_{1}^{\prime}(a)\right)-\tau\left(\gamma_{1}(a)\right)\right] \frac{\delta_{1}^{\prime}(a)}{\cos \delta_{1}(a)},} & \text { se } & \delta_{1}^{\prime}(a) \neq 0 \\
{\left[\tan \delta_{1}(a)-f_{1}^{\prime}\left(X_{1}(a)\right)\right] X_{1}^{\prime}(a), \text { se }} & & \delta_{1}^{\prime}(a)=0
\end{array}\right.
$$

Proposição 3. A Condição L1 é satisfeita.

Demonstração. Dado um arco $\Sigma_{1} \subset \mathcal{R}_{k}^{ \pm}$para algum $k>0$, a proposição será provada no sistema de coordenadas cartesianas $(X, Y)$ estudado acima. Para garantir que o arco $\Sigma_{1}$ é de classe $C^{2}$, usaremos o Teorema da Função Implícita. Assim, devemos garantir que o determinante da derivada (Lema 5) é diferente de zero nos pontos da imagem de $\Sigma_{1}$. Primeiramente vamos analisar o caso em que $\delta_{1}^{\prime}(a) \neq 0$, isso se reduz a estudarmos a diferença $f_{j}^{+}-\tau_{j-1}$ para algum $1 \leq j \leq k$. É suficiente provar a proposição somente para os conjuntos $\mathcal{R}_{k}^{+}$. De fato, o resultado se estende para $\mathcal{R}_{k}^{-}$pelo tempo de reversibilidade de $\mathcal{F}$.

Assim, dado $\Sigma_{1} \subset \mathcal{R}_{k}^{+}$para algum $k>0$, considere $z \in-E_{i}$ para algum $i$. Seja $U_{z}$ vizinhança de $z$ e tome $x=(s, \alpha) \in-E_{i} \cap U_{z} \cap \Sigma_{1} \backslash A_{2}$, assim existe $1 \leq j \leq k$ tal que $\mathcal{F}^{j} x=x_{j}=\left(s_{j}, \alpha_{j}\right) \in E_{j}$.

Estudemos os casos possíveis:

1. $x_{j-1} \in \mathcal{M}^{-}$e $x_{j} \in \mathcal{M}^{-}$: Nesse caso, $C\left(x_{j-1}\right)=C_{0}\left(x_{j-1}\right)$ e $C\left(x_{j}\right)=C_{0}\left(x_{j}\right)$. Ou seja, $F_{j}^{+} \in D_{-2}^{\circ}\left(s_{j}\right)$ e, portanto, $f_{j}^{+}<0$. Como $\tau_{j-1} \gg 0$, temos $\tau_{j-1} \gg f_{j}^{+}$.

2. $x_{j-1} \in \mathcal{M}^{-}$e $x_{j} \in \mathcal{M}^{+}$: Aqui $C\left(x_{j}\right)=C_{0}\left(x_{j}\right)$, porém o cone $C\left(x_{j}\right)$ pode ter duas formas. Se não houver colisão com $\Gamma^{0}$ entre $s_{j-1}$ e $s_{j}$, então $C\left(x_{j}\right)=C_{1}\left(x_{j}\right)$ daí $F_{j}^{+} \in D_{0^{-}}\left(s_{j}\right)$ i.é $f_{j}^{+} \leq 0$. Novamente, como $\tau_{j-1} \gg 0$ segue o resultado. Se há pelo menos uma colisão com $\Gamma^{0}$ entre $s_{j-1}$ e $s_{j}$ então $C\left(x_{j}\right)=C_{2}\left(x_{j}\right)$, assim $F_{j}^{+} \in D_{2}^{\circ}\left(s_{j}\right) \backslash D_{4}\left(s_{j}\right)$, isto é, $f_{j}^{+}<\cos \alpha / \mathcal{K}_{f} \leq 1 / \mathcal{K}_{f}$, porém $\tau>2 l \geq 2 / \mathcal{K}_{f}$, que implica $\tau_{j-1} \gg f_{j}^{+}$.

3. $x \in \mathcal{M}^{+}$e $\mathcal{F}^{j} x \in \mathcal{M}^{-}$: Por definição, $C\left(x_{j}\right)=C_{0}\left(x_{j}\right)$ (se houver ou não colisão com $\Gamma^{0}$ ). Assim, $f_{j}^{+} \leq 0 \operatorname{logo} \tau \gg f_{j}^{+}$.

4. $x_{j-1} \in \mathcal{M}^{+}$e $x_{j} \in \mathcal{M}^{+}$: Aqui $C\left(x_{j}\right)=C_{2}\left(x_{j}\right)$ por definição, assim $f_{j}^{+} \leq \cos \alpha / \mathcal{K}_{f}$. E temos dois subcasos: se não houver colisão com $\Gamma^{0}$ entre $s_{j-1}$ e $s_{j}$ então o pedaço da trajetória é uma corda de comprimento $\tau_{j-1}=2(\cos \alpha) / \mathcal{K}_{f}>\cos \alpha / \mathcal{K}_{f}$. Notamos que nesse caso $\cos \alpha \gg 0$, uma vez que as colisões sucessivas contra a componente focalizadora produzem ângulos iguais aos de entrada. Se a entrada na focalizadora é proveniente da componente flat, o ângulo $\alpha_{j}$ poderá estar próximo de $\pi / 2$, porém a trajetória deverá ir novamente para a parte flat, mas isso somente ocorre em um número finito de vezes, dessa forma, basta tomar o ângulo $\alpha_{j}$ em que a trajetória vai para a parte dispersora. Se a entrada na focalizadora é proveniente da dispersora é trivial. Portanto, $\tau_{j-1} \gg f_{j}^{+}$. Se houver pelo menos uma colisão com $\Gamma^{0}$ entre $s_{j-1}$ e $s_{j}$ então $\tau_{j-1}>2 l \geq 2 / \mathcal{K}_{f}$, ou seja, $\tau_{j-1} \gg \cos \alpha / \mathcal{K}_{f}$ que implica $\tau_{j-1} \gg f_{j}^{+}$.

Suponhamos agora que $\delta_{1}^{\prime}(a)=0$. Se $X_{1}^{\prime}(a)=0$ então $\gamma_{1}^{\prime}(a)=\left(X_{1}^{\prime}(a), \delta_{1}^{\prime}(a)\right)=(0,0)$ o que não ocorre pela parametrização do arco singular $\Sigma_{1}$. Agora, por construção do sistema de coordenadas segue que $f_{1}^{\prime}\left(X_{1}(a)\right) \neq \tan \delta_{1}(a)$. Portanto, o determinante da derivada dado no Lema 5 é diferente de zero.

Observação 4. Notamos que a Proposição 3 implica que existem constantes positivas $C$ e a tais que $\mu\left(\mathcal{R}_{1}^{ \pm}(\epsilon)\right) \leq C \epsilon^{a}$, para cada $\epsilon>0$ suficientemente pequeno. 


\subsubsection{Condição L2 - Alinhamento}

Definição 7. Um ponto $x \in \mathcal{M} \backslash \partial \mathcal{M}$ é chamado suficiente se existem $l \in \mathbb{Z}, N \in \mathbb{N}, O$ aberto $e$ um campo de cone invariante $\left(O \cup \mathcal{F}^{-N} O, K\right)$ tais que:

1. $x \notin \mathcal{R}_{|l|}^{+} \cap \mathcal{R}_{|l|}^{-}$,

2. $O$ é uma vizinhança de $\mathcal{F}^{l+N} x$ e $O \cap \mathcal{R}_{N}^{-}=\emptyset$,

3. $\sigma_{K}\left(D_{y} \mathcal{F}^{N}\right)>3$ para todo $y \in \mathcal{F}^{-N} O$.

Dizemos que $x$ é um ponto suficiente com quádrupla $(l, N, O, K)$.

No Corolário 3 iremos provar que todo $x \in \mathcal{H}$ é suficiente, assim supomos aqui a existência de uma quádrupla $(l, N, O, K)$ associada.

Definição 8. Dizemos que $x$ satisfaz a Condição L2 se o subespaço tangente $T_{y} \Sigma$ está contido em $K(y)\left(\right.$ resp. $\left.K^{\prime}(y)\right)$ para cada $k>0$, cada $C^{2}$-arco $\Sigma$ de $R_{k}^{-}$(resp. $R_{k}^{+}$) e cada $y \in \Sigma \cap \mathcal{F}^{-N} O$ (resp. $\Sigma \cap O)$.

Proposição 4. Cada ponto $x \in \mathcal{H}$ satisfaz a Condição L2.

Demonstração. Seja $\Sigma \subset \mathcal{R}_{k}^{-}$um arco $C^{2}$ com $k>0$. Podemos supor sem perda de generalidade que $\Sigma \not \subset \partial \mathcal{M}$, pois caso contrário teríamos $\Sigma \cap \mathcal{F}^{-N} O=\emptyset$ pela condição (2) da Definição 7 .

A idéia da prova é estudar a evolução de um feixe de trajetórias emergindo de um vértice (ou tangência). Denotemos tal vértice por $z$. Ou seja, basta analisarmos a imagem do vetor $v=$ $(d s, d \alpha)=(0,1) \in T_{z} \mathcal{M}$ (ou $v=(1,0) \in T_{z} \mathcal{M}$ de modo análogo) em diferentes componentes. Assim, seja $y \in \Sigma \cap \mathcal{F}^{-N} O$ tal que $y=\mathcal{F} z=\left(d s_{1}, d \alpha_{1}\right)$. Utilizando a fórmula (2.5) da diferencial de $\mathcal{F}$, obtemos

$$
D_{z} \mathcal{F} v=\left(d s_{1}, d \alpha_{1}\right)=\left(\frac{-\tau}{\cos \alpha_{1}}, \frac{\tau \mathcal{K}}{\cos \alpha_{1}}-1\right) \Rightarrow \frac{d \alpha_{1}}{d s_{1}}=-\mathcal{K}+\frac{\cos \alpha_{1}}{\tau} .
$$

Logo, pela fórmula (2.2), tem-se

$$
f_{1}^{+}(v)=\frac{\tau \cos \alpha_{1}}{2 \mathcal{K} \tau-\cos \alpha_{1}} .
$$

(I) Se $z=(0, \alpha) \in \mathcal{M}_{1}^{-} \cap \mathcal{M}_{2}^{-}$e $y \in \mathcal{M}_{3}^{-}$, temos por definição que $K(y):=C_{0}(y)$. Logo devemos provar que $D_{z} \mathcal{F} v \in C_{0}(y)$. De fato, como $\mathcal{K}_{d}<0$ temos $2 \mathcal{K}_{d} \tau-\cos \alpha_{1}<0$ e assim $f_{1}^{+}(v) \leq 0$. Agora, suponhamos que

$$
f_{1}^{+}(v)=\frac{\tau \cos \alpha_{1}}{2 \mathcal{K}_{d} \tau-\cos \alpha_{1}}<\frac{\cos \alpha_{1}}{\mathcal{K}_{d}}
$$

isso implicaria $\left(\cos \alpha_{1}\right) / \mathcal{K}_{d}>\tau>0$ com $\mathcal{K}_{d}<0$, o que é um absurdo. Logo, $f_{1}^{+}(v) \geq\left(\cos \alpha_{1}\right) / \mathcal{K}_{d}$. (II) Se $z=(0, \alpha) \in \mathcal{M}_{1}^{-} \cap \mathcal{M}_{2}^{-}$e $y \in \mathcal{M}^{+}$, então $K(y)$ pode ter duas formas:

(II.I) se não houver colisão com $\Gamma^{0}$ entre $s=0$ e $s_{1}$ então $K(y):=C_{1}(y)$. Nesse caso, $\mathcal{K}_{f}>0$ porém pela propriedade (3.1) segue que $z \in D_{4}(y)$, isso significa que o comprimento do segmento determinado por $y$ e $\partial D_{4}(y)$ ao qual $z$ pertence, é maior que $\tau$. Em outras palavras, $\left(2 \cos \alpha_{1}\right) / 4 \mathcal{K}_{f}>$ $\tau$, ou seja, $2 \mathcal{K}_{f} \tau-\cos \alpha_{1}<0$, e portanto, $f_{1}^{+}(v) \leq 0$.

(II.II) se houver pelo menos uma colisão com $\Gamma^{0}$ entre $s=0$ e $s_{1}$, então $K(y):=C_{2}(y)$. Aqui $\tau>2 l \geq 2 / \mathcal{K}_{f}$, pois $l \geq 1 / \mathcal{K}_{f}$. Assim,

$$
2 \tau \mathcal{K}_{f}-\cos \alpha_{1}>4-\cos \alpha_{1}>\tau \mathcal{K}_{f} \Rightarrow \frac{1}{2 \tau \mathcal{K}_{f}-\cos \alpha_{1}}<\frac{1}{\tau \mathcal{K}_{f}}
$$

daí,

$$
f_{1}^{+}(v)=\frac{\tau \cos \alpha_{1}}{2 \tau \mathcal{K}_{f}-\cos \alpha_{1}} \leq \frac{\cos \alpha_{1}}{\mathcal{K}_{f}}
$$


Agora,

$$
\frac{2}{\mathcal{K}_{f}}<\tau \Rightarrow \frac{1}{4} \frac{2}{\mathcal{K}_{f}}<\frac{1}{4} \tau \Rightarrow \frac{\cos \alpha_{1}}{2 \mathcal{K}_{f}}<\frac{\tau \cos \alpha_{1}}{4} \leq \frac{\tau \cos \alpha_{1}}{2 \tau \mathcal{K}_{f}-\cos \alpha_{1}}=f_{1}^{+}(v),
$$

uma vez que $2 \tau \mathcal{K}_{1}-\cos \alpha_{1} \leq 4$.

(III) Se $z=(0, \alpha) \in \mathcal{M}^{0} \cap \mathcal{M}^{+}$e $y \in \mathcal{M}^{-}$, então $K(y):=C_{0}(y)$ e segue de modo análogo ao caso (I) (com ou sem colisão com $\Gamma^{0}$ ).

(IV) Se $z=(0, \alpha) \in \mathcal{M}^{0} \cap \mathcal{M}^{+}$e $y \in \mathcal{M}^{+}$, então $K(y):=C_{2}(y)$ e temos duas possibilidades:

(IV.I) Ou há pelo menos uma colisão com $\Gamma^{0}$ entre $s=0$ e $s_{1}$, daí $K(y):=C_{2}(y)$ e segue de modo análogo ao caso (II.II).

(IV.II) Ou não há colisão com $\Gamma^{0}$ entre $s=0$ e $s_{1}$, assim o pedaço da trajetória é uma corda de comprimento $\tau=\left(2 \cos \alpha_{1}\right) / \mathcal{K}_{f}$, substituindo em (4.3) temos

$$
f_{1}^{+}(v)=\frac{2 \cos \alpha_{1}}{3 \mathcal{K}_{f}}
$$

ou seja,

$$
\frac{\cos \alpha_{1}}{2 \mathcal{K}_{f}} \leq f_{1}^{+}(v) \leq \frac{\cos \alpha_{1}}{\mathcal{K}_{f}}
$$

\subsubsection{Condição L3 - Sinai-Chernov ansatz}

Definição 9. Um ponto $x \in \mathcal{M} \backslash \partial \mathcal{M}$ é chamado u-essencial se para cada $\alpha>0$, existe $n_{x, \alpha} \in \mathbb{N}$, uma vizinhança $O_{x, \alpha}$ de $x$ e um campo de cone invariante $\left(O_{x, \alpha} \cup \mathcal{F}^{n_{x, \alpha}} O_{x, \alpha}, K_{x, \alpha}\right)$ tal que $O_{x, \alpha} \cap$ $\mathcal{R}_{n}^{+}=\emptyset$ e $\sigma_{K_{x, \alpha}^{*}}^{*}\left(D_{y} \mathcal{F}^{n_{x, \alpha}}\right)>\alpha$ para cada $y \in O_{x, \alpha}$. Analogamente, um ponto $x \in \mathcal{M} \backslash \partial \mathcal{M}$ é chamado s-essencial se na definição anterior, $\mathcal{F}$ e $\mathcal{R}_{n}^{+}$são substituídos por $\mathcal{F}^{-1}$ e $\mathcal{R}_{n}^{-}$, respectivamente.

O campo de cone $(O, K)$ na Definição 7 é certamente estritamente invariante. Por um resultado em [Woj85, Mar88] segue que os expoentes de Lyapunov de $\mathcal{F}$ são não nulos quase sempre em $\bigcup_{k \in \mathbb{Z}} \mathcal{F}^{k} O$. Esse fato juntamente com a teoria de Katok-Strelcyn [KS86] nos dá a Proposição abaixo (Parte (3) é provada em [MM13a] Proposição 5.3). Para a definição de continuidade absoluta de uma foleação, nos referimos a [CM06, KS86].

Proposição 5. Seja $x \in \mathcal{M} \backslash \partial \mathcal{M}$ um ponto suficiente com quádrupla $(l, N, K, O)$. Então existe um conjunto invariante $\Lambda_{x} \subset \bigcup_{k \in \mathbb{Z}} \mathcal{F}^{k} O$ com $\mu\left(\left(\bigcup_{k \in \mathbb{Z}} \mathcal{F}^{k} O\right) \backslash \Lambda_{x}\right)=0$ e duas famílias de $C^{2}$ subvariedades invariantes $V^{s}=\left\{V_{y}^{s}\right\}_{y \in \Lambda_{x}} e V^{u}=\left\{V_{y}^{u}\right\}_{y \in \Lambda_{x}}$ tais que para cada $y \in \Lambda_{x}$, tem-se

1. $V_{y}^{s} \cap V_{y}^{u}=\{y\}$,

2. $V_{y}^{s}$ e $V_{y}^{u}$ são difeomorfos a intervalos abertos,

3. $T_{y} V_{y}^{s} \subset K^{\prime}(y)$ e $T_{y} V_{y}^{u} \subset K(y)$ dado que $y \in O \cup \mathcal{F}^{-N} O$,

4. $\mathcal{F} V_{y}^{s} \subset V_{\mathcal{F} y}^{s}$ e $\mathcal{F}^{-1} V_{y}^{u} \subset V_{\mathcal{F}^{-1} y}^{u}$,

5. $d\left(\mathcal{F}^{n} y, \mathcal{F}^{n} z\right) \rightarrow 0$ exponencialmente quando $n \rightarrow+\infty$ para cada $z \in V_{y}^{s}$, e o mesmo é verdade quando $n \rightarrow-\infty$ para cada $z \in V_{y}^{u}$,

6. $V_{y}^{s}$ e $V_{y}^{u}$ variam mensuravelmente com $y \in \Lambda_{x}$,

7. $V^{s}$ e $V^{u}$ tem a propriedade de continuidade absoluta.

Definição 10. As subvariedades formando as famílias $V^{s}$ e $V^{u}$ são chamadas variedades estável local e variedades instável local, respectivamente. 
Definição 11. Seja $x$ um ponto suficiente de $\mathcal{M} \backslash \partial \mathcal{M}$ e seja $\Lambda_{x}$ o conjunto na Proposição 5. Para cada $y \in \Lambda_{x}$, denotamos por $W_{y}^{u}$ a componente conexa de

$$
\bigcup_{k \geq 0} \mathcal{F}^{k} V_{\mathcal{F}^{-k} y}^{u}
$$

contendo y. Analogamente, denotamos por $W_{y}^{s}$ o conjunto obtido por substituir $\mathcal{F}$ com $\mathcal{F}^{-1}$ e $V^{u}$ com $V^{s}$ na definição de $W_{y}^{u}$.

Definição 12. Sejam $C_{1}$ e $C_{2}$ dois campos de cone definidos nos abertos $U_{1}$ e $U_{2}$, respectivamente. Dizemos que $C_{1}$ e $C_{2}$ são invariantes conjunto se:

- $D_{x} \mathcal{F}^{k} C_{1}(x) \subset C_{2}\left(\mathcal{F}^{k} x\right)$ para cada $x \in U_{1}$ e $k>0$ tal que $\mathcal{F}^{k} x \in U_{2}$;

- $D_{x} \mathcal{F}^{k} C_{2}(x) \subset C_{1}\left(\mathcal{F}^{k} x\right)$ para cada $x \in U_{2}$ e $k>0$ tal que $\mathcal{F}^{k} x \in U_{1}$.

Observação 5. Vemos que na definição anterior não pedimos que os abertos $U_{1}$ e $U_{2}$ sejam disjuntos, muito menos que o campo de cones $C_{1}$ e $C_{2}$ sejam invariantes. Contudo, pode-se ver que $C_{1}$ e $C_{2}$ são invariantes no seguinte sentido: se $x \in U_{1}$ e $k_{2}>k_{1}>0$ tal que $\mathcal{F}^{k_{1}} x \in U_{2}$ e $\mathcal{F}^{k_{2}} x \in U_{1}$, então $D_{x} \mathcal{F}^{k_{2}} C_{1}(x) \subset C_{1}\left(\mathcal{F}^{k_{2}} x\right)$. O mesmo é verdade para $C_{2}$, quando $U_{1}$ é substituído por $U_{2}$.

Seja $x \in \mathcal{M} \backslash \partial \mathcal{M}$ um ponto suficiente com quádrupla $(l, N, O, K)$. Aqui $m$ denota a medida volume gerada pela métrica riemanniana $g$.

Definição 13. Dizemos que $x$ satisfaz a Condição L3 se o conjunto dos pontos u-essenciais de $\mathcal{S}_{1}^{-}$(resp. s-essencial de $\mathcal{S}_{1}^{+}$) possui $m_{-}$medida total (resp. $m_{+}$medida), e se y é qualquer desses pontos, então o campo de cone $(O, K)$ e $\left(O_{y, \alpha}, K_{y, \alpha}\right)$ são invariantes conjunto para cada $\alpha>0$.

Proposição 6. Cada ponto $x \in \mathcal{H}$ satisfaz a Condição L3.

Antes de demonstrar a Proposição 6, daremos alguns prévios resultados.

Observação 6. O símbolo " " na prova da próxima proposição tem o seguinte sentido: dadas duas funções $f$ e $g$ de uma variável natural $n$, então $f \sim g$ (quando $n \rightarrow \infty$ ) se, e somente se, $\lim _{n \rightarrow \infty} f(n) / g(n)=1$.

Proposição 7. Suponha que $x \in E_{j} \backslash R_{m}^{+}$para algum $m \geq 1$ tal que $\mathcal{F}^{m} x \in E_{k}$ e $\mathcal{F}^{r} x \notin E$ para cada $1 \leq r<m$. Então existe uma constante $\gamma>1$ tal que

$$
\sigma_{C}\left(D_{x} \mathcal{F}^{m}\right) \geq \gamma
$$

Demonstração. Provemos que em todos os casos possíveis que $\sigma_{C}\left(D_{x} \mathcal{F}^{m}\right) \gg 1$ e, portanto, existe $\gamma>1$ tal que $\sigma_{C}\left(D_{x} \mathcal{F}^{m}\right) \geq \gamma$. Em [Woj86] - Lema A.4 é possível obter uma fórmula para $\sigma_{C}\left(D_{x} \mathcal{F}^{m}\right)$, que é dada por $\sigma_{C}\left(D_{x} \mathcal{F}^{m}\right)=\sqrt{\rho+1}+\sqrt{\rho}$, onde $\rho=1 /(\zeta-1)$ e $\zeta$ é o cross ratio definido em (2.1).

(1) $x \in \mathcal{M}_{1}^{-}, \mathcal{F} x \in \mathcal{M}_{2}^{-}$.

Temos que,

$$
C_{0}(\mathcal{F} x)=\left\{v \in T_{\mathcal{F} x} \mathcal{M}: \frac{\cos \alpha_{1}}{\mathcal{K}_{d}} \leq f^{+}(\mathcal{F} x, v) \leq 0\right\} .
$$

Agora, por invariância estrita, de [BL08] sabemos que $F_{1}^{+} \in D_{-4}^{\circ}(\mathcal{F} x) \subset D_{-2}^{\circ}(\mathcal{F} x)$ e assim, devemos ter $r_{1}$ e $l_{1}$ de $(2.1)$ tais que

$$
D_{x} \mathcal{F} C_{0}(x)=\left\{v \in T_{\mathcal{F} x} \mathcal{M}: \frac{\cos \alpha_{1}}{2 \mathcal{K}_{d}}<l_{1} \leq f^{+}(\mathcal{F} x, v) \leq r_{1}<0\right\} .
$$


cujos valores serão obtidos a frente. Dessa forma, obtemos:

$$
\zeta=\frac{l_{1}\left(\mathcal{K}_{d} r_{1}-\cos \alpha_{1}\right)}{r_{1}\left(\mathcal{K}_{d} l_{1}-\cos \alpha_{1}\right)}
$$

Pelo Lema 1 e usando o fato que $f_{1}^{-}=f_{0}^{+}-\tau$ com $f_{0}^{+}=0\left(\right.$ para $\left.r_{1}\right)$, e $f_{0}^{+}=\left(\cos \alpha_{0}\right) / \mathcal{K}_{d}\left(\right.$ para $\left.l_{1}\right)$ temos

$$
r_{1}=\frac{\tau \cos \alpha_{1}}{2 \tau \mathcal{K}_{d}-\cos \alpha_{1}}, \quad l_{1}=\frac{\cos \alpha_{1}\left(\frac{\cos \alpha_{0}}{\mathcal{K}_{d}}-\tau\right)}{2 \mathcal{K}_{d}\left(\frac{\cos \alpha_{0}}{\mathcal{K}_{d}}-\tau\right)+\cos \alpha_{1}} .
$$

Agora,

$$
0<\zeta-1=\frac{\cos \alpha_{1}\left(r_{1}-l_{1}\right)}{r_{1}\left(\mathcal{K}_{d} l_{1}-\cos \alpha_{1}\right)}
$$

$\operatorname{logo} \rho=\frac{1}{\zeta-1}=\frac{r_{1}\left(\mathcal{K}_{d} l_{1}-\cos \alpha_{1}\right)}{\cos \alpha_{1}\left(r_{1}-l_{1}\right)}$. Vemos que

$$
\frac{1}{\cos \alpha_{1}\left(r_{1}-l_{1}\right)} \geq-\frac{2 \mathcal{K}_{d}}{\cos ^{2} \alpha_{1}} \geq 2 \mathcal{K}_{d}
$$

daí

$$
\rho=\frac{1}{\zeta-1}=\frac{r_{1}\left(\mathcal{K}_{d} l_{1}-\cos \alpha_{1}\right)}{\cos \alpha_{1}\left(r_{1}-l_{1}\right)} \geq 2 \mathcal{K}_{d} r_{1}\left(\mathcal{K}_{d} l_{1}-\cos \alpha_{1}\right) .
$$

Observe que $\left(l_{1} \mathcal{K}_{d}-\cos \alpha_{1}\right) \sim 0$ se, e somente se, $\tau \sim\left(\cos \alpha_{0} / \mathcal{K}_{d}+\cos \alpha_{1} / \mathcal{K}_{d}\right)<0$, um absurdo. Assim, vemos que $\rho \sim 0$ se, e somente se, $\alpha_{1} \sim \pm \pi / 2$ (i.é entradas tangentes) então $\cos \alpha_{1} \sim 0$ e assim $r_{1}, l_{1} \sim 0$. Nesse caso devemos estudar a próxima colisão $\mathcal{F}^{2} x$, em que $\mathcal{F}^{2} x \in \mathcal{M}_{3}^{-}$ou $\mathcal{F}^{2} x \in \mathcal{M}^{+}$.

(1.1) Se $\mathcal{F}^{2} x \in \mathcal{M}_{3}^{-}$então

$$
C_{0}\left(\mathcal{F}^{2} x\right)=\left\{v \in T_{\mathcal{F}^{2} x} \mathcal{M}: \frac{\cos \alpha_{2}}{\mathcal{K}_{d}} \leq f^{+}\left(\mathcal{F}^{2} x, v\right) \leq 0\right\}
$$

e por invariância estrita, deve-se existir $r_{1}^{\prime}$ e $l_{1}^{\prime}$ tais que

$$
D_{\mathcal{F} x} \mathcal{F}\left(D_{x} \mathcal{F} C_{0}(x)\right)=\left\{v \in T_{\mathcal{F}^{2} x} \mathcal{M}: \frac{\cos \alpha_{2}}{2 \mathcal{K}_{d}}<l_{1}^{\prime} \leq f^{+}\left(\mathcal{F}^{2} x, v\right) \leq r_{1}^{\prime}<0\right\}
$$

onde

$$
r_{1}^{\prime}=\frac{\cos \alpha_{2}\left(r_{1}-\tau^{\prime}\right)}{2 \mathcal{K}_{d}\left(r_{1}-\tau^{\prime}\right)+\cos \alpha_{2}}, \quad l_{1}^{\prime}=\frac{\cos \alpha_{2}\left(l_{1}-\tau^{\prime}\right)}{2 \mathcal{K}_{d}\left(l_{1}-\tau^{\prime}\right)+\cos \alpha_{2}},
$$

em que $\tau^{\prime}$ é a distância entre $q(\mathcal{F} x)$ e $q\left(\mathcal{F}^{2} x\right)$. Assim fazendo um novo cálculo para o cross ratio, obtemos

$$
\zeta^{\prime}=\frac{l_{1}^{\prime}\left(\mathcal{K}_{d} r_{1}^{\prime}-\cos \alpha_{2}\right)}{r_{1}^{\prime}\left(\mathcal{K}_{d} l_{1}^{\prime}-\cos \alpha_{2}\right)}
$$

Procedendo da mesma forma que anteriormente, segue que

$$
\rho^{\prime} \geq 2 \mathcal{K}_{d} r_{1}^{\prime}\left(\mathcal{K}_{d} l_{1}^{\prime}-\cos \alpha_{2}\right) .
$$

Portanto, observamos que quando $\alpha_{1} \sim \pm \pi / 2$ tem-se $\alpha_{2} \sim 0$ assim como $r_{1}, l_{1} \sim 0$ daí $r_{1}^{\prime}, l_{1}^{\prime} \sim$ $\frac{\tau^{\prime}}{2 \mathcal{K}_{d} \tau^{\prime}-1} \ll 0$. E ainda, $\left(\mathcal{K}_{d} l_{1}^{\prime}-\cos \alpha_{2}\right) \sim 0$ se, e somente se, $\cos \alpha_{2} \sim \mathcal{K}_{d}\left(\tau^{\prime}-l_{1}\right)<0$, o que é um absurdo. Portanto,

$$
\sigma_{C}\left(D_{x} \mathcal{F}^{2}\right) \geq \sqrt{2 \mathcal{K}_{d} r_{1}^{\prime}\left(\cos \alpha_{2}-\mathcal{K}_{d} l_{1}^{\prime}\right)+1}+\sqrt{2 \mathcal{K}_{d} r_{1}^{\prime}\left(\cos \alpha_{2}-\mathcal{K}_{d} l_{1}^{\prime}\right)} \gg 1
$$


(1.2) Se $\mathcal{F}^{2} x \in \mathcal{M}^{+}$temos

$$
C_{1}\left(\mathcal{F}^{2} x\right)=\left\{v \in T_{\mathcal{F}^{2} x} \mathcal{M}:-\infty<f^{+}\left(\mathcal{F}^{2} x, v\right) \leq 0\right\},
$$

temos por [BL08] que $F_{2}^{+} \in D_{0^{-}}\left(\mathcal{F}^{2} x\right)$, isto é, por invariância estrita existem $r_{1}^{\prime \prime}$ e $l_{1}^{\prime \prime}$ tais que

$$
D_{\mathcal{F} x} \mathcal{F}\left(D_{x} \mathcal{F} C_{0}(x)\right)=\left\{v \in T_{\mathcal{F}^{2} x} \mathcal{M}:-\infty<l_{1}^{\prime \prime} \leq f^{+}\left(\mathcal{F}^{2} x, v\right) \leq r_{1}^{\prime \prime}<0\right\},
$$

onde

$$
r_{1}^{\prime \prime}=\frac{\cos \alpha_{2}\left(r_{1}-\tau^{\prime}\right)}{2 \mathcal{K}_{f}\left(r_{1}-\tau^{\prime}\right)+\cos \alpha_{2}}, \quad l_{1}^{\prime \prime}=\frac{\cos \alpha_{2}\left(l_{1}-\tau^{\prime}\right)}{2 \mathcal{K}_{f}\left(l_{1}-\tau^{\prime}\right)+\cos \alpha_{2}}
$$

aqui

$$
\zeta=\frac{l_{1}^{\prime \prime}}{r_{1}^{\prime \prime}} \Rightarrow 0<\rho^{\prime}=\frac{1}{\zeta-1}=\frac{r_{1}^{\prime \prime}}{l_{1}^{\prime \prime}-r_{1}^{\prime \prime}} .
$$

Como $l_{1}^{\prime \prime}-r_{1}^{\prime \prime}>l_{1}^{\prime \prime}$, obtemos $\rho^{\prime}>r_{1}^{\prime \prime} / l_{1}^{\prime \prime}$. Usando o fato que $\cos \alpha_{2} \sim 1$ e $r_{1}, l_{1} \sim 0$, vemos que $r_{1}^{\prime \prime} / l_{1}^{\prime \prime} \sim 1$, ou seja, $\rho^{\prime}>1$. Vemos que $l_{1}^{\prime \prime} \sim-\infty$ se, e somente se, $\left(2 \mathcal{K}_{f} \tau^{\prime}-1\right) \sim 0$ se, e somente se, $\tau^{\prime} \geq \cos \alpha_{2} /\left(2 \mathcal{K}_{f}\right)$, e isso não ocorre pois $\tau^{\prime}<\cos \alpha_{2} /\left(2 \mathcal{K}_{f}\right)$ pela propriedade (3.1). Portanto,

$$
\sigma_{C}\left(D_{x} \mathcal{F}^{2}\right)>\sqrt{\frac{r_{1}^{\prime \prime}}{l_{1}^{\prime \prime}}+1}+\sqrt{\frac{r_{1}^{\prime \prime}}{l_{1}^{\prime \prime}}} \gg 1 .
$$

(2) $x \in \mathcal{M}^{-}$e $\mathcal{F} x \in \mathcal{M}^{+}$. Aqui há dois sub-casos:

(2.1) Sem colisões com $\Gamma^{0}$ entre $x$ e $\mathcal{F} x$.

Temos,

$$
C_{1}(\mathcal{F} x)=\left\{v \in T_{\mathcal{F} x} \mathcal{M}:-\infty<f^{+}(\mathcal{F} x, v) \leq 0\right\} .
$$

Por [BL08], segue que $F_{1}^{+} \in D_{0^{-}}(\mathcal{F} x), \operatorname{logo}$ existem $r_{1}$ e $l_{1}$ tais que

$$
D_{x} \mathcal{F} C_{0}(x)=\left\{v \in T_{\mathcal{F} x} \mathcal{M}:-\infty<l_{1} \leq f^{+}(\mathcal{F} x, v) \leq r_{1}<0\right\},
$$

onde

$$
r_{1}=\frac{-\tau \cos \alpha_{1}}{-2 \mathcal{K}_{f} \tau+\cos \alpha_{1}}, \quad l_{1}=\frac{\cos \alpha_{1}\left(-\tau+\frac{\cos \alpha_{0}}{\mathcal{K}_{d}}\right)}{\cos \alpha_{1}+2 \mathcal{K}_{f}\left(-\tau+\frac{\cos \alpha_{0}}{\mathcal{K}_{d}}\right)} .
$$

Aqui $\zeta=l_{1} / r_{1} \operatorname{logo} \rho=\frac{1}{\zeta-1}=\frac{r_{1}}{l_{1}-r_{1}}>\frac{r_{1}}{l_{1}}>0$.

Vemos que $r_{1} \ll 0$, pois $\left|\alpha_{1}\right| \ll \pi / 2$ e assim $\cos \alpha_{1} \gg 0$ e, ainda, $l_{1} \sim-\infty$ se, e somente se, $\left(2 \mathcal{K}_{f}\left(-\tau+\cos \alpha / \mathcal{K}_{d}\right)+\cos \alpha_{1}\right) \sim 0$ se, e somente se, $\tau \sim \cos \alpha / \mathcal{K}_{d}+\left(\cos \alpha_{1}\right) / 2 \mathcal{K}_{f}$ o que não ocorre pois pela propriedade $(3.1)$ temos $I(x, \mathcal{F} x) \cap D_{4}(\mathcal{F} x)=\emptyset$. Portanto,

$$
\sigma_{C}\left(D_{x} \mathcal{F}\right)>\sqrt{\frac{r_{1}}{l_{1}}+1}+\sqrt{\frac{r_{1}}{l_{1}}} \gg 1
$$

(2.2) Com pelo menos uma colisão com $\Gamma^{0}$ entre $x$ e $\mathcal{F} x$.

Vemos que nesse caso temos $\tau>2 l>2 / \mathcal{K}_{f}$. Como $F_{1}^{+} \in D_{2}^{\circ}(\mathcal{F} x) \backslash D_{4}(\mathcal{F} x)$, temos

$$
\begin{gathered}
C_{2}(\mathcal{F} x)=\left\{v \in T_{\mathcal{F} x} \mathcal{M}: \frac{\cos \alpha_{1}}{2 \mathcal{K}_{f}} \leq f^{+}(\mathcal{F} x, v) \leq \frac{\cos \alpha_{1}}{\mathcal{K}_{f}}\right\}, \\
D_{x} \mathcal{F} C_{0}(x)=\left\{v \in T_{\mathcal{F} x} \mathcal{M}: \frac{\cos \alpha_{1}}{2 \mathcal{K}_{f}}<l_{1} \leq f^{+}(\mathcal{F} x, v) \leq r_{1}<\frac{\cos \alpha_{1}}{\mathcal{K}_{f}}\right\},
\end{gathered}
$$

onde $r_{1}$ e $l_{1}$ são os mesmos que no caso anterior. 
Um cálculo simples nos dá,

$$
\zeta=\frac{\left(\cos \alpha_{1}-\mathcal{K}_{f} l_{1}\right)\left(2 \mathcal{K}_{f} r_{1}-\cos \alpha_{1}\right)}{\left(\cos \alpha_{1}-\mathcal{K}_{f} r_{1}\right)\left(2 \mathcal{K}_{f} l_{1}-\cos \alpha_{1}\right)}
$$

daí

$$
\zeta-1=\frac{\mathcal{K}_{f} \cos \alpha_{1}\left(r_{1}-l_{1}\right)}{\left(\cos \alpha_{1}-\mathcal{K}_{f} r_{1}\right)\left(2 \mathcal{K}_{f} l_{1}-\cos \alpha_{1}\right)}>0
$$

Pela definição do cone $D_{x} \mathcal{F} C_{0}(x)$, segue que $r_{1}-l_{1}<\frac{1}{2 \mathcal{K}_{f}}$ o que implica $1 /\left(\mathcal{K}_{f} \cos \alpha_{1}\left(r_{1}-l_{1}\right)\right)>2$, assim

$$
\rho=\frac{1}{\zeta-1}=\frac{\left(\cos \alpha_{1}-\mathcal{K}_{f} r_{1}\right)\left(2 \mathcal{K}_{f} l_{1}-\cos \alpha_{1}\right)}{\mathcal{K}_{f} \cos \alpha_{1}\left(r_{1}-l_{1}\right)}>2\left(\cos \alpha_{1}-\mathcal{K}_{f} r_{1}\right)\left(2 \mathcal{K}_{f} l_{1}-\cos \alpha_{1}\right),
$$

ou seja,

$$
\rho>\left(\cos \alpha_{1}-\mathcal{K}_{f} r_{1}\right)\left(2 \mathcal{K}_{f} l_{1}-\cos \alpha_{1}\right) .
$$

Temos $\left(\cos \alpha_{1}-\mathcal{K}_{f} r_{1}\right) \sim 0$ se, e somente se, $\tau \sim \cos \alpha_{1} / \mathcal{K}_{f} \leq 1 / \mathcal{K}_{f}$, o que é um absurdo pois $\tau>2 / \mathcal{K}_{f}$. Se $\left|\alpha_{1}\right|>\pi / 2-\epsilon$, com $\epsilon$ fixado, suficientemente pequeno, a trajetória irá novamente para a parte flat. Assim, $x, \mathcal{F} x \in \mathcal{M}^{+}$e $\mathcal{F}^{n} x \in \mathcal{M}^{+}$para $1 \leq n \leq n(x)$, e também, $\mathcal{F}^{m} x \in \mathcal{M}^{0}$, para $n(x)<m<m(x)$ e, ainda, $\mathcal{F}^{m(x)} \in E \subset \mathcal{M}^{+} \cup \mathcal{M}^{-}$. Dessa forma, nosso objetivo consiste em mostrar que $\sigma\left(D_{x} \mathcal{F}^{m(x)}\right) \gg 1$. Sabemos

$$
\sigma_{C}\left(D_{x} \mathcal{F}^{n(x)}\right)>\sqrt{\left(\cos \alpha_{1}-\mathcal{K}_{f} r_{1}\right)\left(2 \mathcal{K}_{f} l_{1}-\cos \alpha_{1}\right)+1}+\sqrt{\left(\cos \alpha_{1}-\mathcal{K}_{f} r_{1}\right)\left(2 \mathcal{K}_{f} l_{1}-\cos \alpha_{1}\right)} \gg 1,
$$

porém na parte flat temos $\sigma\left(D_{x} \mathcal{F}^{m(x)}\right) \geq \sigma\left(D_{x} \mathcal{F}^{n(x)}\right) \gg 1$, para $\mathcal{F}^{m(x)} \in E$.

(3) $x \in \mathcal{M}^{+}, \mathcal{F} x \in \mathcal{M}^{-}$. Aqui há dois sub-casos:

(3.1) Sem colisões com $\Gamma^{0}$ entre $x$ e $\mathcal{F} x$.

Primeiramente, vamos estudar o caso em que $C(x)=C_{1}(x)$. Daí,

$$
C_{0}(\mathcal{F} x)=\left\{v \in T_{\mathcal{F} x} \mathcal{M}: \frac{\cos \alpha_{1}}{\mathcal{K}_{d}} \leq f^{+}(\mathcal{F} x, v) \leq 0\right\} .
$$

Por invariância estrita, de [BL08] sabemos que $F_{1}^{+} \in D_{-4}^{\circ}(\mathcal{F} x) \subset D_{-2}^{\circ}(\mathcal{F} x)$, assim existem $r_{1}$ e $l_{1}$ tais que

$$
D_{x} \mathcal{F} C_{1}(x)=\left\{v \in T_{\mathcal{F} x} \mathcal{M}: \frac{\cos \alpha_{1}}{2 \mathcal{K}_{d}}<l_{1} \leq f^{+}(\mathcal{F} x, v) \leq r_{1}<0\right\}
$$

onde

$$
r_{1}=\frac{\tau \cos \alpha_{1}}{2 \tau \mathcal{K}_{d}-\cos \alpha_{1}}, \quad l_{1}=\frac{\cos \alpha_{1}(\bar{c}-\tau)}{2 \mathcal{K}_{d}(\bar{c}-\tau)+\cos \alpha_{1}},
$$

com $-\infty<\bar{c}<0$. Assim, o cross ratio é dado por

$$
\zeta=\frac{l_{1}\left(\mathcal{K}_{d} r_{1}-\cos \alpha_{1}\right)}{r_{1}\left(\mathcal{K}_{d} l_{1}-\cos \alpha_{1}\right)}
$$

e portanto,

$$
\rho \geq 2 \mathcal{K}_{d} r_{1}\left(\cos \alpha_{1}-\mathcal{K}_{d} l_{1}\right) .
$$

Vemos que $\left(\cos \alpha_{1}-\mathcal{K}_{d} l_{1}\right) \sim 0$ se, e somente se, $\tau \sim\left(\bar{c}+\cos \alpha_{1} / \mathcal{K}_{d}\right)<0$ um absurdo. Porém, 
pode ocorrer $r_{1} \sim 0$, isto é, quando $\alpha_{1} \sim \pm \pi / 2$ (entradas tangentes) e então $\cos \alpha_{1} \sim 0$. Assim, estudando a próxima colisão $\mathcal{F}^{2} x \in \mathcal{M}_{2}^{-}$temos $F_{2}^{+} \in D_{-4}^{\circ}\left(\mathcal{F}^{2} x\right)$ e, portanto,

$$
D_{\mathcal{F} x} \mathcal{F}\left(D_{x} \mathcal{F} C_{1}(x)\right)=\left\{v \in T_{\mathcal{F}^{2} x} \mathcal{M}: \frac{\cos \alpha_{2}}{2 \mathcal{K}_{d}}<l_{1}^{\prime} \leq f^{+}\left(\mathcal{F}^{2} x, v\right) \leq r_{1}^{\prime}<0\right\},
$$

onde

$$
r_{1}^{\prime}=\frac{\cos \alpha_{2}\left(r_{1}-\tau^{\prime}\right)}{2 \mathcal{K}_{d}\left(r_{1}-\tau^{\prime}\right)+\cos \alpha_{2}}, \quad l_{1}^{\prime}=\frac{\cos \alpha_{2}\left(l_{1}-\tau^{\prime}\right)}{2 \mathcal{K}_{d}\left(l_{1}-\tau^{\prime}\right)+\cos \alpha_{2}} .
$$

Prosseguindo como antes, obtemos

$$
\zeta^{\prime}=\frac{l_{1}^{\prime}\left(\mathcal{K}_{d} r_{1}^{\prime}-\cos \alpha_{2}\right)}{r_{1}^{\prime}\left(\mathcal{K}_{d} l_{1}^{\prime}-\cos \alpha_{2}\right)}
$$

e

$$
\rho^{\prime} \geq 2 \mathcal{K}_{d} r_{1}^{\prime}\left(\cos \alpha_{2}-\mathcal{K}_{d} l_{1}^{\prime}\right)
$$

Como $\alpha_{1} \sim \pm \pi / 2$ temos $\alpha_{2} \sim 0$ e assim $r_{1}^{\prime} \ll 0$. E ainda, $\left(\cos \alpha_{2}-\mathcal{K}_{d} l_{1}^{\prime}\right) \sim 0$ se, e somente se, $\tau^{\prime} \sim\left(l_{1}+\cos \alpha_{2} / \mathcal{K}_{d}\right)<0$, o que é um absurdo. Portanto,

$$
\sigma_{C}\left(D_{x} \mathcal{F}^{2}\right) \geq \sqrt{2 \mathcal{K}_{d} r_{1}^{\prime}\left(\cos \alpha_{2}-\mathcal{K}_{d} l_{1}^{\prime}\right)+1}+\sqrt{2 \mathcal{K}_{d} r_{1}^{\prime}\left(\cos \alpha_{2}-\mathcal{K}_{d} l_{1}^{\prime}\right)} \gg 1 .
$$

Para o caso $C(x)=C_{2}(x)$, segue por invariância estrita que $F_{1}^{+} \in D_{-2}(\mathcal{F} x) \backslash D_{-4}^{\circ}(\mathcal{F} x)$, assim

$$
\begin{gathered}
C_{0}(\mathcal{F} x)=\left\{v \in T_{\mathcal{F} x} \mathcal{M}: \frac{\cos \alpha_{1}}{\mathcal{K}_{d}} \leq f^{+}(\mathcal{F} x, v) \leq 0\right\} \\
D_{x} \mathcal{F} C_{2}(x)=\left\{v \in T_{\mathcal{F} x} \mathcal{M}: \frac{\cos \alpha_{1}}{\mathcal{K}_{d}}<l_{1} \leq f^{+}(\mathcal{F} x, v) \leq r_{1} \leq 0\right\} .
\end{gathered}
$$

onde

$$
r_{1}=\frac{\cos \alpha_{1}\left(-\tau+\frac{\cos \alpha_{0}}{\mathcal{K}_{f}}\right)}{\cos \alpha_{1}+2 \mathcal{K}_{d}\left(-\tau+\frac{\cos \alpha_{0}}{\mathcal{K}_{f}}\right)}, \quad l_{1}=\frac{\cos \alpha_{1}\left(-\tau+\frac{\cos \alpha_{0}}{2 \mathcal{K}_{f}}\right)}{\cos \alpha_{1}+2 \mathcal{K}_{d}\left(-\tau+\frac{\cos \alpha_{0}}{2 \mathcal{K}_{f}}\right)} .
$$

Fazendo o cálculo do cross ratio, obtemos

$$
\zeta=\frac{l_{1}\left(\mathcal{K}_{d} r_{1}-\cos \alpha_{1}\right)}{r_{1}\left(\mathcal{K}_{d} l_{1}-\cos \alpha_{1}\right)}
$$

daí

$$
\rho=\frac{1}{\zeta-1}=\frac{r_{1}\left(\mathcal{K}_{d} l_{1}-\cos \alpha_{1}\right)}{\cos \alpha_{1}\left(r_{1}-l_{1}\right)} .
$$

Vemos que $0<r_{1}-l_{1}<-l_{1}<-\cos \alpha_{1} / \mathcal{K}_{d}$, logo $\cos \alpha_{1}\left(r_{1}-l_{1}\right)<-\cos ^{2} \alpha_{1} / \mathcal{K}_{d}$ daí

$$
\frac{1}{\cos \alpha_{1}\left(r_{1}-l_{1}\right)}>\frac{-\mathcal{K}_{d}}{\cos ^{2} \alpha_{1}}
$$

isso implica que

$$
\rho=\frac{r_{1}\left(\mathcal{K}_{d} l_{1}-\cos \alpha_{1}\right)}{\cos \alpha_{1}\left(r_{1}-l_{1}\right)}>\mathcal{K}_{d} r_{1}\left(\cos \alpha_{1}-\mathcal{K}_{d} l_{1}\right)
$$

Aqui $r_{1} \sim 0$ se, e somente se, $\alpha_{1} \sim \pm \pi / 2$ (e assim devemos estudar $\mathcal{F}^{2} x \in \mathcal{M}^{-}$que cai no caso (1)) ou $-\tau+\cos \alpha_{0} / \mathcal{K}_{f} \sim 0$, isto é, $\tau \sim \cos \alpha_{0} / \mathcal{K}_{f}$ que também não ocorre, pois aqui 
$\tau<\cos \left(\alpha_{0}\right) / 2 \mathcal{K}_{f}$. E ainda, $\cos \alpha_{1}-\mathcal{K}_{d} l_{1} \sim 0$ se, e somente se, $\tau \sim \cos \left(\alpha_{0}\right) / 2 \mathcal{K}_{f}+\cos \alpha_{1} / \mathcal{K}_{d}$, o que é um absurdo, já que a propriedade $(3.1)$ nos garante que $D_{4}(x) \cap I(x, \mathcal{F} x)=\emptyset$. Portanto,

$$
\sigma_{C}\left(D_{x} \mathcal{F}^{2}\right)>\sqrt{\mathcal{K}_{d} r_{1}\left(\cos \alpha_{1}-\mathcal{K}_{d} l_{1}\right)+1}+\sqrt{\mathcal{K}_{d} r_{1}\left(\cos \alpha_{1}-\mathcal{K}_{d} l_{1}\right)} \gg 1
$$

(3.2) Com pelo menos uma colisão com $\Gamma^{0}$ entre $x$ e $\mathcal{F} x$.

Se $C(x)=C_{1}(x)$ tem-se:

$$
\begin{gathered}
C_{0}(\mathcal{F} x)=\left\{v \in T_{\mathcal{F} x} \mathcal{M}: \frac{\cos \alpha_{1}}{\mathcal{K}_{d}} \leq f^{+}(\mathcal{F} x, v) \leq 0\right\}, \\
D_{x} \mathcal{F} C_{1}(x)=\left\{v \in T_{\mathcal{F} x} \mathcal{M}: \frac{\cos \alpha_{1}}{\mathcal{K}_{d}}<l_{1} \leq f^{+}(\mathcal{F} x, v) \leq r_{1}<0\right\},
\end{gathered}
$$

onde

$$
r_{1}=\frac{\tau \cos \alpha_{1}}{2 \tau \mathcal{K}_{d}-\cos \alpha_{1}}, \quad l_{1}=\frac{\cos \alpha_{1}(\bar{c}-\tau)}{2 \mathcal{K}_{d}(\bar{c}-\tau)+\cos \alpha_{1}},
$$

são obtidos por invariância estrita como anteriormente e $-\infty<\bar{c}<0$. Assim,

$$
\zeta=\frac{l_{1}\left(\mathcal{K}_{d} r_{1}-\cos \alpha_{1}\right)}{r_{1}\left(\mathcal{K}_{d} l_{1}-\cos \alpha_{1}\right)}
$$

e portanto,

$$
\rho \geq 2 \mathcal{K}_{d} r_{1}\left(\cos \alpha_{1}-\mathcal{K}_{d} l_{1}\right) .
$$

Vemos que o fato de ter colisões com $\Gamma^{0}$ nos dá que $r_{1} \ll 0$, já que $\tau \gg 0$ e $\left|\alpha_{1}\right| \ll \pm \pi / 2$. Vemos também que $\cos \alpha_{1}-\mathcal{K}_{d} l_{1} \sim 0$ se, e somente se, $\tau \sim \bar{c}+\cos \alpha_{1} / \mathcal{K}_{d}<0$, um absurdo. Logo,

$$
\sigma_{C}\left(D_{x} \mathcal{F}\right)>\sqrt{2 \mathcal{K}_{d} r_{1}\left(\cos \alpha_{1}-\mathcal{K}_{d} l_{1}\right)+1}+\sqrt{2 \mathcal{K}_{d} r_{1}\left(\cos \alpha_{1}-\mathcal{K}_{d} l_{1}\right)} \gg 1 .
$$

Agora, para o caso $C(x)=C_{2}(x)$ recorde que $\tau>2 / \mathcal{K}_{f}$ e $F_{1}^{+} \in D_{-4}^{\circ}(\mathcal{F} x) \subset D_{-2}^{\circ}(\mathcal{F} x)$, assim

$$
\begin{gathered}
C_{0}(\mathcal{F} x)=\left\{v \in T_{\mathcal{F} x} \mathcal{M}: \frac{\cos \alpha_{1}}{\mathcal{K}_{d}} \leq f^{+}(\mathcal{F} x, v) \leq 0\right\} \\
D_{x} \mathcal{F} C_{2}(x)=\left\{v \in T_{\mathcal{F} x} \mathcal{M}: \frac{\cos \alpha_{1}}{\mathcal{K}_{d}}<l_{1} \leq f^{+}(\mathcal{F} x, v) \leq r_{1}<0\right\} .
\end{gathered}
$$

onde

$$
r_{1}=\frac{\cos \alpha_{1}\left(-\tau+\frac{\cos \alpha_{0}}{\mathcal{K}_{f}}\right)}{\cos \alpha_{1}+2 \mathcal{K}_{d}\left(-\tau+\frac{\cos \alpha_{0}}{\mathcal{K}_{f}}\right)}, \quad l_{1}=\frac{\cos \alpha_{1}\left(-\tau+\frac{\cos \alpha_{0}}{2 \mathcal{K}_{f}}\right)}{\cos \alpha_{1}+2 \mathcal{K}_{d}\left(-\tau+\frac{\cos \alpha_{0}}{2 \mathcal{K}_{f}}\right)}
$$

são os valores obtidos por invariância estrita. Seguindo o caso (3.1) temos

$$
\rho>\mathcal{K}_{d} r_{1}\left(\cos \alpha_{1}-\mathcal{K}_{d} l_{1}\right) .
$$

Agora, $r_{1} \sim 0$ se, e somente se, $\alpha_{1} \sim \pm \pi / 2$ o que não ocorre (já que saídas das faixas não produzem tangências com componentes dispersoras), ou $-\tau+\cos \alpha_{0} / \mathcal{K}_{f} \sim 0$ se, e somente se, $\tau \sim \cos \alpha_{0} / \mathcal{K}_{f}$, um absurdo já que $\tau>2 / \mathcal{K}_{f}$. E ainda, $\cos \alpha_{1}-\mathcal{K}_{d} l_{1} \sim 0$ se, e somente se, $\tau \sim\left(\cos \alpha_{0}\right) / 2 \mathcal{K}_{f}+$ $\cos \alpha_{1} / \mathcal{K}_{d}<2 / \mathcal{K}_{f}$ também absurdo. Portanto,

$$
\sigma_{C}\left(D_{x} \mathcal{F}\right)>\sqrt{\mathcal{K}_{d} r_{1}\left(\cos \alpha_{1}-\mathcal{K}_{d} l_{1}\right)+1}+\sqrt{\mathcal{K}_{d} r_{1}\left(\cos \alpha_{1}-\mathcal{K}_{d} l_{1}\right)} \gg 1 .
$$

(4) $x, \mathcal{F} x \in \mathcal{M}^{+}$. E assim temos dois sub-casos: 
(4.1) Sem colisões com $\Gamma_{0}$ entre $x$ e $\mathcal{F} x$.

Aqui iremos considerar somente o caso $C(x)=C_{1}(x)$ (ver Observação 7). Vemos que o pedaço da trajetória entre $x$ e $\mathcal{F} x$ é uma corda de comprimento $\tau=(2 \cos \alpha) / \mathcal{K}_{f}$. Como $\mathcal{F} x \in \mathcal{M}^{+}$, temos

$$
C_{2}(\mathcal{F} x)=\left\{v \in T_{\mathcal{F} x} \mathcal{M}: \frac{\cos \alpha_{1}}{2 \mathcal{K}_{f}} \leq f^{+}(\mathcal{F} x, v) \leq \frac{\cos \alpha_{1}}{\mathcal{K}_{f}}\right\} .
$$

Seguindo [BL08], tem-se $F_{1}^{+} \in D_{2}^{\circ}(\mathcal{F} x) \backslash D_{4}(\mathcal{F} x)$, ou seja, por invariância estrita existem $r_{1}$ e $l_{1}$ tais que

$$
D_{x} \mathcal{F} C_{1}(x)=\left\{v \in T_{\mathcal{F} x} \mathcal{M}: \frac{\cos \alpha_{1}}{2 \mathcal{K}_{f}}<l_{1} \leq f^{+}(\mathcal{F} x, v) \leq r_{1}<\frac{\cos \alpha_{1}}{\mathcal{K}_{f}}\right\} .
$$

Com isso, obtemos:

$$
\zeta=\frac{\left(\cos \alpha_{1}-\mathcal{K}_{f} l_{1}\right)\left(2 \mathcal{K}_{f} r_{1}-\cos \alpha_{1}\right)}{\left(\cos \alpha_{1}-\mathcal{K}_{f} r_{1}\right)\left(2 \mathcal{K}_{f} l_{1}-\cos \alpha_{1}\right)}
$$

e assim,

$$
\rho>\left(\cos \alpha_{1}-\mathcal{K}_{f} r_{1}\right)\left(2 \mathcal{K}_{f} l_{1}-\cos \alpha_{1}\right)
$$

Aqui

$$
r_{1}=\frac{-\tau \cos \alpha_{1}}{\cos \alpha_{1}-2 \tau \mathcal{K}_{f}}, \quad l_{1}=\frac{\cos \alpha_{1}(\bar{c}-\tau)}{2 \mathcal{K}_{f}(\bar{c}-\tau)+\cos \alpha_{1}}
$$

com $-\infty<\bar{c}<0$.

Agora, $\cos \alpha_{1} \sim 0$ não ocorre pois estamos entrando na focalizadora por uma componente dispersora. E ainda, $\cos \alpha_{1}-\mathcal{K}_{f} r_{1} \sim 0$ se, e somente se, $\tau \sim \cos \alpha_{1} / \mathcal{K}_{f}$ o que é um absurdo, pois $\tau=\left(2 \cos \alpha_{1}\right) / \mathcal{K}_{f}$. E também, $2 \mathcal{K}_{f} l_{1}-\cos \alpha_{1} \sim 0$ se, e somente se, $\cos \alpha_{1} \sim 0$ o que não ocorre como acabamos de mencionar. Portanto,

$$
\sigma_{C}\left(D_{x} \mathcal{F}\right)>\sqrt{\left(\cos \alpha_{1}-\mathcal{K}_{f} r_{1}\right)\left(2 \mathcal{K}_{f} l_{1}-\cos \alpha_{1}\right)+1}+\sqrt{\left(\cos \alpha_{1}-\mathcal{K}_{f} r_{1}\right)\left(2 \mathcal{K}_{f} l_{1}-\cos \alpha_{1}\right)} \gg 1
$$

(4.2) Com pelo menos uma colisão com $\Gamma_{0}$ entre $x$ e $\mathcal{F} x$.

Se $C(x)=C_{1}(x)$ então os valores $r_{1}$ e $l_{1}$ são os mesmos obtidos em (4.1). O fato de haver colisões com $\Gamma_{0}$, as saídas da faixa poderia ter ângulo próximo de $\pi / 2$. Porém, se $\left|\alpha_{1}\right|>\pi / 2-\epsilon$, com $\epsilon$ fixado, suficientemente pequeno, a trajetória irá novamente para a parte flat. Assim, $x, \mathcal{F} x \in \mathcal{M}^{+}$ e $\mathcal{F}^{n} x \in \mathcal{M}^{+}$para $1 \leq n \leq n(x)$, e também, $\mathcal{F}^{m} x \in \mathcal{M}^{0}$, para $n(x)<m<m(x)$ e, ainda, $\mathcal{F}^{m(x)} \in E \subset \mathcal{M}^{+} \cup \mathcal{M}^{-}$. Dessa forma, nosso objetivo consiste em mostrar que $\sigma\left(D_{x} \mathcal{F}^{m(x)}\right) \gg 1$. Sabemos pelo item anterior que

$$
\sigma_{C}\left(D_{x} \mathcal{F}^{n(x)}\right)>\sqrt{\left(\cos \alpha_{1}-\mathcal{K}_{f} r_{1}\right)\left(2 \mathcal{K}_{f} l_{1}-\cos \alpha_{1}\right)+1}+\sqrt{\left(\cos \alpha_{1}-\mathcal{K}_{f} r_{1}\right)\left(2 \mathcal{K}_{f} l_{1}-\cos \alpha_{1}\right)} \gg 1 .
$$

Porém na parte flat temos $\sigma\left(D_{x} \mathcal{F}^{m(x)}\right) \geq \sigma\left(D_{x} \mathcal{F}^{n(x)}\right) \gg 1$, para $\mathcal{F}^{m(x)} \in E$, o que conclui a demonstração.

Se $C(x)=C_{2}(x)$ temos por invariância que $F_{1}^{+} \in D_{2}^{\circ}(\mathcal{F} x) \backslash D_{4}(\mathcal{F} x)$, e assim existem $r_{1}^{\prime}$ e $l_{1}^{\prime}$ tais que

$$
D_{x} \mathcal{F} C_{2}(x)=\left\{v \in T_{\mathcal{F} x} \mathcal{M}: \frac{\cos \alpha_{1}}{2 \mathcal{K}_{f}}<l_{1}^{\prime} \leq f^{+}(\mathcal{F} x, v) \leq r_{1}^{\prime}<\frac{\cos \alpha_{1}}{\mathcal{K}_{f}}\right\}
$$

Novamente,

$$
\rho>\left(\cos \alpha_{1}-\mathcal{K}_{f} r_{1}^{\prime}\right)\left(2 \mathcal{K}_{f} l_{1}^{\prime}-\cos \alpha_{1}\right)
$$


onde

$$
r_{1}^{\prime}=\frac{\cos \alpha_{1}\left(-\tau+\frac{\cos \alpha_{0}}{\mathcal{K}_{f}}\right)}{\cos \alpha_{1}+2 \mathcal{K}_{f}\left(-\tau+\frac{\cos \alpha_{0}}{\mathcal{K}_{f}}\right)}, \quad l_{1}^{\prime}=\frac{\cos \alpha_{1}\left(-\tau+\frac{\cos \alpha_{0}}{2 \mathcal{K}_{f}}\right)}{\cos \alpha_{1}+2 \mathcal{K}_{f}\left(-\tau+\frac{\cos \alpha_{0}}{2 \mathcal{K}_{f}}\right)} .
$$

Também, $\left(\cos \alpha_{0} / \mathcal{K}_{f}\right) \ll \tau$ e $\left(\cos \alpha_{0}\right) / 2 \mathcal{K}_{f} \ll \tau$ uma vez que aqui $\tau>2 / \mathcal{K}_{f}$. Observe também que $\left(\cos \alpha_{1}-\mathcal{K}_{f} r_{1}^{\prime}\right) \sim 0$ se, e somente se, $\tau \sim\left(\cos \alpha_{1} / \mathcal{K}_{f}+\cos \alpha_{0} / \mathcal{K}_{f}\right) \leq 2 / \mathcal{K}_{f}$ o que não ocorre. Portanto,

$$
\sigma_{C}\left(D_{x} \mathcal{F}^{n(x)}\right)>\sqrt{\left(\cos \alpha_{1}-\mathcal{K}_{f} r_{1}^{\prime}\right)\left(2 \mathcal{K}_{f} l_{1}^{\prime}-\cos \alpha_{1}\right)+1}+\sqrt{\left(\cos \alpha_{1}-\mathcal{K}_{f} r_{1}^{\prime}\right)\left(2 \mathcal{K}_{f} l_{1}^{\prime}-\cos \alpha_{1}\right)} \gg 1,
$$

o que implica na parte flat em $\sigma\left(D_{x} \mathcal{F}^{m(x)}\right) \geq \sigma_{C}\left(D_{x} \mathcal{F}^{n(x)}\right) \gg 1$, para $\mathcal{F}^{m(x)} \in E$

Observação 7. Vemos que no item (4.1) da prova anterior, se $C(x)=C_{2}(x)$ poderá não haver crescimento de $\sigma_{C}$. E esse caso não é estudado na Proposição 7, uma vez que há um número limitado de colisões consecutivas com $\mathcal{M}^{+}$.

Proposição 8. Seja $x \in E \backslash R_{\infty}^{+}$e suponha que exista uma sequência estritamente crescente (decrescente) de inteiros positivos (negativos) $\left\{n_{k}\right\}_{k \in \mathbb{N}}$ tal que $\mathcal{F}^{n_{k}} x \in E$ para cada $k>0$. Então

$$
\lim _{k \rightarrow \infty} \sigma_{C}\left(D_{x} \mathcal{F}^{n_{k}}\right)=\infty
$$

Demonstração. Provemos a proposição somente para o caso em que $\left\{n_{k}\right\}_{k \in \mathbb{N}}$ é uma sequência estritamente crescente de inteiros positivos. Para o outro caso, a prova é semelhante usando o fato que $\sigma_{C}\left(D_{x} \mathcal{F}^{-n}\right)=\sigma_{C}\left(D_{\mathcal{F}^{-n} x} \mathcal{F}^{n}\right)$ para $n>0$ (veja [LW86], Seção 6).

Sem perda de generalidade, podemos assumir que $\mathcal{F}^{j} x \notin E$ para todo $n_{k}<j<n_{k+1}$. Seja $n_{0}=0$ e defina $x_{k}=\mathcal{F}^{n_{k}} x$ e $m_{k}=n_{k+1}-n_{k}$ para $k \geq 0$. Também, seja $\tau_{k}$ a distância entre $q\left(\mathcal{F}^{-1} x_{k+1}\right)$ e $q\left(x_{k+1}\right)$. Pela a supermultiplicatividade de $\sigma$ (veja Lema A.2 de [Woj86]), tem-se

$$
\sigma_{C}\left(D_{x} \mathcal{F}^{n_{k}}\right) \geq \prod_{i=0}^{k-1} \sigma_{C}\left(D_{x_{i}} \mathcal{F}^{m_{i}}\right) .
$$

Pela Proposição 7, vemos que a sequência $\prod_{i=0}^{k-1} \sigma_{C}\left(D_{x_{i}} \mathcal{F}^{m_{i}}\right)$ é estritamente crescente em $k$ e, portanto, divergente. Logo, $\lim _{k \rightarrow \infty} \prod_{i=0}^{k-1} \sigma_{C}\left(D_{x_{i}} \mathcal{F}^{m_{i}}\right)=+\infty$ e segue o resultado.

Corolário 3. Suponha que $x \in \mathcal{H}$. Então existe $l \in \mathbb{Z}$ tal que $\mathcal{F}^{l} x$ é essencial, e então $x$ é suficiente. Se além disso assumimos que $x$ pertence a $\mathcal{S}_{1}^{-} \cup \mathcal{S}_{1}^{+} \cup E$, então $x$ é essencial.

Demonstração. Seja $x \in \mathcal{H}$. Pela definição de $\mathcal{H}$, podemos encontrar uma sequência $n_{k} \nearrow \infty$ tal que ou $\mathcal{F}^{n_{k}} x \in E$ ou $\mathcal{F}^{-n_{k}} \in E$ para $k>0$. Provaremos o corolário somente para o caso $\mathcal{F}^{n_{k}} x \in E$ para $k>0$, a prova para o outro caso é similar.

Seja $x_{k}=\mathcal{F}^{n_{k}} x$ para $k>0$. Pelo Teorema 3, $C$ é certamente estritamente invariante, logo $\sigma_{C}\left(D_{x_{1}} \mathcal{F}^{n_{2}-n_{1}}\right)>1$, e então $\sigma_{C}^{*}\left(D_{x_{1}} \mathcal{F}^{n_{2}-n_{1}}\right)>0$ por (2.3). Fixe $\alpha>0$. A Proposição 8 nos permite encontrar $k>2$ tal que $\sigma_{C}\left(D_{x_{1}} \mathcal{F}^{n_{k}-n_{1}}\right)>\alpha / \sigma_{C}^{*}\left(D_{x_{1}} \mathcal{F}^{n_{2}-n_{1}}\right)$. Usando (2.4), obtemos $\sigma_{C}^{*}\left(D_{x_{1}} \mathcal{F}^{n_{k}-n_{2}}\right)>\alpha$. Agora, não é difícil ver que existe uma vizinhança $V$ de $x_{1}$ tal que $V \subset U_{x_{1}}$, $V \cap \mathcal{R}_{n_{k}-n_{1}}^{+}=\emptyset$ e $\mathcal{F}^{n_{k}-n_{1}} V \subset U_{x_{k}}$. Como $\sigma_{C}^{*}\left(D_{y} \mathcal{F}^{n_{k}-n_{2}}\right)$ é contínua em $y$, podemos escolher $V$ tal que $\sigma_{C}^{*}\left(D_{z} \mathcal{F}^{n_{k}-n_{2}}\right)>\alpha$ para cada $z \in V$. Mas isso significa que $x_{1}$ é u-essencial com $n_{x_{1}, \alpha}=n_{k}-n_{1}$, $O_{x_{1}, \alpha}=V$ e o campo de cone $K_{x_{1}, \alpha}$ é dado por $K_{x_{1}, \alpha}=C_{x_{1}}$ em $V$ e $K_{x_{1}, \alpha}=C_{x_{k}}$ em $\mathcal{F}^{n_{k}-n_{1}} V$. Escolhendo $\alpha=3$, vemos que $x$ é suficiente com quádrupla $(l, N, O, K)$ tal que $l=n_{1}, N=n_{x_{1}, 3}$, $O=\mathcal{F}^{n_{x_{1}}, 3} O_{x_{1}, 3}$ e $K=C_{x_{1}, 3}$.

Para provar a última parte do corolário, observamos que se assumirmos que $x \in \mathcal{S}_{1}^{-} \cup \mathcal{S}_{1}^{+} \cup E$, então $\left(U_{x}, C_{x}\right)$ está definido, (se $x \in \mathcal{H} \cap \mathcal{S}_{1}^{ \pm}$, então $\left.x \in \mathcal{S}_{1}^{ \pm} \backslash \mathcal{R}_{\infty}^{\mp}\right)$ e o argumento anterior pode ser repetido com $x_{1}$ e $n_{1}$ substituindo-os por $x$ e 0 , respectivamente. 
Demonstração da Proposição 6. É suficiente provarmos o resultado somente para $\mathcal{S}_{1}^{-}$, uma vez que para $\mathcal{S}_{1}^{+}$basta trocarmos os símbolos + e - e substituirmos $\mathcal{F}$ com $\mathcal{F}^{-1}$.

Em [MM13b] (Proposições 6.17-6.19) mostra-se que o conjunto $\mathcal{S}_{1}^{-} \cap \mathcal{R}_{\infty}^{+}$é no máximo enumerável, isso implica que $m_{-}\left(\mathcal{S}_{1}^{-} \cap \mathcal{R}_{\infty}^{+}\right)=0$. Portanto, é suficiente provar que cada elemento de $\mathcal{S}_{1}^{-} \backslash \mathcal{R}_{\infty}^{+}$ é u-essencial, e esse fato é consequência do Corolário 3. A segunda parte de L3 é consequência direta da definição do campo de cone $C$ e do fato que ele é certamente estritamente invariante.

\subsubsection{Condição L4 - Contração}

Aqui provamos a Condição L4. Para isso precisamos da existência quase sempre de variedades estáveis e instáveis na vizinhança $O$ de $x$, a qual é garantida pela Proposição 5 .

Definição 14. Dados $x \in \mathcal{M} \backslash \partial \mathcal{M}$ um ponto suficiente com quádrupla $(l, N, O, K)$ e $\Lambda_{x}$ o subconjunto associado à $x$ como na Proposiçãa 5, dizemos que $x$ satisfaz a Condição L4 se existem $\beta>0$ $e \epsilon>0$ tais que

$$
\left\|\left.D_{z} \mathcal{F}^{-k}\right|_{T_{z} W_{y}^{u}}\right\| \leq \beta \quad\left(\text { resp. } \quad\left\|\left.D_{z} \mathcal{F}^{k}\right|_{T z W_{y}^{s}}\right\| \leq \beta\right)
$$

para cada $y \in O \cap \Lambda_{x}$ e cada $z \in O \cap W_{y}^{u} \cap \mathcal{F}^{k} \mathcal{S}_{1}^{-}(\epsilon) \quad$ (resp. $\left.O \cap W_{y}^{s} \cap \mathcal{F}^{-k} \mathcal{S}_{1}^{+}(\epsilon)\right)$ com $k>0$.

A Condição L4 será deduzida na Proposição 13 de uma propriedade chamada de propriedade de não contração, que nos diz: existe $\beta^{\prime}>0$ tal que se $z \in E \backslash \mathcal{R}_{m}^{+}$e $\mathcal{F}^{m} z \in E \cup-E \operatorname{com} m>0$, então

$$
\left\|D_{z} \mathcal{F}^{m} v\right\| \geq \beta^{\prime}\|v\| \quad \text { para } v \in C(z) .
$$

onde $\left\{U_{z}, C(z)\right\}_{z \in E}$ é o campo de cone definido no Capítulo 3. Essa propriedade será verificada decompondo as órbitas do bilhar em blocos especiais, e estudamos (4.4) separadamente para cada bloco. Essa análise será feita usando certas semi-normas definidas em termos de campos transversais de Jacobi ao longo das órbitas do bilhar.

Aqui é necessário trabalhar em todo $M$, uma vez que ao tomarmos $v$ "entrando"em $\mathcal{M}^{0}$, a Condição L4 nos diz que a saída da faixa deverá ser do tipo "boa", isto é, não poderá haver contração de vetores instáveis (resp. estáveis). Dessa forma, o campo de cone em $\mathcal{M}^{0}$ é definido da seguinte forma: dados $x \in \mathcal{M}^{+} \cup \mathcal{M}^{-}$tal que $\mathfrak{F} x \in \mathcal{M}^{0}$ e $v \in T_{x} \mathcal{M}$, definimos $C_{\mathfrak{F} x}:=D_{x} \mathcal{F} C_{x}$.

\subsubsection{Campos de Jacobi e semi-normas.}

Para mais detalhes veja [CM06], Seção 3.6. Seja $v \in T_{x} M \operatorname{com} x \in M$, e considere a variação $\left\{l^{+}(\sigma)\right\}$ introduzida na Seção 2.5. O campo de Jacobi $J(t)$ associado à essa variação é dado por

$$
J(t)=\left.\frac{\partial l_{\sigma}^{+}(t)}{\partial \sigma}\right|_{\sigma=0}=q^{\prime}(0)+t u^{\prime}(0)
$$

onde ' denota a derivada com respeito à $\sigma$. Então para cada $t \in \mathbb{R}$, tem-se

$$
J^{\prime}(t):=\frac{d J}{d t}=u^{\prime}(0) \quad \text { e } \quad J(t)=J(0)+t J^{\prime}(0) .
$$

Assim, vemos que existe uma correspondência biunívoca entre um vetor $v \in T_{x} M$ e um vetor $\left(J, J^{\prime}\right) \in \mathbb{R}^{2}$ tal que $\langle J(0), u(0)\rangle=\left\langle J^{\prime}(0), u(0)\right\rangle=0$. O par $\left(J(0), J^{\prime}(0)\right)$ define um campo de Jacobi ao longo do segmento $\left[q(0), q_{1}(0)\right]$. Tal campo de Jacobi é chamado de transversal. Escrevemos $J$ e $J^{\prime}$ para $J(0)$ e $J^{\prime}(0)$ respectivamente.

Observamos que o par $\left(J, J^{\prime}\right)$ forma um sistema de coordenadas para o espaço tangente $T_{x} M$. De fato, a mudança de coordenadas $(d s, d \alpha) \mapsto\left(J, J^{\prime}\right)$ é dada por

$$
J=\cos \alpha d s \quad \text { e } \quad J^{\prime}=-\mathcal{K} d s+d \alpha .
$$

Para salientar a dependência de $J$ e $J^{\prime}$ no vetor $v$, escrevemos $J(v)$ e $J^{\prime}(v)$. Quando não há colisão com $\partial \Omega$ durante um intervalo de comprimento $t \in \mathbb{R}$, a evolução de $\left(J, J^{\prime}\right)$ é dada pela transformação 
linear $F(t)$. Em uma colisão $x \in M \backslash \partial M$, o par $\left(J, J^{\prime}\right)$ é transformado de acordo com a transformação linear $R(x)$. As transformações $F(t)$ e $R(x)$ são dadas por:

$$
F(t)=\left(\begin{array}{ll}
1 & \tau \\
0 & 1
\end{array}\right) \quad \text { e } \quad R(y)=\left(\begin{array}{cc}
-1 & 0 \\
\frac{2 \mathcal{K}(x)}{\cos \alpha(x)} & -1
\end{array}\right) .
$$

Portanto, a matriz de $D_{x} \mathcal{F}$ em coordenadas $J$ e $J^{\prime}$ é dada por

$$
D_{x} \mathcal{F}=R(\mathcal{F} x) F(\tau(x))=\left(\begin{array}{cc}
-1 & -\tau \\
\frac{2 \mathcal{K}_{1}}{\cos \alpha_{1}} & -1+\frac{2 \tau \mathcal{K}_{1}}{\cos \alpha_{1}}
\end{array}\right) .
$$

Observamos que todas as matrizes em (4.5) e (4.6) tem determinante igual a \pm 1 . Isso significa que em coordenadas $J$ e $J^{\prime}$, a transformação $D_{x} \mathcal{F}$ preserva a forma simplética standard $J \wedge J^{\prime}$. Finalmente, observamos que o tempo de focalização para frente de um vetor tangente $v \in T_{x} M$ com $x \in M$ em termos de $J(v)$ e $J^{\prime}(v)$ é dado por

$$
f^{+}(v)=\left\{\begin{array}{cc}
\frac{-J(v)}{J^{\prime}(v)}, & J^{\prime}(v) \neq 0 \\
\infty, & J^{\prime}(v)=0 .
\end{array}\right.
$$

Definição 15. Para cada $x \in M \backslash \partial M$ e cada $v \in T_{x} M$, defina

$$
\|v\|_{J}=\sqrt{J^{2}(v)+J^{\prime 2}(v)} \quad \text { e }|v|_{J^{\prime}}=\left|J^{\prime}(v)\right| .
$$

Agora, iremos provar diversas relações envolvendo as semi-normas $\|\cdot\|$ e $\|\cdot\|_{J}$. O objetivo é mostrar que $\|\cdot\|$ e $\|\cdot\|_{J}$ são equivalentes em um certo subconjunto do fibrado tangente $T M$. O campo de cone $\left\{U_{x}, C_{i}(x)\right\}_{x \in E}, i \in\{0,1,2\}$, usado nas proposições abaixo são aqueles introduzidos no Capítulo 4.

Os próximos Lemas e Proposições seguem na mesma ordem que em [MM14].

Lema 6. Existe uma constante $a_{1}>1$ dependendo somente em $\Omega$ e a família de campo de cones $\left\{U_{x}, C_{i}(x)\right\}_{x \in E^{+}}, i \in\{1,2\}$, tal que se $x \in E^{+}, z \in U_{x}$ e $0 \leq k \leq n(z)$, então

$$
\left|D_{z} \mathcal{F}^{k} v\right|_{J^{\prime}} \leq\left\|D_{z} \mathcal{F}^{k} v\right\|_{J} \leq a_{1}\left|D_{z} \mathcal{F}^{k} v\right|_{J^{\prime}} \quad \text { para } v \in C_{i}(z), \quad i \in\{1,2\} .
$$

Demonstração. Sejam $x, z, k, v$ como na hipótese do lema. Denotamos por $z_{k}=\mathcal{F}^{k} z$ e $v_{k}=D_{z} \mathcal{F}^{k} v$, para $0 \leq k \leq n(z)$. É claro que $\left|v_{k}\right|_{J^{\prime}} \leq\left\|v_{k}\right\|_{J}$. Agora, se $v_{k} \in C_{2}\left(z_{k}\right)$ então

$$
f^{+}\left(z_{k}, v_{k}\right) \leq \frac{\cos \alpha_{k}}{\mathcal{K}_{f}}
$$

em que $\cos \alpha_{k} \gg 0$. Assim, por (4.7), temos $\left|J\left(v_{k}\right)\right| \leq \frac{\cos \alpha\left|J^{\prime}\left(v_{k}\right)\right|}{\mathcal{K}_{f}}$. Agora, observe que

$$
\left\|v_{k}\right\|_{J}^{2}=J^{2}\left(v_{k}\right)+J^{\prime 2}\left(v_{k}\right) \leq\left(1+\frac{\cos ^{2} \alpha_{k}}{\mathcal{K}_{f}^{2}}\right) J^{\prime}\left(v_{k}\right)^{2} .
$$

Logo, a desigualdade segue com $a_{1}=\sqrt{1+\frac{\cos ^{2} \alpha_{k}}{\mathcal{K}_{f}^{2}}}$.

Se $v \in C_{1}(z)$ então por definição $f^{+}(z, v) \geq c$, em que $c \ll 0$, daí $f^{+}(z, v)^{2} \leq c^{2}$, e o argumento segue como no caso anterior tomando $a_{1}=\sqrt{1+c^{2}}$.

Assim, concluimos que se $v \in C_{1}(z) \cup C_{2}(z)$ então existe $\bar{c} \in \mathbb{R}$, com $|\bar{c}| \gg 0$, tal que a desigualdade requerida é válida para $a_{1}=\sqrt{1+\bar{c}^{2}}$. 
Lema 7. Existe $a_{2}>1$ tal que se $x \in M \backslash \partial M$, então

$$
\|v\|_{J} \leq a_{2}\|v\| \quad \text { para } v \in T_{x} M .
$$

Demonstração. Primeiro observamos que se $x \in \mathcal{M}^{0}$ então $\mathcal{K}(x)=0$ e a desigualdade segue para qualquer $a_{2}>1$. Suponhamos agora que $x \in \mathcal{M}$. Se $v \in C_{1}(x)$, pela definição do campo de cone $C_{1}(x)$ e pela Fórmula $(2.2)$ temos $d \alpha / d s>0$, assim

$$
\begin{aligned}
\|v\|_{J}^{2}=J^{2}(v)+J^{\prime 2}(v) & =d s^{2}\left(\cos ^{2} \alpha+\mathcal{K}_{f}^{2}\right)+d \alpha^{2}-2 \mathcal{K}_{f} d s d \alpha \\
& \leq\left(\cos ^{2} \alpha+\mathcal{K}_{f}^{2}+1\right)\|v\|^{2}
\end{aligned}
$$

Assim, tome $a_{2}=\sqrt{1+\cos ^{2} \alpha+\mathcal{K}_{f}^{2}}$.

Agora, observamos que se $v \in C_{0}(x)$ então $d \alpha / d s>0$ e se $v \in C_{2}(x)$ então $d \alpha / d s \leq 0$. Logo, $-2 \mathcal{K} d s d \alpha \geq 0$ em que $\mathcal{K}=\mathcal{K}_{d}<0$ para $v \in C_{0}(x)$ e $\mathcal{K}=\mathcal{K}_{f}>0$ para $v \in C_{2}(x)$. Assim, dado $v \in C_{0}(x) \cup C_{2}(x)$ temos

$$
\begin{aligned}
\frac{\|v\|_{J}^{2}}{d s^{2}+d \alpha^{2}} & =\frac{d s^{2}}{d s^{2}+d \alpha^{2}}\left(\cos ^{2} \alpha+\mathcal{K}^{2}\right)+\frac{d \alpha^{2}}{d s^{2}+d \alpha^{2}}-\frac{2 \mathcal{K} d s d \alpha}{d s^{2}+d \alpha^{2}} \\
& \leq \cos ^{2} \alpha+\mathcal{K}^{2}+1-\frac{2 \mathcal{K} d s d \alpha}{d s^{2}+d \alpha^{2}}
\end{aligned}
$$

Daí, tome $a_{2}=\sqrt{\cos ^{2} \alpha+\mathcal{K}^{2}+1-\frac{2 \mathcal{K} d s d \alpha}{d s^{2}+d \alpha^{2}}}$.

Lema 8. Existe $a_{3}>0$ tal que se $x \in \mathcal{M}^{-}$e $z \in U_{x}$ então

$$
\|v\| \leq a_{3}\|v\|_{J} \quad \text { para } v \in C_{0}(z) .
$$

Demonstração. Dado $v \in C_{0}(z)$ novamente $d \alpha / d s>0$ e, assim,

$$
\begin{aligned}
\|v\|_{J}^{2}=J^{2}(v)+J^{\prime 2}(v) & =d s^{2}\left(\cos ^{2} \alpha+\mathcal{K}_{d}^{2}\right)+d \alpha^{2}-2 \mathcal{K}_{d} d s d \alpha \\
& \geq \frac{\cos ^{2} \alpha+\mathcal{K}_{d}^{2}}{\cos ^{2} \alpha+\mathcal{K}_{d}^{2}+1}\left(d s^{2}+d \alpha^{2}\right)
\end{aligned}
$$

logo, tome $a_{3}=\sqrt{\frac{\cos ^{2} \alpha+\mathcal{K}_{d}^{2}+1}{\cos ^{2} \alpha+\mathcal{K}_{d}^{2}}}$.

Lema 9. Existe $a_{4}>0$ tal que se $x \in E^{+}, z \in U_{x}$ e $v \in C_{i}(z), i \in\{1,2\}$, então

$$
\left\|D_{z} \mathcal{F}^{k} v\right\| \leq a_{4}\left\|D_{z} \mathcal{F}^{k} v\right\|_{J} \quad \text { para } 0 \leq k \leq n(z) .
$$

Demonstração. Novamente, denotamos por $z_{k}=\mathcal{F}^{k} z$ e $v_{k}=D_{z} \mathcal{F}^{k} v$. Se $v \in C_{2}(z)$ então $v_{k} \in$ $C_{2}\left(z_{k}\right)$ para $0 \leq k \leq n(z)$ e, assim, $d \alpha / d s_{k} \leq 0$. Logo,

$$
\begin{aligned}
\left\|v_{k}\right\|_{J}^{2}=J^{2}\left(v_{k}\right)+J^{\prime 2}\left(v_{k}\right) & =d s_{k}^{2}\left(\cos ^{2} \alpha+\mathcal{K}_{f}^{2}\right)+d \alpha^{2}-2 \mathcal{K}_{f} d s_{k} d \alpha \\
& \geq \frac{\cos ^{2} \alpha+\mathcal{K}_{f}^{2}}{\cos ^{2} \alpha+\mathcal{K}_{f}^{2}+1}\left(d s_{k}^{2}+d \alpha^{2}\right)
\end{aligned}
$$


Portanto, tome $a_{4}=\sqrt{\frac{\cos ^{2} \alpha+\mathcal{K}_{f}^{2}+1}{\cos ^{2} \alpha+\mathcal{K}_{f}^{2}}}$.

Se $v \in C_{1}(z)$ então notamos que $d \alpha / d s>0$, assim

$$
\begin{aligned}
\frac{\left\|v_{k}\right\|_{J}^{2}}{d s_{k}^{2}} & =\left(\cos ^{2} \alpha+\mathcal{K}_{f}^{2}\right)+\left(\frac{d \alpha}{d s_{k}}\right)^{2}-2 \mathcal{K}_{f} \frac{d \alpha}{d s_{k}} \\
& \geq \cos ^{2} \alpha \gg 0
\end{aligned}
$$

pois $\mathcal{F}^{-1} z \in \mathcal{M}^{-}$. Logo, basta tomar $a_{4} \geq \frac{d s_{k}^{2}+d \alpha^{2}}{d s_{k}^{2} \cos ^{2} \alpha}$ e temos a desigualdade pedida.

Lema 10. Existe $\epsilon_{0}>0$ e $\alpha_{0} \in(0, \pi / 2)$ tal que $\alpha\left(\mathcal{M}^{0} \cap \mathcal{S}_{1}^{-}\left(\epsilon_{0}\right)\right) \in\left(\alpha_{0}-\pi / 2, \alpha_{0}\right)$.

Demonstração. O lema é consequência do seguinte fato: seja $\Gamma_{i}$ uma componente flat de $\partial \Omega$; então dado qualquer vértice $p$ de $\Gamma_{i}$, nenhum raio emergindo de elementos de $\mathcal{M}_{i} \cap \mathcal{S}_{1}^{+}$contém $p$.

Lema 11. Seja $\epsilon_{0}$ a constante do Lema 10. Então existe $a_{5}>0$ tal que se $x \in \mathcal{M}^{0} \cap \mathcal{S}_{1}^{-}\left(\epsilon_{0}\right)$, então

$$
\|v\| \leq a_{5}\|v\|_{J} \quad \text { para } v \in T_{x} M .
$$

Demonstração. Como $\mathcal{K}(x)=0$, segue que $\|v\|_{J}^{2}=\cos ^{2} \alpha d s^{2}+d \alpha^{2} \geq \cos ^{2} \alpha\|v\|^{2}$.

Corolário 4. Existem duas constantes $0<A_{1}<A_{2}$ tais que se $x \in E, z \in U_{x}$ e $v \in C_{i}(z)$, $i \in\{0,1,2\}$, então

$$
A_{1}\|v\|_{J} \leq\|v\| \leq A_{2}\|v\|_{J} .
$$

Demonstração. Segue dos Lemas 7-11.

Observação 8. O Corolário anterior, usando o Lema 6, ainda permanece válido se substituirmos $\|\cdot\| \operatorname{por}|\cdot|_{J^{\prime}}$, mostrando assim que as semi-normas $\|\cdot\|,\|\cdot\|_{J},|\cdot|_{J^{\prime}}$ são equivalentes no sentido especificado no corolário.

\subsubsection{Blocos de decomposição.}

Seguindo [MM14], iremos decompor uma sequência de colisões $\left\{z, \ldots, \mathcal{F}^{m} z\right\}$ em um número finito de subsequências chamada blocos.

Definição 16. Seja $z \in M \backslash \mathcal{R}_{m}^{+} \operatorname{com} m>0$.

- Chamamos de bloco do tipo 1 se: $z \in \mathcal{M}^{-},\left\{\mathfrak{F} z, \ldots, \mathfrak{F}^{m-1} z\right\} \subset \mathcal{M}^{0} \cup \mathcal{M}^{-}$e $\mathcal{F}^{m} z \in \mathcal{M}^{-}$.

- Chamamos de bloco do tipo 2 se: $z \in \mathcal{M}^{-},\left\{\mathfrak{F} z, \ldots, \mathfrak{F}^{m-1} z\right\} \subset \mathcal{M}^{0}$ e $\mathcal{F}^{m} z \in E^{+}$.

- Chamamos de bloco do tipo 3 se: $z \in E^{+},\left\{\mathfrak{F}^{n(z)+1} z, \ldots, \mathfrak{F}^{m-1} z\right\} \subset \mathcal{M}^{0}$ e $\mathcal{F}^{m} z \in \mathcal{M}^{-}$.

- Chamamos de bloco do tipo 4 se: $z$ e $\mathcal{F}^{m} z$ pertencem à $E^{+}$.

Definição 17. Um bloco é chamado minimal se não contém qualquer outro bloco do mesmo tipo. Um bloco incluido em uma sequência de colisões consecutivas $\varphi$ é chamada maximal em $\varphi$ se não contém qualquer outro bloco do mesmo tipo em $\varphi$.

Observamos que blocos do tipo 2 e 3 são sempre minimal e maximal. Também observamos que cada bloco do tipo 1 e 4 é uma união de um número finito de blocos minimais do tipo 1 e 4 , respectivamente. A próxima Proposição nos diz que podemos decompor nosso bilhar em um número finito de blocos. Sua demonstração pode ser encontrada em [MM14], Proposição 7.12.

Proposição 9. Seja $\varphi=\left\{z, \ldots, \mathcal{F}^{m} z\right\}$ uma sequência de colisões tal que $z$ e $\mathcal{F}^{m} z$ pertençam à $E$. Então $\varphi=\varphi_{1} \cup \cdots \cup \varphi_{n}$ com $n \leq 5$ e $\varphi_{1}, \ldots, \varphi_{n}$ sendo blocos maximais do tipo 1 e 2. Além disso, essa decomposição é única. 


\subsubsection{Provando a propriedade de não contração.}

É suficiente mostrarmos que (4.4) segue ao longo dos blocos do tipo 1-4 com $\beta^{\prime}$ dependendo somente do tipo do bloco.

Começaremos com alguns resultados preliminares.

Lema 12. Seja $0 \leq m_{1}<m_{2}$, e suponha que $z \in E \backslash \mathcal{R}_{m_{2}}^{+}$e $0 \neq v \in C(z)$. Então

$$
\frac{\left|D_{z} \mathcal{F}^{m_{2}} v\right|_{J^{\prime}}}{\left|D_{z} \mathcal{F}^{m_{1}} v\right|_{J^{\prime}}}=\prod_{k=m_{1}+1}^{m_{2}}\left|\frac{f^{-}\left(z_{k}, v_{k}\right)}{f^{+}\left(z_{k}, v_{k}\right)}\right|,
$$

onde $z_{k}=\mathcal{F}^{k} z$ e $v_{k}=D_{z} \mathcal{F}^{k} v$.

Demonstração. Primeiramente façamos algumas observações: seja $0 \neq v \in C(z)$, por (4.7) se $J^{\prime}\left(v_{k}\right) \neq 0$ para $0 \leq k \leq m_{2}$ temos $f^{+}\left(z_{k}, v_{k}\right)=-J\left(v_{k}\right) / J^{\prime}\left(v_{k}\right)$ e pelo Lema 1 tem-se

$$
f^{-}\left(v_{k}\right)=\frac{J\left(v_{k}\right) \cos \alpha_{k}}{-J^{\prime}\left(v_{k}\right) \cos \alpha_{k}-2 \mathcal{K}\left(z_{k}\right) J\left(v_{k}\right)} .
$$

Aplicando a matriz da derivada dada em (4.6) vemos que,

$$
\begin{gathered}
J\left(v_{k}\right)=-J\left(v_{k-1}\right)-\tau\left(z_{k-1}\right) J^{\prime}\left(v_{k-1}\right), \quad \text { e } \\
J^{\prime}\left(v_{k}\right)=\frac{2 \mathcal{K}\left(z_{k}\right)}{\cos \alpha_{k}} \cdot J\left(v_{k-1}\right)+2 \tau\left(z_{k-1}\right) J^{\prime}\left(v_{k-1}\right) \cdot \frac{\mathcal{K}\left(z_{k}\right)}{\cos \alpha_{k}}-J^{\prime}\left(v_{k-1}\right),
\end{gathered}
$$

substituindo as duas equações acima em (4.8) obtemos $f^{-}\left(v_{k}\right)=J\left(v_{k}\right) / J^{\prime}\left(v_{k-1}\right)$.

Assim,

$$
\begin{aligned}
\frac{\left|v_{m_{2}}\right|_{J^{\prime}}}{\left|v_{m_{1}}\right|_{J^{\prime}}} & =\prod_{k=m_{1}+1}^{m_{2}}\left|\frac{J^{\prime}\left(v_{k}\right)}{J\left(v_{k}\right)}\right| \cdot\left|\frac{J\left(v_{k}\right)}{J^{\prime}\left(v_{k-1}\right)}\right| \\
& =\prod_{k=m_{1}+1}^{m_{2}}\left|\frac{f^{-}\left(z_{k}, v_{k}\right)}{f^{+}\left(z_{k}, v_{k}\right)}\right|
\end{aligned}
$$

se $f^{+}\left(z_{k}, v_{k}\right) \neq 0$, isto é, se $J\left(v_{k}\right) \neq 0$ para $1 \leq k \leq m_{2}$.

Sendo assim, é suficiente provar que $J^{\prime}\left(v_{k}\right) \neq 0$ para $0 \leq k \leq m_{2}$ e $J\left(v_{k}\right) \neq 0$ para $1 \leq k \leq m_{2}$.

De fato, suponhamos inicialmente que $z_{k}=\mathcal{F}^{k} z \in \mathcal{M}^{-}$para todo $0 \leq k \leq m_{2}$. Logo, seja $v \in C_{0}(z)$. Se $J^{\prime}\left(v_{k}\right)=0$ para algum $0 \leq k \leq m_{2}$, segue que $-\mathcal{K}_{d} d s_{k}+d \alpha_{k}=0$ isso implica que $d \alpha_{k} / d s_{k}=\mathcal{K}_{d}<0$, absurdo pois como $v_{k} \in C_{0}\left(z_{k}\right)$ e usando (2.2) temos $d \alpha_{k} / d s_{k} \geq 0$. Se $J\left(v_{k}\right)=0$ para algum $1 \leq k \leq m_{2} \operatorname{logo}$ por (4.9) temos $-J\left(v_{k-1}\right)-\tau\left(z_{k-1}\right) J^{\prime}\left(v_{k-1}\right)=0$ que implica

$$
f^{+}\left(z_{k-1}, v_{k-1}\right)=-\frac{J\left(v_{k-1}\right)}{J^{\prime}\left(v_{k-1}\right)}=\tau\left(z_{k-1}\right) \gg 0,
$$

o que não ocorre, pois $v_{k-1} \in C_{0}\left(z_{k-1}\right)$, isto é, $f^{+}\left(z_{k-1}, v_{k-1}\right) \leq 0$.

Suponhamos agora que $z_{k}=\mathcal{F}^{k} z \in \mathcal{M}^{+}$para $0 \leq k \leq m_{2}$ (caso exista $1 \leq k \leq m_{2}$ tal que $z_{k} \in \mathcal{M}^{-}$, aplicamos o argumento anterior). Sendo assim $v \in C_{1}(z) \cup C_{2}(z)$. Inicialmente, suponhamos que $v \in C_{1}(z)$. Se $J^{\prime}(v)=0$ então, por $(4.7), f^{+}(z, v)=\infty$, o que não ocorre (veja argumento (2.1) da prova da Proposição 7). Se $J^{\prime}\left(v_{k}\right)=0$ para algum $1 \leq k \leq m_{2}$, então também por (4.7), $f^{+}\left(z_{k}, v_{k}\right)=\infty$, um absurdo pois $v_{k} \in C_{2}\left(z_{k}\right)$ (com ou sem colisões com $\Gamma^{0}$ entre $z_{k-1}$ e $\left.z_{k}\right)$. Se $J\left(v_{k}\right)=0$, para algum $1 \leq k \leq m_{2}$, então $-J\left(v_{k-1}\right)-\tau\left(z_{k-1}\right) J^{\prime}\left(v_{k-1}\right)=0$ que implica $f^{+}\left(z_{k-1}, v_{k-1}\right)=\tau\left(z_{k-1}\right) \gg 0$. Agora, para $k=1$ temos um absurdo pois $v \in C_{1}(z)$, e para $2 \leq k \leq$ 
$m_{2}$ considerando que não há colisão com $\Gamma^{0}$ entre $z_{k-1}$ e $z_{k}$, temos $f^{+}\left(z_{k-1}, v_{k-1}\right)=(2 \cos \alpha) / \mathcal{K}_{f}$, absurdo pois $v_{k-1} \in C_{2}\left(z_{k-1}\right)$. Se houver pelo menos uma colisão com $\Gamma^{0}$ entre $z_{k-1}$ e $z_{k}$, teríamos $f^{+}\left(z_{k-1}, v_{k-1}\right)=\tau\left(z_{k-1}\right)>2 / \mathcal{K}_{f}$ o que também não cumpre $v_{k-1} \in C_{2}\left(z_{k-1}\right)$. Suponha agora que $v \in C_{2}(z)$. Nesse caso, se $J^{\prime}\left(v_{k}\right)=0$ para $0 \leq k \leq m_{2}$ então por (4.7) $f^{+}\left(z_{k}, v_{k}\right)=\infty$ o que não ocorre, pois $v_{k} \in C_{2}\left(z_{k}\right)$ para todo $0 \leq k \leq m_{2}$. Se $J\left(v_{k}\right)=0$ para algum $1 \leq k \leq m_{2}$, então $f^{+}\left(z_{k-1}, v_{k-1}\right)=\tau\left(z_{k-1}\right)=(2 \cos \alpha) / \mathcal{K}_{f}$ (se não houver colisão com $\Gamma^{0}$ entre $z_{k-1}$ e $z_{k}$ ), e $f^{+}\left(z_{k-1}, v_{k-1}\right)=\tau\left(z_{k-1}\right)>2 / \mathcal{K}_{f}$ (se há pelo menos uma colisão). Em qualquer um dos casos implica que $v_{k} \notin C_{2}\left(z_{k}\right)$, o que é um absurdo.

Lema 13. Considere uma sequência de colisões $\left\{z, \ldots, \mathcal{F}^{m} z\right\}$ com $m>0$ tal que $z, \mathcal{F}^{m} z \in E$, e $\mathfrak{F}^{k} z \in \mathcal{M}^{0}$ para $n(z)<k<m$. Então, para cada $0 \neq v \in C(z)$ e para cada $n(z) \leq k<m$ tem-se

$$
\left|D_{z} \mathcal{F}^{m} v\right|_{J^{\prime}}>\left|D_{z} \mathcal{F}^{k} v\right|_{J^{\prime}}
$$

Demonstração. Seja $0 \neq v \in C(z)$ e defina $z_{k}=\mathcal{F}^{k} z$ e $v_{k}=D_{z} \mathcal{F}^{k} v$ para $0 \leq k \leq m$. Pelo Lema 1 segue que

$$
\frac{f^{-}\left(z_{k}, v_{k}\right)}{f^{+}\left(z_{k}, v_{k}\right)}=\frac{\cos \alpha_{k}}{\cos \alpha_{k}-2 \mathcal{K} f^{+}\left(z_{k}, v_{k}\right)}
$$

Pelo Lema 12 e usando o fato que $\mathcal{K}=0$ para $n(z)+1 \leq k \leq m-1$, temos:

$$
\frac{\left|v_{m}\right| J_{J^{\prime}}}{\left|v_{k}\right|_{J^{\prime}}}=\prod_{j=k+1}^{m}\left|\frac{f^{-}\left(z_{j}, v_{j}\right)}{f^{+}\left(z_{j}, v_{j}\right)}\right|=\left|\frac{f^{-}\left(z_{m}, v_{m}\right)}{f^{+}\left(z_{m}, v_{m}\right)}\right|=\left|\frac{\cos \alpha_{m}}{\cos \alpha_{m}-2 \mathcal{K} f^{+}\left(z_{m}, v_{m}\right)}\right| \text {. }
$$

Agora, se $z_{m} \in \mathcal{M}^{-}$, então $\mathcal{K}=\mathcal{K}_{d}<0$ e $v_{m} \in C_{0}\left(z_{m}\right)$. Por invariância estrita, temos $F_{m}^{+} \in$ $D_{-4}^{\circ}\left(z_{m}\right) \subset D_{-2}^{\circ}\left(z_{m}\right)$ (veja III.2.2, [BL08]) daí $\cos \alpha_{m} / 2 \mathcal{K}_{d}<f^{+}\left(z_{m}, v_{m}\right)<0$. Isso significa que

$$
0<\cos \alpha_{m}-2 \mathcal{K}_{d} f^{+}\left(z_{m}, v_{m}\right)<\cos \alpha_{m}
$$

$\log \left|f^{-}\left(z_{m}, v_{m}\right) / f^{+}\left(z_{m}, v_{m}\right)\right|>1$.

Se $z_{m} \in \mathcal{M}^{+}$, então $\mathcal{K}=\mathcal{K}_{f}>0$ e $v_{m} \in C_{2}\left(z_{m}\right)$. Novamente por invariância estrita (IV.2.2, [BL08]), vemos que $F_{1}^{+} \in D_{2}^{\circ}\left(z_{m}\right) \backslash D_{4}\left(z_{m}\right)$ e assim, $\left(\cos \alpha_{m}\right) / 2 \mathcal{K}_{f}<f^{+}\left(z_{m}, v_{m}\right)<\cos \alpha_{m} / \mathcal{K}_{f}$. Daí,

$$
-\cos \alpha_{m}<\cos \alpha_{m}-2 \mathcal{K}_{f} f^{+}\left(z_{m}, v_{m}\right)<0
$$

isso significa que

$$
\frac{\cos \alpha_{m}}{\cos \alpha_{m}-2 \mathcal{K}_{f} f^{+}\left(z_{m}, v_{m}\right)}<-1
$$

ou seja, $\left|\frac{f^{-}\left(z_{m}, v_{m}\right)}{f^{+}\left(z_{m}, v_{m}\right)}\right|>1$.

Lema 14. Considere uma sequência de colisões $\left\{z, \ldots, \mathcal{F}^{m} z\right\}$ com $m>0$ tal que $z \in \mathcal{M}^{-}, \mathcal{F}^{m} z \in E$ $e \mathfrak{F}^{k} z \in \mathcal{M}^{0}$ para $n(z)<k<m$. Então, para cada $0 \neq v \in C_{0}(z)$ e cada $0 \leq k<m$, tem-se

$$
\left|J\left(D_{z} \mathcal{F}^{m} v\right)\right|>\left|J\left(D_{z} \mathcal{F}^{k} v\right)\right| .
$$

Demonstração. Dado $0 \neq v \in C_{0}(z)$, temos $\cos \alpha / \mathcal{K}_{d} \leq f^{+}(v)<0$, e assim $d s d \alpha>0 \operatorname{logo}$ $J(v) J^{\prime}(v)>0$ e $J^{\prime}(v) \neq 0$ (caso contrário $f^{+}(v)=\infty$ ). Agora, como $\left\{\mathfrak{F} z, \ldots, \mathfrak{F}^{m-1} z\right\} \subset \mathcal{M}^{0}$ e usando (4.10), obtemos $\left|J^{\prime}\left(v_{k}\right)\right|=\left|J^{\prime}(v)\right|$ para $0 \leq k<m$ e por (4.9) tem-se,

$$
J\left(v_{k}\right)=(-1)^{k}\left[J(v)+J^{\prime}(v) \sum_{i=0}^{k-1} \tau\left(z_{i}\right)\right] .
$$


Usando o fato acima que $J(v) J^{\prime}(v)>0$, obtemos

$$
\left|J\left(v_{k}\right)\right|=|J(v)|+\left|J^{\prime}(v)\right| \sum_{i=0}^{k-1} \tau\left(z_{i}\right) \quad \text { para } \quad 0 \leq k<m .
$$

Utilizando a Equação (4.9) para $k=m$, obtemos

$$
J\left(v_{m}\right)=-J\left(v_{m-1}\right)-\tau\left(z_{m-1}\right) J^{\prime}\left(v_{m-1}\right),
$$

daí

$$
\left|J\left(v_{m}\right)\right|=\left|J\left(v_{m-1}\right)+\tau\left(z_{m-1}\right) J^{\prime}\left(v_{m-1}\right)\right|
$$

Pela Fórmula (4.10), sabemos que $J^{\prime}\left(v_{m-1}\right)=(-1)^{m-1} J^{\prime}(v)$, e usando (4.11) com $k=m-1$ em (4.13), obtemos

$$
\begin{aligned}
\left|J\left(v_{m}\right)\right| & =\left|(-1)^{m-1}\left[J(v)+J^{\prime}(v) \sum_{i=0}^{m-2} \tau\left(z_{i}\right)\right]+(-1)^{m-1} \tau\left(z_{m-1}\right) J^{\prime}(v)\right| \\
& =\left|(-1)^{m-1}\left[J(v)+J^{\prime}(v) \sum_{i=0}^{m-1} \tau\left(z_{i}\right)\right]\right| .
\end{aligned}
$$

Novamente usando o fato que $J(v) J^{\prime}(v)>0$, segue que

$$
\left|J\left(v_{m}\right)\right|=|J(v)|+\left|J^{\prime}(v)\right| \sum_{i=0}^{m-1} \tau\left(z_{i}\right) .
$$

Como $m>k$ segue o resultado.

Lema 15. Existe uma constante $\delta_{1}>0$ tal que para cada sequência de colisões consecutivas $\left\{z, \ldots, \mathcal{F}^{m} z\right\}$ com $z \in E^{+}, \mathcal{F}^{m} z \in E$ e $\mathfrak{F}^{k} z \in \mathcal{M}^{0}$ para $n(z)<k<m$, tem-se

$$
\left\|D_{z} \mathcal{F}^{m} v\right\| \geq \delta_{1}\left\|D_{z} \mathcal{F}^{n(z)} v\right\| \quad \text { para } \quad v \in C_{1}(z) \cup C_{2}(z) .
$$

Demonstração. Se substituirmos $\|\cdot\| \operatorname{com}|\cdot|_{J^{\prime}}$, então o resultado segue com $\delta_{1}=1$ do Lema 13. Caso contrário, vemos que

$$
\frac{\left\|D_{z} \mathcal{F}^{m} v\right\|}{\left\|D_{z} \mathcal{F}^{n(z)} v\right\|} \geq \frac{\left\|D_{z} \mathcal{F}^{m} v\right\|_{J}}{a_{2}\left\|D_{z} \mathcal{F}^{n(z)} v\right\|} \geq \frac{\left\|D_{z} \mathcal{F}^{m} v\right\|_{J}}{a_{2} a_{4}\left\|D_{z} \mathcal{F}^{n(z)} v\right\|_{J}}
$$

onde a primeira desigualdade de (4.14) é obtida pelo Lema 7 com $a_{2}>1$ e a segunda desigualdade pelo Lema 9 com $a_{4}>0$. Agora, pelo Lema 6 , existe $a_{1}>1$ tal que $a_{2} a_{4}\left\|D_{z} \mathcal{F}^{n(z)} v\right\|_{J} \leq$ $a_{1} a_{2} a_{4}\left|D_{z} \mathcal{F}^{n(z)} v\right|_{J^{\prime}}$. Isso implica que,

$$
\frac{\left\|D_{z} \mathcal{F}^{m} v\right\|_{J}}{a_{2} a_{4}\left\|D_{z} \mathcal{F}^{n(z)} v\right\|_{J}} \geq \frac{\left\|D_{z} \mathcal{F}^{m} v\right\|_{J}}{a_{1} a_{2} a_{4}\left|D_{z} \mathcal{F}^{n(z)} v\right|_{J^{\prime}}} \geq \frac{\left|D_{z} \mathcal{F}^{m} v\right|_{J^{\prime}}}{a_{1} a_{2} a_{4}\left|D_{z} \mathcal{F}^{n(z)} v\right|_{J^{\prime}}}>\frac{1}{a_{1} a_{2} a_{4}}
$$

onde a segunda desigualdade de (4.15) usamos o fato óbvio que $\|\cdot\|_{J} \geq|\cdot|_{J^{\prime}}$ e a última desigualdade é obtida pelo Lema 13. Portanto, de (4.14) e (4.15) basta tomar $\delta_{1}=\frac{1}{a_{1} a_{2} a_{4}}$.

Proposição 10. Existe $\gamma_{1}>0$ tal que se $z \in E^{+}$e $0 \leq m_{1}<m_{2} \leq n(z)$, então

$$
\left\|D_{z} \mathcal{F}^{m_{2}} v\right\| \geq \gamma_{1}\left\|D_{z} \mathcal{F}^{m_{1}} v\right\| \quad \text { para } v \in C_{1}(z) \cup C_{2}(z) \text {. }
$$


Demonstração. Observamos que

$$
\left|v_{k}\right|_{J^{\prime}} \leq\left\|v_{k}\right\|_{J} \leq a_{2}\left\|v_{k}\right\| \leq a_{2} a_{4}\left\|v_{k}\right\|_{J} \leq a_{2} a_{4}\left|v_{k}\right|_{J^{\prime}}
$$

em que a primeira desigualdade é obtida pelo Lema 6 , a segunda pelo Lema 7 com $a_{2}>1$, a terceira pelo Lema 9 com $a_{4}>0$ e a última desigualdade é óbvia. Logo,

$$
\frac{1}{a_{2}}\left|v_{k}\right|_{J^{\prime}} \leq\left\|v_{k}\right\| \leq a_{4}\left|v_{k}\right|_{J^{\prime}}
$$

e assim, é suficiente provar a proposição com a norma $\|\cdot\|$ substituída pela semi-norma $|\cdot|_{J^{\prime}}$ e, portanto, a prova fará uso do Lema 12. Seja $v \in C_{1}(z) \cup C_{2}(z)$ e defina $z_{k}=\mathcal{F}^{k} z$ e $v_{k}=D_{z} \mathcal{F}^{k} v$ para $0 \leq k \leq n(z)$. Basicamente devemos estudar os quocientes $f^{-}\left(z_{k}, v_{k}\right) / f^{+}\left(z_{k}, v_{k}\right)$ para $0 \leq k \leq n(z)$.

Pelo Lema 1, tem-se

$$
\frac{f^{-}\left(z_{k}, v_{k}\right)}{f^{+}\left(z_{k}, v_{k}\right)}=\frac{\cos \alpha}{\cos \alpha-2 \mathcal{K}_{f} f^{+}\left(z_{k}, v_{k}\right)} \text {. }
$$

Para $1 \leq k \leq n(z)$ temos $v_{k} \in C_{2}\left(z_{k}\right)$ e assim $\cos \alpha_{k} / 2 \mathcal{K}_{f} \leq f^{+}\left(z_{k}, v_{k}\right) \leq \cos \alpha / \mathcal{K}_{f}$ (com ou sem colisões com $\Gamma^{0}$ entre $z_{k-1}$ e $z_{k}$ ). Usando a demonstração do Lema 13 obtemos

$$
\left|\frac{f^{-}\left(z_{k}, v_{k}\right)}{f^{+}\left(z_{k}, v_{k}\right)}\right|=\frac{\cos \alpha}{\cos \alpha-2 \mathcal{K}_{f} f^{+}\left(z_{k}, v_{k}\right)} \geq 1
$$

Logo, pelo Lema 12 segue que,

$$
\frac{\left|D_{z} \mathcal{F}^{m_{2}} v\right|_{J^{\prime}}}{\left|D_{z} \mathcal{F}^{m_{1}} v\right|_{J^{\prime}}} \geq 1
$$

E assim o Lema segue para $\gamma_{1}=1$. Vemos que o caso $m_{1}=0$ começa no caso $k=1$ no quociente do Lema 12 e assim $v_{k} \in C_{2}\left(v_{k}\right)$ para $1 \leq k \leq n(z)$.

Proposição 11. Existe uma constante $\gamma_{2}>0$ tal que se $z \in E \backslash \mathcal{R}_{m}^{+} \operatorname{com} m>1,\left\{\mathfrak{F}^{n(z)+1} z, \ldots, \mathfrak{F}^{m-1} z\right\} \subset$ $\mathcal{M}^{0}, \mathfrak{F}^{j} z \in \mathcal{S}_{1}^{-}\left(\epsilon_{0}\right)$ para algum $n(z)<j<m$, e $\mathcal{F}^{m} z \in E$, então

$$
\left\|D_{z} \mathcal{F}^{m} v\right\| \geq \gamma_{2}\left\|D_{z} \mathfrak{F}^{j} v\right\|, \quad \text { para } v \in C(z) .
$$

Demonstração. Seja $0 \neq v \in C(z)$ e defina $z_{k}$ e $v_{k}$ para $0 \leq k \leq m$ como na prova do Lema 12. Pelos Lemas 7 e 11 é suficiente provar a desigualdade com $\|\cdot\|$ substituída por $\|\cdot\|_{J}$. Como $z_{k} \in \mathcal{M}^{0}$ para $n(z)<k \leq j$, sabemos por (4.10) que $\left|J^{\prime}\left(v_{j}\right)\right|=\left|J^{\prime}\left(v_{j-1}\right)\right|=\left|J^{\prime}\left(v_{n(z)}\right)\right|$ e utilizando (4.9) obtemos

$$
\begin{aligned}
\left|J\left(v_{j}\right)\right| & =\left|J\left(v_{n(z)}\right)+\tau\left(z_{n(z)}\right) J^{\prime}\left(v_{n(z)}\right)\right| \\
& \leq\left|J\left(v_{n(z)}\right)\right|+\tau\left(z_{n(z)}\right)\left|J^{\prime}\left(v_{n(z)}\right)\right| \\
& =\left|J^{\prime}\left(v_{n(z)}\right)\right|\left|f^{+}\left(z_{n(z)}, v_{n(z)}\right)\right|+\tau\left(z_{n(z)}\right)\left|J^{\prime}\left(v_{n(z)}\right)\right| .
\end{aligned}
$$

em que a última igualdade foi usada (4.7). A prova irá prosseguir da seguinte forma: sabemos pelo fato óbvio que $\left\|v_{m}\right\|_{J} \geq\left|v_{m}\right|_{J^{\prime}}$ e pelo Lema 13 obtemos $\left|v_{m}\right|_{J^{\prime}}>\left|v_{j}\right|_{J^{\prime}}$. Agora, por definição $\left\|v_{j}\right\|_{J}^{2}=J^{2}\left(v_{j}\right)+J^{\prime 2}\left(v_{j}\right)$ e assim basta mostrar que $J\left(v_{j}\right)$ é uma função limitada, que é equivalente a mostrar por (4.16) que $f^{+}\left(z_{n(z)}, v_{n(z)}\right)$ é limitada para todo $v_{n(z)} \in C\left(z_{n(z)}\right)$.

Se $z \in \mathcal{M}^{-}$então por definição $n(z)=0$, assim $\cos \alpha\left(z_{0}\right) / \mathcal{K}_{d} \leq f^{+}\left(z_{0}, v_{0}\right) \leq 0$, logo por (4.16)

$$
\left|J\left(v_{j}\right)\right| \leq\left(\tau\left(z_{0}\right)-\frac{\cos \alpha\left(z_{0}\right)}{\mathcal{K}_{d}}\right)\left|J^{\prime}\left(v_{j}\right)\right| .
$$


Se $z \in \mathcal{M}^{+}$mas $n(z)=0$, temos que $v \in C_{1}(z)$ e assim analisamos $D_{z} \mathcal{F}^{-1} v \in C_{0}\left(\mathcal{F}^{-1} z\right)$, pelo mesmo argumento anterior obtemos

$$
\left|J\left(v_{j}\right)\right| \leq\left(\tau\left(\mathcal{F}^{-1} z\right)-\frac{\cos \alpha\left(\mathcal{F}^{-1} z\right)}{\mathcal{K}_{d}}\right)\left|J^{\prime}\left(v_{j}\right)\right| .
$$

Se $z \in \mathcal{M}^{+} \operatorname{com} 1 \leq n(z)<j$ então $\cos \alpha\left(z_{n(z)}\right) / 2 \mathcal{K}_{f} \leq f^{+}\left(z_{n(z)}, v_{n(z)}\right) \leq \cos \alpha\left(z_{n(z)}\right) / \mathcal{K}_{f}$, assim

$$
\left|J\left(v_{j}\right)\right| \leq\left(\tau\left(z_{n(z)}\right)+\frac{1}{\mathcal{K}_{f}}\right)\left|J^{\prime}\left(v_{j}\right)\right|
$$

Tome $L=\max \left\{\tau\left(z_{0}\right)-\frac{\cos \alpha\left(z_{0}\right)}{\mathcal{K}_{d}}, \tau\left(\mathcal{F}^{-1} z\right)-\frac{\cos \alpha\left(\mathcal{F}^{-1} z\right)}{\mathcal{K}_{d}}, \tau\left(z_{n(z)}\right)+\frac{1}{\mathcal{K}_{f}}\right\}$.

Dessa forma, dado $v_{n(z)} \in C\left(z_{n(z)}\right)$ temos $\left|J\left(v_{j}\right)\right| \leq L\left|J^{\prime}\left(v_{j}\right)\right|$.

Logo,

$$
\left\|v_{j}\right\|_{J}^{2}=J^{2}\left(v_{j}\right)+J^{\prime 2}\left(v_{j}\right) \leq\left(1+L^{2}\right) J^{\prime 2}\left(v_{j}\right)
$$

ou seja, $\left|v_{j}\right|_{J^{\prime}} \geq\left(1+L^{2}\right)^{-1 / 2}\left\|v_{j}\right\|_{J}$. Assim, basta tomar $\gamma_{2}=\left(1+L^{2}\right)^{-1 / 2}$.

Lema 16. Existe $\beta_{1}^{\prime}>0$ tal que cada bloco do tipo 1 satisfaz (4.4) $\operatorname{com} \beta^{\prime}=\beta_{1}^{\prime}$.

Demonstração. Primeiramente, observamos que cada bloco do tipo 1 consiste de um número finito de blocos minimais do tipo 1. Agora, suponhamos que $\left\{z, \ldots, \mathcal{F}^{m} z\right\}$ é um bloco minimal do tipo 1 e seja $0 \neq v \in C_{0}(z)$. Nesse caso, $\mathfrak{F}^{k} z \in \mathcal{M}^{0}$ para $1 \leq k \leq m-1$. Temos que

$$
\left\|D_{z} \mathcal{F}^{m} v\right\|_{J}^{2}=J^{2}\left(D_{z} \mathcal{F}^{m} v\right)+J^{\prime 2}\left(D_{z} \mathcal{F}^{m} v\right)>J^{2}(v)+J^{\prime 2}(v)=\|v\|_{J}^{2},
$$

em que na última desigualdade usamos os Lemas 13 e 14. De fato, a mesma conclusão se estende para blocos gerais do tipo 1 . Para obtermos o resultado na norma $\|\cdot\|$ ao invés de $\|\cdot\|_{J}$, use os Lemas 7 e 8 aplicados à $\left\|D_{z} \mathcal{F}^{m} v\right\|_{J}$ e $\|v\|_{J}$ respectivamente.

Lema 17. Existe $\beta_{2}^{\prime}>0$ tal que cada bloco do tipo 2 satisfaz (4.4) com $\beta^{\prime}=\beta_{2}^{\prime}$.

Demonstração. Suponhamos que $\left\{z, \ldots, \mathcal{F}^{m} z\right\}$ é um bloco do tipo 2 , e seja $0 \neq v \in C_{0}(z)$. Como $\mathfrak{F}^{k} z \in \mathcal{M}^{0}$ para $1 \leq k<m$, tem-se $\left|J^{\prime}\left(D_{z} \mathcal{F}^{m} v\right)\right|>\left|J^{\prime}(v)\right|$ pelo Lema 13 e $\left|J\left(D_{z} \mathcal{F}^{m} v\right)\right|>|J(v)|$ pelo Lema 14. Portanto, $\left\|D_{z} \mathcal{F}^{m} v\right\|_{J}>\|v\|_{J}$. Para a prova completa, aplique os Lemas 7 e 8 à $\left\|D_{z} \mathcal{F}^{m} v\right\|_{J}$ e $\|v\|_{J}$ respectivamente.

Lema 18. Existe $\beta_{3}^{\prime}>0$ tal que cada bloco do tipo 3 satisfaz (4.4) $\operatorname{com} \beta^{\prime}=\beta_{3}^{\prime}$.

Demonstração. Sejam $\varphi=\left\{z, \ldots, \mathcal{F}^{m} z\right\}$ um bloco do tipo 3 e $0 \neq v \in C_{1}(z) \cup C_{2}(z)$. Pela Proposição 10, temos $\left\|D_{z} \mathcal{F}^{n(z)} v\right\| \geq \gamma_{1}\|v\|$. Para a prova completa basta usar Lema 15.

Lema 19. Existe $\beta_{4}^{\prime}>0$ tal que cada bloco do tipo 4 satisfaz (4.4) com $\beta^{\prime}=\beta_{4}^{\prime}$.

Demonstração. Seja $\left\{z, \ldots, \mathcal{F}^{m} z\right\}$ um bloco do tipo 4 . Se $\mathcal{F}^{k} z \in E^{+}$para todo $0 \leq k \leq m$ então o resultado segue pela Proposição 10. Agora, tome bloco minimal onde $z_{k} \in \mathcal{M}^{0} \cup \mathcal{M}^{-}$para todo $1 \leq k \leq m-1$. Se $z_{k} \in \mathcal{M}^{-}$para algum $1 \leq k \leq m-1$ segue que $v_{k} \in C_{0}\left(z_{k}\right)$ e assim pelo Lema 1 sabemos que

$$
\frac{f^{-}\left(z_{k}, v_{k}\right)}{f^{+}\left(z_{k}, v_{k}\right)}=\frac{\cos \alpha\left(z_{k}\right)}{\cos \alpha\left(z_{k}\right)-2 \mathcal{K}_{d} f^{+}\left(z_{k}, v_{k}\right)}
$$

Como $\cos \alpha\left(z_{k}\right) / \mathcal{K}_{d} \leq f^{+}\left(z_{k}, v_{k}\right) \leq 0$ segue que $\left|\cos \alpha\left(z_{k}\right)-2 \mathcal{K}_{d} f^{+}\left(z_{k}, v_{k}\right)\right| \leq \cos \alpha\left(z_{k}\right)$ e assim 
$\left|f^{-}\left(z_{k}, v_{k}\right) / f^{+}\left(z_{k}, v_{k}\right)\right| \geq 1$. Agora, observamos que se $z_{k} \in \mathcal{M}^{0}$ temos $f^{-}\left(z_{k}, v_{k}\right) / f^{+}\left(z_{k}, v_{k}\right)=1$. Em ambos os casos temos pelo Lema 12 e a prova da Proposição 10 que

$$
\frac{\left|D_{z} \mathcal{F}^{m} v\right|_{J^{\prime}}}{|v|_{J^{\prime}}} \geq 1
$$

Se $\mathfrak{F}^{k} z \in \mathcal{M}^{0}$ para $n(z)<k<m$, aplicamos o Lema 13.

Corolário 5. Se $\mathcal{F}^{m} z \in E$, então (4.4) é satisfeita.

Demonstração. Se $\mathcal{F}^{m} z \in E$, então (4.4) segue dos Lemas 16-19.

Proposição 12. A propriedade de não contração é satisfeita.

Demonstração. Seja $\varphi=\left\{z, \ldots, \mathcal{F}^{m} z\right\} \operatorname{com} z \in E$ e $\mathcal{F}^{m} z \in-E$. Como $\mathcal{M}^{-} \subset E$ e $-\mathcal{M}^{-}=\mathcal{M}^{-}$, é suficiente provar a proposição para o caso $\mathcal{F}^{m} z \in-E^{+}$. Se $\mathcal{F}^{m} z \in-E^{+} \cap E^{+}$, então não há nada o que provar. Assim, suponha que $\mathcal{F}^{m} z \notin E^{+}$.

Então temos dois casos: $\varphi \subset \mathcal{M}^{+}$ou $\varphi \not \subset \mathcal{M}^{+}$. Se $\varphi \subset \mathcal{M}^{+}$temos $z \in E^{+}$e $m=n(z)$, então (4.4) é satisfeita pela Proposição 10 tomando $\beta^{\prime}=\gamma_{1}$. Se $\varphi \not \subset \mathcal{M}^{+}$, podemos escrever $\varphi=\varphi_{1} \cup \varphi_{2}$ onde $\varphi_{1}=\left\{z, \ldots, \mathcal{F}^{k} z\right\}$ com $z, \mathcal{F}^{k} z \in E$ e $\varphi_{2}=\left\{\mathcal{F}^{k} z, \ldots, \mathcal{F}^{m} z\right\} \operatorname{com} \mathcal{F}^{k} z \in E^{+}$e $\mathcal{F}^{m} z \in-E^{+}$. Como $\varphi_{2}$ é uma órbita do tipo considerada no primeiro caso, temos $\left\|D_{\mathcal{F} k} \mathcal{F}^{m-k} v\right\| \geq \gamma_{1}\|v\|$ para $v \in C_{i}\left(\mathcal{F}^{k} z\right), i \in\{1,2\}$. Agora, $\varphi_{1}$ é um pedaço de órbita como em (4.4). Logo pelo Corolário 5 existe $\beta_{5}^{\prime}>0$ independente de $\varphi_{1}$ tal que $\left\|D_{z} \mathcal{F}^{k} v\right\| \geq \beta_{5}^{\prime}\|v\|$ para $v \in C(z)$. Assim, observamos que

$$
\left\|D_{z} \mathcal{F}^{m} v\right\|=\left\|D_{\mathcal{F}^{k}} \mathcal{F}^{m-k}\left(D_{z} \mathcal{F}^{k} v\right)\right\| \geq \gamma_{1}\left\|D_{z} \mathcal{F}^{k} v\right\| \geq \gamma_{1} \beta_{5}^{\prime}\|v\|
$$

e assim (4.4) é satisfeita com constante $\beta^{\prime}=\gamma_{1} \beta_{5}^{\prime}$.

\subsubsection{Conclusão da prova de L4.}

Como a aplicação do bilhar $\mathcal{F}$ é tempo-reversível, pode-se mostrar que a parte estável de L4 para $x$ é equivalente à parte instável de $\mathrm{L} 4$ mas com $O$ substituído por $-O$. Também, por hiperbolicidade de $\mathcal{F}$, Teorema 3, o conjunto invariante $\Lambda \subset \mathcal{M}$ onde $\mathcal{F}$ admite uma variedade estável local e uma instável, tem medida total. Para a definição dessas variedades, veja Proposição 5. A variedade estável local (resp. variedade instável local) de $y \in \Lambda$ é denotada por $V_{y}^{s}$ (resp. $V_{y}^{u}$ ). Tendo em vista do tempo de reversibilidade de $\mathcal{F}$, temos $-V_{y}^{s}=V_{-y}^{u}$ e $-V_{y}^{u}=V_{-y}^{s}$ para cada $y \in \Lambda$. Portanto $\Lambda=-\Lambda$. Dessas considerações, segue que a parte instável de L4 com $O$ substituída por $E \cup-E$, e $\Lambda_{x}$ substituído por $\Lambda$ implica $L 4$ (partes estável e instável).

Precisaremos também do seguinte lema técnico. Sua prova pode ser encontrada em [MM13a], Lema 3.6. Para a definição dos conjuntos $W_{y}^{s}$ e $W_{y}^{u}$ veja Definição 11.

Lema 20. $O$ conjunto $\Lambda$ pode ser escolhido tal que satisfaz a seguinte propriedade: se $y \in \Lambda \cap E e$ $z \in W_{y}^{u}$ (resp. $z \in W_{y}^{s}$ ), então $T_{z} W_{y}^{u} \subset C(y)$ (resp. $\left.T_{z} W_{y}^{s} \subset C^{\prime}(y)\right)$.

Proposição 13. Cada ponto $x \in \mathcal{H}$ satisfaz a Condição L4.

Demonstração. Suponhamos que $x \in \mathcal{H}$, e seja $(l, N, O, K)$ a quádrupla associada à $x$ (especificada na Definição 7). Seja $\epsilon_{0}$ como no Lema 10. Iremos provar a parte instável de L4 com $O$ substituída por $E \cup-E, \Lambda_{x}$ substiuído por $\Lambda$ e $\epsilon=\epsilon_{0}$ dado. Como explicado no início dessa subseção, isso implica L4.

Suponhamos que $y \in \Lambda \cap(E \cup-E), z \in(E \cup-E) \cap W_{y}^{u} \cap \mathcal{F}^{k} \mathcal{S}_{1}^{-}\left(\epsilon_{0}\right)$ para algum $k>0$. Observe que $(O, K)$ é igual a $\left(U_{y}, C(y)\right)$ para $y \in E$. Estudaremos os casos $\mathcal{F}^{-k} z \in E$ e $\mathcal{F}^{-k} \notin E$.

Primeiro supomos que $\mathcal{F}^{-k} z \in E$, e considere $v \in T_{z} W_{y}^{u}$. Pelo Lema 20 e a invariância do campo de cone $\left\{\left(U_{y}, C(y)\right)\right\}_{y \in E}$ temos $D_{z} \mathcal{F}^{-k} v \in C\left(\mathcal{F}^{-k} z\right)$ e pela Proposição 12, isso implica, que $\left\|D_{z} \mathcal{F}^{-k} v\right\| \leq 1 / \beta^{\prime}$, onde $\beta^{\prime}$ é a constante que aparece em (4.4). 
Agora, se $\mathcal{F}^{-k} z \notin E$, então há duas possibilidades: ou $\mathcal{F}^{-k} z \in \mathcal{M}^{0} \cap \mathcal{S}_{1}^{-}\left(\epsilon_{0}\right)$ ou $\mathcal{F}^{-k} \in \mathcal{M}^{+} \backslash E$ (uma vez que $\mathcal{M}^{-} \subset E$ ). Estudemos as duas possibilidades separadamente. Primeiro defina $m_{\mp}=$ $\inf \left\{i>0: \mathcal{F}^{-k \mp i} z \in E\right\}$.

Se $\mathcal{F}^{-k} z \in \mathcal{M}^{0} \cap \mathcal{S}_{1}^{-}\left(\epsilon_{0}\right)$, então dado $v \in T_{z} W_{y}^{u}$ e novamente considerando o Lema 20 e a invariância do campo de cone $\left\{\left(U_{y}, C(y)\right)\right\}_{y \in E}$, a Proposição 11 aplicada à $\mathcal{F}^{-k-m_{-}} z$ com $m=$ $m_{-}+m_{+}$e $j=m_{-}$, nos dá $\left\|D_{\mathcal{F}^{-k+m_{+}}} \mathcal{F}^{-m_{+}} v\right\| \leq 1 / \gamma_{2}$. Vemos também que, $\left\|D_{z} \mathcal{F}^{-k-m_{+}} v\right\|$ é igual a 1 se $m_{+}=k$, e não maior que $1 / \beta^{\prime}$ se $m_{+}<k$ pela Proposição 12 . Logo, combinando as desigualdades anteriores obtemos $\left\|D_{z} \mathcal{F}^{-k} v\right\| \leq 1 /\left(\gamma_{2} \min \left\{1, \beta^{\prime}\right\}\right)$, para $v \in T_{z} W_{y}^{u}$.

Se $\mathcal{F}^{-k} z \in \mathcal{M}^{+} \backslash E$, seja $n=n\left(\mathcal{F}^{-k} z\right)$. Quebrando a sequência de números consecutivos $\{-k, \ldots, 0\}$ em três sequências de números consecutivos $\{-k, \ldots,-k+n\}$ e $\left\{-k+n, \ldots,-k+m_{+}\right\}$ e $\left\{-k+m_{+}, \ldots, 0\right\}$, vemos que $D_{z} \mathcal{F}^{k}=D_{\mathcal{F}^{-k+n} z} \mathcal{F}^{-n} \circ D_{\mathcal{F}^{-k+m_{+}}} \mathcal{F}^{-m_{+}+n_{\circ}} D_{z} \mathcal{F}^{-k+m_{+}}$. Logo, dado $v \in T_{z} W_{y}^{u}$, como antes, temos $\left\|D_{z} \mathcal{F}^{-k+m_{+}} v\right\| \leq 1 / \beta^{\prime}$ pela Proposição $12,\left\|D_{\mathcal{F}^{-k+m_{+}}} \mathcal{F}^{-m_{+}+n} v\right\| \leq$ $1 / \delta_{1}$ pelo Lema 15 , e $\left\|D_{\mathcal{F}^{-k+n} z} \mathcal{F}^{-n} v\right\| \leq 1 / \gamma_{1}$ pela Proposição 10 . Combinando as desigualdades anteriores, segue que $\left\|D_{z} \mathcal{F}^{-k} v\right\| \leq 1 /\left(\beta^{\prime} \delta_{1} \gamma_{1}\right)$.

Para a prova completa, observamos que os limites superiores de $\left\|D_{z} \mathcal{F}^{-k} v\right\|$ para os diferentes casos estudados acima não dependem nos dados $y, z, k$ da Condição L4. 

ERGODICIDADE LOCAL. 


\section{Apêndice A}

\section{Teorema da Decomposição Espectral}

Relembramos aqui o Teorema da Decomposição Espectral. Tal Teorema se aplica à uma grande classe de sistemas hiperbólicos, porém aqui iremos formular somente para bilhares. Para sua prova, veja referência [KS86], Teorema 13.1, Parte II.

Teorema 5. Suponha que a aplicação do bilhar $\mathcal{F}$ possui expoentes de Lyapunov não nulos quase sempre em $\mathcal{M}$. Então existe um número enumerável de subconjuntos mensuráveis $E_{0}, E_{1}, \ldots$ de $\mathcal{M}$ dois a dois disjuntos, tais que

1. $\mathcal{M}=\bigcup_{i=0}^{\infty} E_{i}$,

2. $\mu\left(E_{0}\right)=0$, e $\mu\left(E_{i}\right)>0$ para $i \in \mathbb{N}$,

3. $\mathcal{F}\left(E_{i}\right)=E_{i}, e\left(\left.\mathcal{F}\right|_{E_{i}},\left.\mu\right|_{E_{i}}\right)$ é ergódico para $i \in \mathbb{N}$,

4. para cada $i \in \mathbb{N}$, existe $m_{i} \in \mathbb{N}$ subconjuntos mensuráveis dois a dois disjuntos $B_{i, 1}, \ldots, B_{i, m_{i}}, B_{i, m_{i}+1}=$ $B_{i, 1}$ de $\mathcal{M}$ tais que $E_{i}=\bigcup_{j=1}^{m_{i}} B_{i, j}, \mathcal{F}\left(B_{i, j}\right)=B_{i, j+1}$ e $\left(\left.\mathcal{F}^{m_{i}}\right|_{B_{i, j}},\left.\mu\right|_{B_{i, j}}\right)$ é Bernoulli para cada $j=1, \ldots, m_{i}$.

Os conjuntos $E_{i}$ e $B_{i, j}$ são chamados de componente ergódica de $\mathcal{F}$ e componente Bernoulli de $\mathcal{F}$, respectivamente. Esses conjuntos são unicamente definidos, a menos de conjunto de medida nula. 
APÊNDICE A 


\section{Referências Bibliográficas}

[BKM78] C. Boldrighini, M. Keane e F. Marchetti. Billiards in polygons. Ann. Probab., 6(4):532540, 1978. 13

[BL08] L. Bussolari e M. Lenci. Hyperbolic billiards with nearly flat focusing boundaries, I. Phys. D, 237(18):2272-2281, 2008. xiii, 1, 2, 5, 11, 13, 14, 15, 18, 25, 27, 28, 31, 38

[BM99] L. A. Bunimovich e G. Del Magno. Track billiards. Commun. Math. Phys., 288(2):699713, 1999. 2

[Bun74] L. A. Bunimovich. Billiards that are close to scattering billiards. Mat. Sb. (N.S.), 94(136):48-73, 1974. 1

[Bun79] L. A. Bunimovich. On the ergodic properties of nowhere dispersing billiards. Commun. Math. Phys., 65(3):295-312, 1979. 1

[Bun90a] L. A. Bunimovich. A theorem on ergodicity of two-dimensional hyperbolic billiards. Commun. Math. Phys., 130(3):599-621, 1990. 2

[Bun90b] L. A. Bunimovich. On absolutely focusing mirrors. Lect. Notes in Math. Springer, Berlin, 1990. 1

[CH96] N. Chernov e C. Haskell. Nonuniformly hyperbolic K-systems are Bernoulli. Ergodic Theory Dynam. Systems, 16(1):19-44, 1996. 2

[CM06] N. Chernov e R. Markarian. Chaotic Billiards. Mathematical Surveys and Monographs. American Mathematical Society, Providence, RI, 2006. 1, 5, 9, 10, 14, 17, 18, 24, 33

[CT98] N. Chernov e S. Troubetzkoy. Ergodicity of billiards in polygons with pockets. Nonlinearity, 11(4):1095-1102, 1998. 2

[Don91] V. J. Donnay. Using integrability to produce chaos: billiards with positive entropy. Commun. Math. Phys., 141(2):225-257, 1991. 1, 2

[GO74] G. Gallavotti e D. S. Ornstein. Billiards and Bernoulli schemes. Commun. Math. Phys., 38:83-101, 1974. 1

[Hop39] E. Hopf. Statistik der geodätischen Linien in Mannigfaltigkeiten negativer Krümmung. Ber. Verh. Sächs. Akad. Wiss., 91:261-304, 1939. 2, 17

[KS86] A. Katok e J.-M. Strelcyn. Invariant manifolds, entropy and billiards; smooth maps with singularities. Lect. Notes in Math. Springer, Berlin, 1986. 7, 24, 45

[KSS90] A. Krámli, N. Simányi e D. Szász. A transversal fundamental theorem for semi-dispersing billiards. (see also Erratum) Commun. Math. Phys., 129:535-560, 1990. 2

[LW86] C. Liverani e M. P. Wojtkowski. Ergodicity in Hamiltonian systems, (in Dynamics reported). Dynam. Report. Expositions Dynam. Systems (N.S.). Springer, Berlin, 1986. 2, $3,7,10,32$ 
[Mag01] G. Del Magno. Ergodicity of a class of truncated elliptical billiards. Nonlinearity, 14(6):761-1786, 2001. 2

[Mar88] R. Markarian. Billiards with Pesin region of measure one. Commun. Math. Phys., 118(1):87-97, 1988. 1, 2, 24

[Mar93] R. Markarian. New ergodic billiards: exact results. Nonlinearity, 6(5):819-841, 1993. 2

[MM03] G. Del Magno e R. Markarian. Bernoulli elliptical stadia. Commun. Math. Phys., 233(2):7211-230, 2003. 2

[MM13a] G. Del Magno e R. Markarian. A local ergodic theorem for non-uniformly hyperbolic symplectic maps with singularities. Ergodic Theory Dynam. Systems, 33:83-107, 2013. 2, $19,24,42$

[MM13b] G. Del Magno e R. Markarian. Singular sets of hyperbolic planar billiards are regular. Regul. Chaotic Dyn., 18(4):425-452, 2013. 19, 20, 21, 22, 33

[MM14] G. Del Magno e R. Markarian. On the Bernoulli property of planar hyperbolic billiards. Pré-publicação, http://premat.fing.edu.uy/papers/2014/1777.pdf, 2014. 2, 7, 18, 34, 36

[SC87] Ya. G. Sinai e N. I. Chernov. Ergodic properties of some systems of two-dimensional disks and three-dimensional balls. Uspehi Mat. Nauk., 42(3):153-174, 1987. 2

[Sin70] Ya. G. Sinai. Dynamical systems with elastic reflections. Ergodic properties of dispersing billiards. Uspehi Mat. Nauk., 25(2):141-192, 1970. 1, 2

[Szá92] D. Szász. On the K-property of some planar hyperbolic billiards. Commun. Math. Phys., 145(3):595-604, 1992. 2

[Woj85] M. P. Wojtkowski. Invariant families of cones and Lyapunov exponents. Ergodic Theory Dynam. Systems, 5(1):145-161, 1985. 8, 24

[Woj86] M. P. Wojtkowski. Principles for the design of billiards with nonvanishing Lyapunov exponents. Comm. Math. Phys., 105(3):391-414, 1986. 1, 7, 8, 9, 25, 32 